\title{
Development and Application of Genic Simple Sequence Repeat Markers from the Transcriptome of Loquat
}

\author{
Xiaoying Li and Hongxia Xu \\ Institute of Horticulture, Zhejiang Academy of Agricultural Sciences, Shiqio road 139, Hangzhou, \\ P.R. China, 310021 \\ Jianjun Feng \\ Ninghai Agriculture \& Forestry Bureau, Ningbo, Zhejiang, P.R. China, 315600 \\ Junwei Chen ${ }^{1}$ \\ Institute of Horticulture, Zhejiang Academy of Agricultural Sciences, Shiqio road 139, Hangzhou, \\ P.R. China, 310021
}

\begin{abstract}
Additional index words. cluster, genetic analysis, microsatellite, species, transferability
ABstract. Deep transcriptome sequencing allows for the acquisition of large-scale microsatellite information, and it is especially useful for genetic diversity analysis and mapping in plants without reference genome sequences. In this study, a total of 14,004 simple sequence repeats (SSRs) were mined from 10,511 unigenes screening of 63,608 nonredundant transcriptome unigenes in loquat (Eriobotrya japonica) with a frequency of 22 SSR loci distributed over 100 unigenes. Dinucleotide and trinucleotide repeat SSRs were dominant, accounting for $20.62 \%$, and $42.1 \%$ of the total, respectively. Seventy primer pairs were designed from partial SSRs and used for polymerase chain reaction (PCR) amplification. Of these primer pairs, 54 exhibited amplification and 33 were polymorphic. The number of alleles at these loci ranged from two to 17, and the polymorphism information content values ranged from 0.24 to 0.89 . We tested the transferability of 33 SSR polymorphic primer pairs in apple and pear, and the transferability rates in these two species were $90.9 \%$ and $87.9 \%$, respectively. A high level of marker polymorphism was observed in apple [Malus $\times$ domestica $(66.7 \%)]$, whereas a low level was observed in pear [Pyrus $\mathbf{s p .}(51.5 \%)]$. In addition, the PCR products from seven SSR primer pairs were selected for sequence analysis, and $89.2 \%$ of the fragments were found to contain SSRs. SSR motifs were conserved among loquat, apple, and pear. According to our sequencing results for real SSR loci, $\approx 12,490$ SSR loci were present in these loquat unigenes. The cluster dendrogram showed a distinct separation into different groups for these three species, indicating that these SSR markers were useful in the evaluation of genetic relationships and diversity between and within the species of Maloideae in the Rosaceae. The results of our identified SSRs should be useful for genetic linkage map construction, quantitative trait locus mapping, and molecular marker-assisted breeding of loquat and related species.
\end{abstract}

Loquat belongs to the family Rosaceae and is an important evergreen fruit crop native to south-central China (Gisbert et al., 2009a, 2009b). It has become popular with consumers because of its sweet and edible fruit (Lin et al., 2004), which can be sorted into red- and white-fleshed cultivars (Fu et al., 2012). Over 300 cultivars have been recorded and preserved, most of which are landraces with high genetic diversity (Qiu and Zhang, 1996). To more efficiently use these abundant loquat germplasm resources in genetic breeding, they must be evaluated and identified using efficient molecular methods.

Microsatellites or SSR are 1- to 6-bp DNA regions repeated in tandem that are ubiquitous in both prokaryotes and eukaryotes (Gao et al., 2013). As a result, SSRs are simple and efficient markers useful in the analysis of codominance and highly polymorphic lines. SSRs have been used extensively in genetic

Received for publication 28 Mar. 2014. Accepted for publication 25 Apr. 2014. We appreciate our funding from the China Postdoctoral Science Foundation (2013M531482); the Key Project for New Agricultural Cultivar Breeding in Zhejiang Province, China (2012C12904-5); the National Science Foundation for Distinguished Young Scholars of China (Grant No. 31101530); the Science and Technology Plan projects of Ningbo (2013C11022); and Science and Technology Innovation and Promotion Project (2014CX015).

${ }^{1}$ Corresponding author. E-mail: chenjunwei@mail.zaas.ac.cn. diversity assessments (Li et al., 2010a), cultivar fingerprinting (Wang et al., 2011), marker-assisted breeding (Gutiérrez et al., 2011), and diversity and linkage map construction studies (Chen et al., 2006; Pazos-Navarro et al., 2011). As a result of the lack of genomic information for most non-model plant species, it is expensive and labor-intensive to develop traditional SSRs from genomic DNA library screening (Temnykh et al., 2001). With the development of next-generation sequencing in transcriptomics, creating transcriptome-level sequence collections has become much more cost-effective. A wealth of genic SSRs, single nucleotide polymorphism, and other genetic markers have been identified and developed for non-model plant species (Li et al., 2013c, 2013d; Mardis, 2008; Zhang et al., 2012a). In comparison with genomic SSR markers, genic SSR markers are based on expressed sequences from coding regions and are potentially tightly linked with functional genes that control important phenotypic characteristics (Ramchiary et al., 2011). Genic SSRs derived from transcriptome or expressed sequence tag (EST) sequences have been widely mined and applied in molecular biology studies for numerous fruit tree species such as pomegranate [Punica granatum (Ono et al., 2011)], litchi [Litchi chinensis (Li et al., 2013a)], highbush blueberry [Vaccinium corymbosum 
(Rowland et al., 2012)], peach [Prunus persica (Wang et al., 2013), pear [Pyrus pyrifolia (Yue et al., 2014)], and Nules clementine [Citrus clementina (Luro et al., 2008)], but they have not been developed from the loquat transcriptome.

Genic SSRs can also be developed from the data for ESTs (Bouck and Vision, 2006). However, only four loquat ESTs have been submitted to GenBank (as of 1 Jan. 2014), indicating a lack of development of EST-SSR markers in the species. Most of the EST-SSRs used in loquat genetic diversity studies have been derived from apple ( $\mathrm{Li}$ et al., 2013b). To address this knowledge gap, we report the first development of genic SSR markers based on loquat transcriptome sequences obtained in our laboratory, which provides a valuable resource to complement SSR collections. The detailed information on the transcriptome will be described elsewhere. The aim of this study was to explore the SSR loci of transcriptome sequences in loquat, analyze their distribution regularity, and design primers to test the authenticity and identify the transferability of these SSR loci in other Maloideae species of the Rosaceae. These results will prove very useful for the evaluation of genetic diversity, cultivar identification, and marker-assisted selection in future research.

\section{Materials and Methods}

Plant Materials. A total of 33 accessions were used for the development and transferability testing of genic SSR markers in this study. Young leaf samples of 13 loquats and 10 pear cultivars (Table 1) were collected from the Loquat and Pear Germplasm Resources Garden (Yangdu Agricultural Research Station, Institute of Horticulture, Zhejiang Academy of Agricultural Sciences, Hangzhou, P.R. China). Young leaf samples of 10 apple cultivars were collected from the National Germplasm Resources Garden of Apple (Zhengzhou Academy of Agricultural Sciences, Zhengzhou, P.R. China). The names of the cultivars are listed in Table 1 .

DNA isolation. Genomic DNA for each cultivar was isolated from young leaves using the DNeasy Plant Mini Kit (Qiagen, Valencia, CA) according to the manufacturer's recommendations. DNA concentrations were quantified using a spectrophotometer (U-0080D; Hitachi High-Technologies
Corp., Tokyo, Japan), and subsamples were run on $1 \%$ agarose gels to test for sample integrity (data not shown). All samples were stored at $-80^{\circ} \mathrm{C}$ until further use.

Mining of SSR baSEd ON THE TRANSCRIPTOME DATA SET. A total of 63,608 unigenes were obtained from the 'Ninghaibai' loquat fruit transcriptome acquired in our laboratory (unpublished data). The genic SSR loci in the loquat fruit transcriptome were searched from the unigenes using MISA (Thiel et al., 2003), a Perl script able to detect both perfect and compound microsatellites in nucleotide sequences. Compound microsatellites were defined as repeats interrupted by a nonrepetitive sequence of a maximum 50 nucleotides. MISA was set with the following minimum length criteria for the extraction of repeated units (unit size/minimum number of repeats): at least six dinucleotides $(2 / 6)$, at least five trinucleotides $(3 / 5)$, three tetranucleotides $(4 / 3)$, three pentanucleotides $(5 / 3)$, and three hexanucleotides $(6 / 3)$. To reduce the errors caused by the parameter set, we reduced the necessary repetitions of the dinucleotide through pentanucleotide motifs, according to previous work conducted by Jung et al. (2005) and Poncet et al. (2006). Sequences containing corresponding repeat units were selected for marker development.

SSR PRIMER DESIGN. Primers for genic SSRs in the microsatellite sequences were designed using Primer3 (Rozen and Skaletsky, 2000) based on the following core criteria: a guaninecytosine (GC) content between $40 \%$ and $60 \%$ with an optimum value of 50\%; an annealing temperature between 54 and $63{ }^{\circ} \mathrm{C}$; a minimum product length of $100 \mathrm{bp}$; and a primer length of 18 to 24 nucleotides. Product sizes ranged between 150 and $350 \mathrm{bp}$. Primers were synthesized by Shanghai Jierui Co. (Shanghai, P.R. China). The sequences for SSR primer design were shown in supplementary file.

PCR AMPLifiCATION AND GEL ELECTROPHORESIS. PCR amplification reactions were performed using a Biometra Authorized Thermal Cycler (An Analytik Jena Co., Thuringia, Germany) and conducted in a total volume of $30 \mu \mathrm{L}$ containing 30 ng template DNA, $30 \mu$ mol each primer, $15 \mu \mathrm{L} 2 \times$ Taq mix, and double-distilled (dd) $\mathrm{H}_{2} \mathrm{O}$ to reach the total volume. PCR conditions were as follows: $94{ }^{\circ} \mathrm{C}$ for $5 \mathrm{~min}$ followed by 40 cycles of $94{ }^{\circ} \mathrm{C}$ for $30 \mathrm{~s}, 50{ }^{\circ} \mathrm{C}$ to $60{ }^{\circ} \mathrm{C}$ for $40 \mathrm{~s}$, and $72{ }^{\circ} \mathrm{C}$ for

Table 1. Cultivars used for genic simple sequence repeat marker amplification and transferability in this study.

\begin{tabular}{|c|c|c|c|c|c|}
\hline Code & Cultivar name & Genus and/or species & Code & Cultivar name & Genus and/or species \\
\hline 1 & Ninghaibai & Eriobotrya japonica & 18 & Cuiguan & Pyrus \\
\hline 2 & Oohusa & E. japonica & 19 & Laiyangli & Pyrus \\
\hline 3 & Jiefangzhong & E. japonica & 20 & Sucui 1 & Pyrus \\
\hline 5 & Guanyu & E. japonica & 22 & Huanghuali & Pyrus \\
\hline 6 & Marc & E. japonica & 23 & Xiuyu & Pyrus \\
\hline 7 & Gangbeihuangrou & E. japonica & 24 & Huashuai & Malus $\times$ domestica \\
\hline 9 & Changhong 3 & E. japonica & 26 & Songbenjin & M. $\times$ domestica \\
\hline 10 & Maomu & E. japonica & 27 & Enpai & M. $\times$ domestica \\
\hline 11 & Senweizaosheng & E. japonica & 28 & Guoguang & M. $\times$ domestica \\
\hline 12 & Zaozhong 6 & E. japonica & 29 & Maiyan & M. $\times$ domestica \\
\hline 13 & Tianzhong & E. japonica & 30 & Freedom & M. $\times$ domestica \\
\hline 14 & Qingxiang & Pyrus & 31 & Hongjin & M. $\times$ domestica \\
\hline
\end{tabular}


$1 \mathrm{~min}$ and a final extension step at $72^{\circ} \mathrm{C}$ for $10 \mathrm{~min}$. To control the PCR product integrity, a $5-\mu \mathrm{L}$ aliquot was visualized on a $1.5 \%$ agarose gel. The remaining reaction was run on a $6 \%$ polyacrylamide gel according to the specifications outlined in Li et al. (2013b) and then stained with $2 \%$ silver nitrate according to Bassam et al. (1991). The stained gels were photographed in white light.

Cross-SPeCies AMPlification. To assess the transferability of our SSR markers, we tested their amplification in two other Rosaceae species: apple and pear. The PCR amplification reactions were performed like in the previously described protocols.

Fragment RECOVERY AND SEQUENCING. The target fragments obtained from the acrylamide gels were cut, washed twice in $1 \mathrm{~mL} \mathrm{ddH} \mathrm{H}_{2} \mathrm{O}$, and recovered according to Li et al. (2013b). The PCR conditions were performed as described previously, but only single-specific fragments detected on the agarose gels were cut. Further recovery was conducted using the DNA gel extraction kit produced by Shanghai Songon Biotech Co. (Shanghai, China), and sequencing was also conducted by Shanghai Songon Biotech Co.

DAta COLlection AND ANALysis. The following parameters were estimated from the microsatellite marker data: number of alleles per locus: expected heterozygosity $\left[H e=1-\Sigma p i^{2}\right.$, where $p i$ is the frequency of the $i^{\text {th }}$ allele $\left.(\mathrm{Nei}, 1973)\right]$ and observed heterozygosity ( $\mathrm{Ho}$, calculated as the number of heterozygous genotypes divided by the total number of genotypes). According to the presence or absence of bands on the polyacrylamide gel, the same electrophoretic banding patterns were recorded as 1 , whereas other patterns were recorded as 0 . The polymorphism data were entered into Excel (Microsoft Inc., Redmond, WA) for cluster analysis using NYSYS-pc software (Rohlf, 2005), and a dendrogram was generated for the analysis of genetic relationships and diversity among the cultivars of loquat, apple, and pear. SSR loci recovered from the polyacrylamide gels were checked using SSRIT software (da Maia et al., 2008).

\section{Results}

Distribution, FREQUenCy, AND CHARACTERISTICS OF SSR SEQUENCES. In this study, MISA was used to identify SSR markers in the unique sequences. From the 63,608 sequences examined in the present study, representing a total size of 48,295,300 bp, 14,004 SSRs were identified in 10,511 sequences for a frequency of 22 SSR loci distributed over 100 unigenes. The distribution density represents one SSR locus for every $3.45 \mathrm{~kb}(\mathrm{~kb} / \mathrm{SSR})$. A total of 6754 sequences contained only one SSR, whereas 2544 sequences contained more than one SSR locus; 1213 SSR loci were present in compound formation. This result indicated that SSRs were quite abundant in the loquat transcriptome sequences (Table 2).

The composition of SSRs was as follows: $2887(20.62 \%)$ dinucleotides, $5896(42.1 \%)$ trinucleotides, 2161 (15.43\%) tetranucleotides, $622(4.44 \%)$ pentanucleotides, and 850 $(6.07 \%)$ hexanucleotides (Table 3$)$. The most abundant motifs among the dinucleotides and trinucleotides were AG/CT and AAG/CTT with frequencies of $85.9 \%$ and $22.17 \%$, respectively. The highest frequency tetranucleotide motif was AAAG/ CTTT with a frequency of $13.98 \%$. There were 93 kinds of pentanucleotide motif with AAAAG/CTTTT comprising $8.53 \%$. The most predominant hexanucleotide motif was
Table 2. Total number and frequency distribution of each repeat type including mono, di-, tri, tetra-, penta-, and hexanucleotide in loquat.

\begin{tabular}{lrccc}
\hline Repeat type & $\begin{array}{c}\text { SSRs } \\
\text { (no.) }\end{array}$ & $\begin{array}{c}\text { SSRs } \\
(\%)\end{array}$ & $\begin{array}{c}\text { SSR } \\
\text { frequency }(\%)\end{array}$ & $\begin{array}{c}\text { Avg } \\
\text { length (kb) }\end{array}$ \\
\hline Mononucleotide & 1,588 & 11.33 & 2.5 & 30.41 \\
Dinucleotide & 2,887 & 20.62 & 4.53 & 16.73 \\
Trinucleotide & 5,896 & 42.1 & 9.26 & 8.19 \\
Tetranucleotide & 2,161 & 15.43 & 3.4 & 22.35 \\
Pentanucleotide & 622 & 4.44 & 0.98 & 77.65 \\
Hexanucleotide & 850 & 6.07 & 1.33 & 56.81 \\
Total SSRs (no.) & 14,004 & 100 & 22.02 & 3.45 \\
\hline
\end{tabular}

$\overline{\mathrm{SSRs}}=$ simple sequence repeats.

Table 3. The number of major SSR motifs of di-, tri, tetra-, penta-, and hexanucleotide and their frequency in loquat.

\begin{tabular}{|c|c|c|c|c|}
\hline Repeat type & SSR motif & $\begin{array}{l}\text { SSRs } \\
\text { (no.) }\end{array}$ & $\begin{array}{c}\text { Total } \\
\text { SSRs (no.) }\end{array}$ & $\begin{array}{c}\text { SSRs } \\
(\%)\end{array}$ \\
\hline \multirow[t]{4}{*}{ Dinucleotide } & $\mathrm{AG} / \mathrm{CT}$ & 2480 & 2887 & 85.9 \\
\hline & $\mathrm{AC} / \mathrm{GT}$ & 304 & & 10.53 \\
\hline & $\mathrm{AT} / \mathrm{AT}$ & 97 & & 3.36 \\
\hline & $\mathrm{CG} / \mathrm{CG}$ & 6 & & 0.21 \\
\hline \multirow[t]{10}{*}{ Trinucleotide } & $\mathrm{AAG} / \mathrm{CTT}$ & 1307 & 5896 & 22.17 \\
\hline & $\mathrm{AGG} / \mathrm{CCT}$ & 1116 & & 18.93 \\
\hline & $\mathrm{ACC} / \mathrm{GGT}$ & 713 & & 12.1 \\
\hline & ACG/CTG & 622 & & 10.55 \\
\hline & AGC/CGT & 621 & & 10.53 \\
\hline & CCG/CGG & 406 & & 6.89 \\
\hline & $\mathrm{AAC} / \mathrm{GTT}$ & 398 & & 6.75 \\
\hline & AGT/ATC & 315 & & 5.34 \\
\hline & ACT/ATG & 298 & & 5.05 \\
\hline & AAT/ATT & 100 & & 1.69 \\
\hline \multirow[t]{5}{*}{ Tetranucleotide } & AAAG/CTTT & 302 & 2161 & 13.98 \\
\hline & AAAT/ATTT & 229 & & 10.59 \\
\hline & AAAC/GTTT & 194 & & 8.98 \\
\hline & AAGG/CCTT & 148 & & 6.85 \\
\hline & Others & 1288 & & 59.6 \\
\hline \multirow[t]{5}{*}{ Pentanucleotide } & AAAAG/CTTTT & 53 & 622 & 8.52 \\
\hline & AAAAT/ATTTT & 52 & & 8.36 \\
\hline & AAAAC/GTTTT & 45 & & 7.23 \\
\hline & AAACC/GGTTT & 22 & & 3.54 \\
\hline & Others & 450 & & 72.35 \\
\hline \multirow{5}{*}{ Hexanucleotide } & AGAGGG/CCCTCT & 26 & 850 & 3.06 \\
\hline & AAGAGG/CCTTCT & 23 & & 2.71 \\
\hline & AAAAAG/CTTTTT & 20 & & 2.35 \\
\hline & ACCTCC/AGGTGG & 20 & & 2.35 \\
\hline & Others & 761 & & 89.53 \\
\hline
\end{tabular}

$\overline{\mathrm{SSR}}=$ simple sequence repeat.

\section{AGAGGG/CCCTCT (3.06\%) followed by AAGAGG/} CCTTCT $(2.71 \%)$

LENGTH DISTRIBUTION AND LOCATION OF SSR Motifs. The length of the microsatellite sequences ranged from 10 to 25 bases. Significant variation occurred in the length of the sequences, which deviated from the normal distribution. The most frequent microsatellite repeat length range was between 10 and 15 bp (Fig. 1), whereas the second most frequent range was between 13 and $16 \mathrm{bp}$. Only nine $(0.06 \%)$ of the sequences were longer than $30 \mathrm{bp}$. A negative correlation existed between abundance, variation, rate and microsatellite length (Fig. 1). 

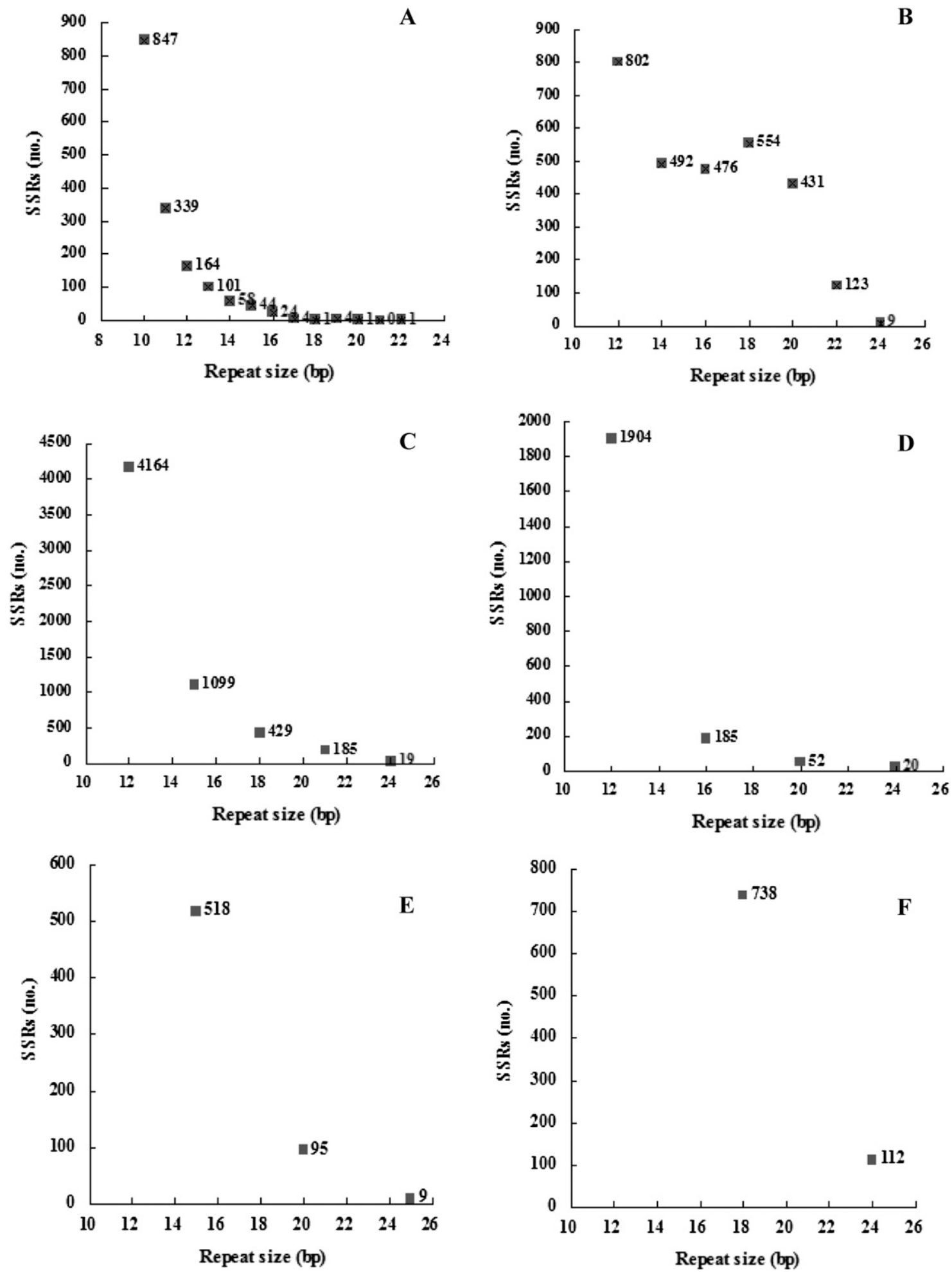

Fig. 1. Frequency distribution of simple sequence repeat (SSR) motif repeat size (loci length) in loquat. The $x$-axis indicates the length of SSRs motif. The y-axis indicates the number of SSRs with different lengths: (A) nucleotide repeats of different lengths, (B) dinucleotide repeats of different lengths, (C) trinucleotide repeats of different lengths, (D) tetranucleotide repeats of different lengths, (E) pentanucleotide repeats of different lengths, and $(\mathbf{F})$ hexanucleotide repeats of different lengths.

Moreover, most dinucleotide and hexanucleotide motifs were found in noncoding regions, whereas trinucleotide, tetranucleotide, and pentanucleotide motifs occurred more frequently in the coding regions.
IDENTIFICATION AND POLYMORPHISM ANALYSIS OF SSR LOCI. Of the 70 SSR primer pairs, 54 (77.1\%) generated clear DNA banding patterns with the expected size, and 33 SSR primers amplified polymorphic PCR products (Table 4). Figure 2 shows 


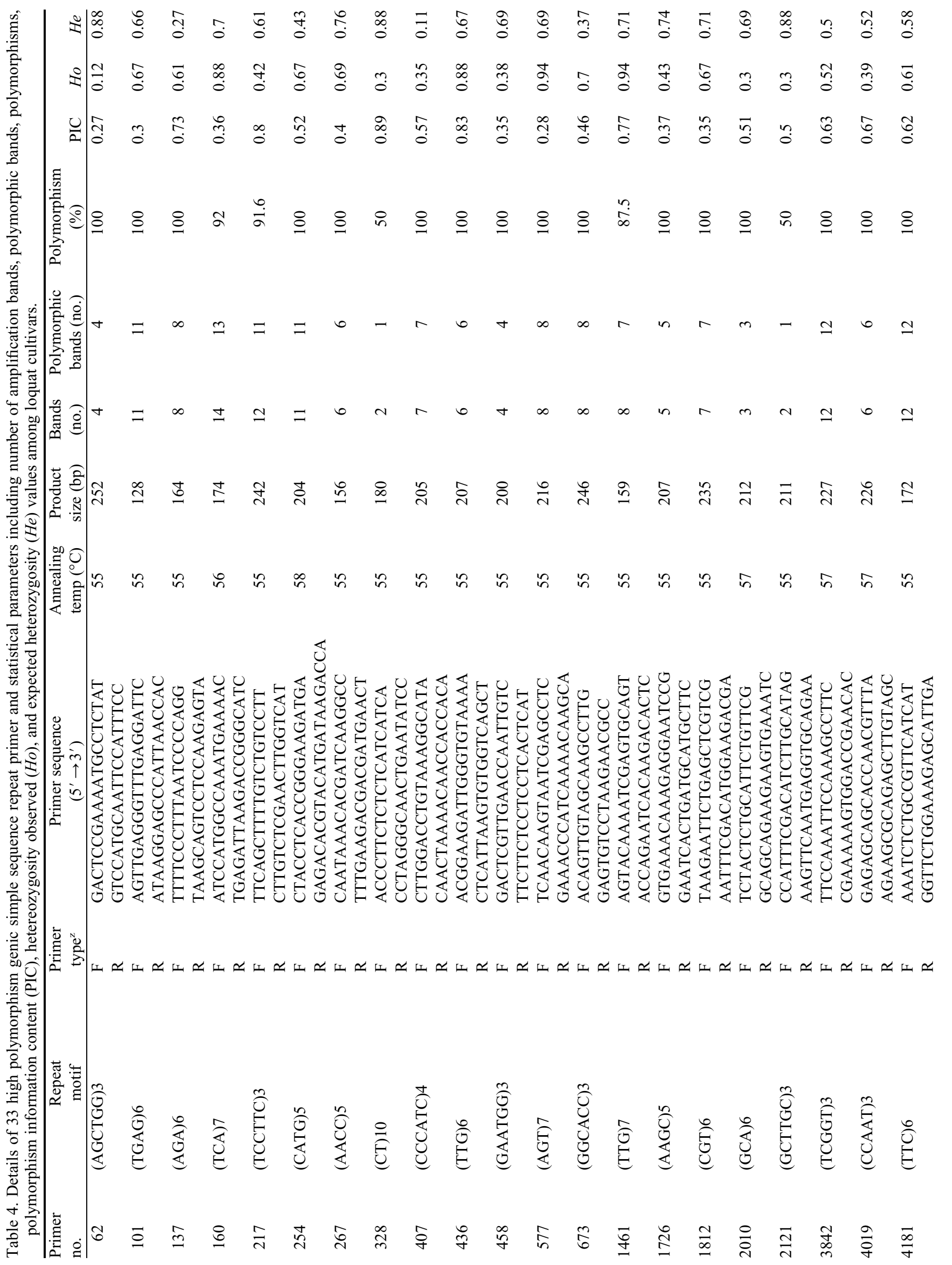




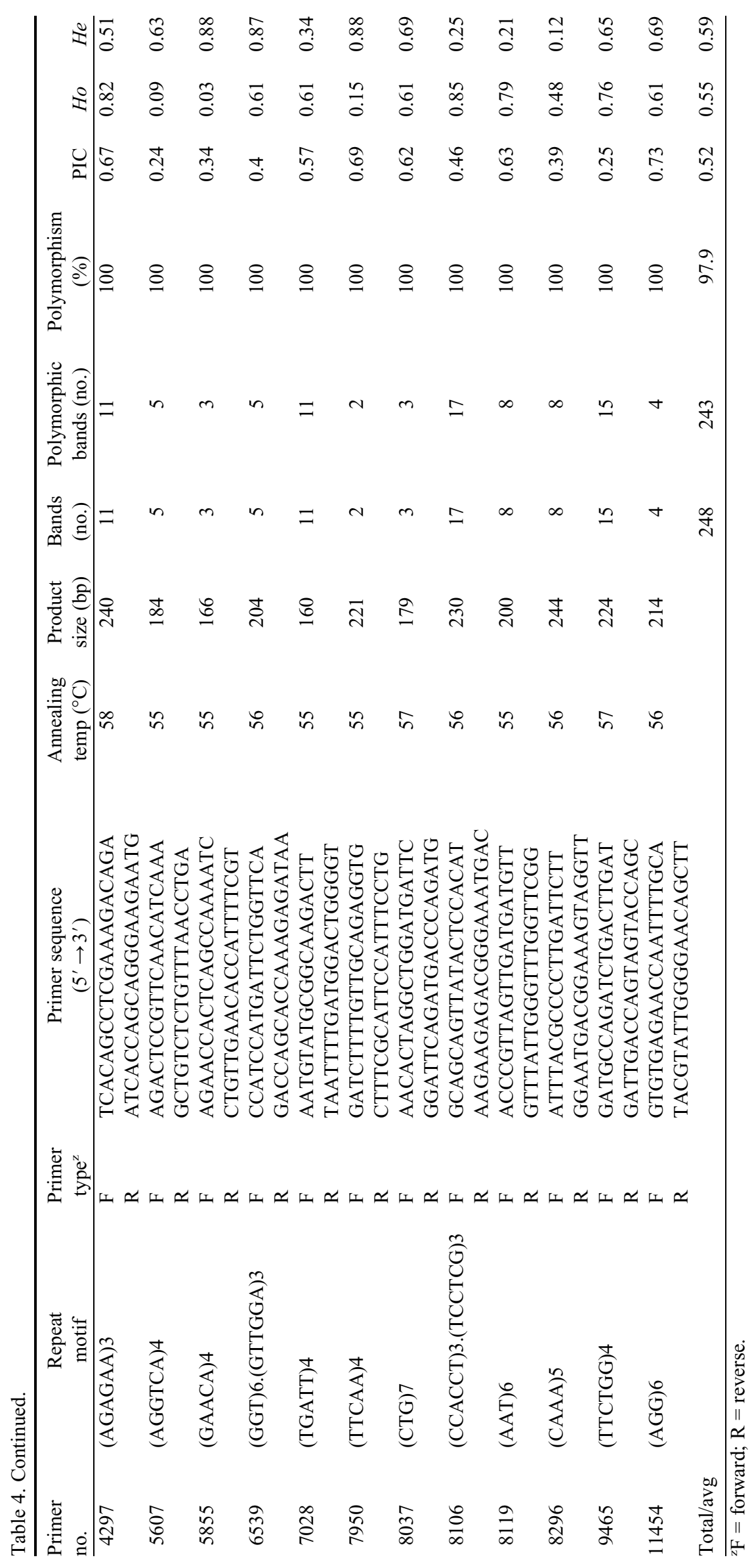




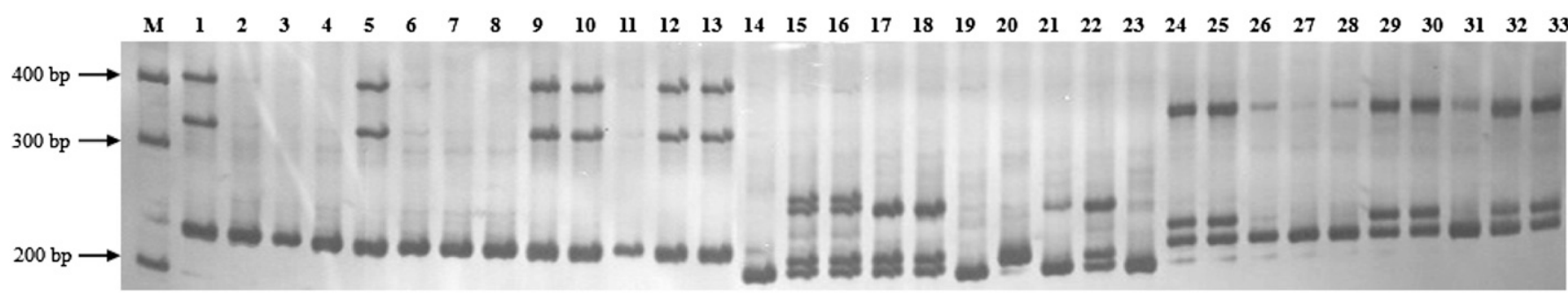

A

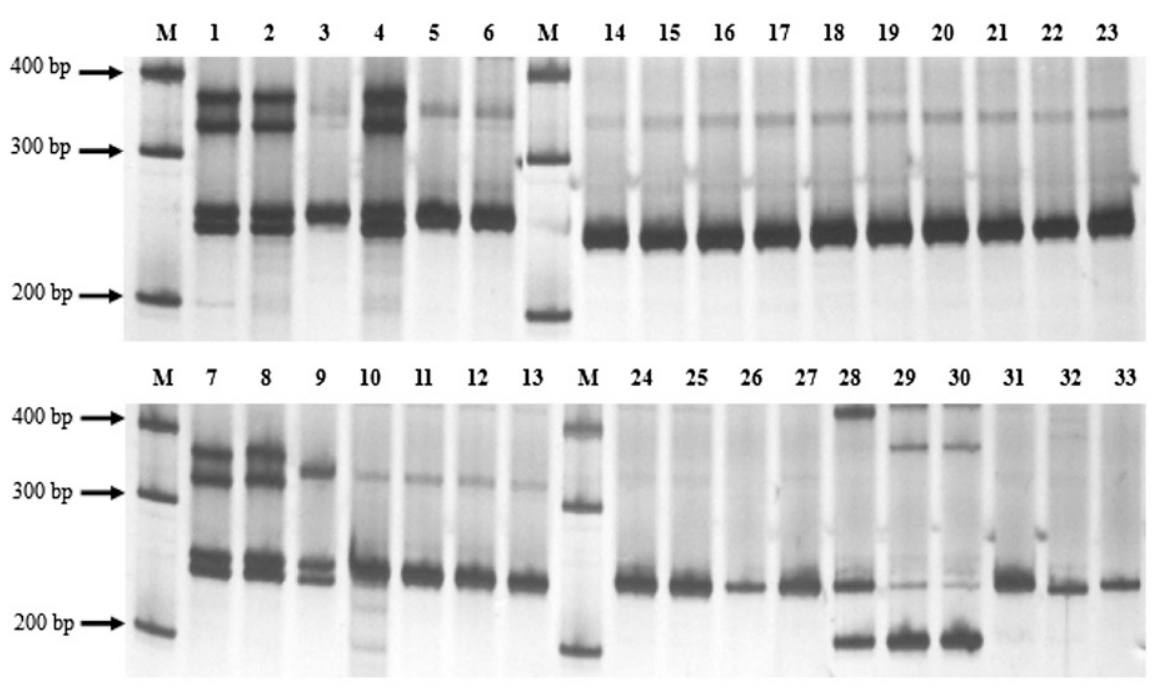

B

Fig. 2. Representative polyacrylamide gel images of genic simple sequence repeat (SSR) marker amplified by primer pairs 4297 and $8296:$ Maker DL2000 (M), accession numbers of loquat (one to 13), accession numbers of pear (14 to 23), accession numbers of apple (24 to 33). Cultivar names are listed in Table 1 . (A) Amplified result of primer 4297; (B) amplified result of primer 8296.

representative polyacrylamide gels amplified with primer pairs 4297 and 8296, respectively. A total of 248 alleles were obtained from the accessions, representing an average of 7.5 alleles per SSR marker and $243(97.9 \%)$ of them were polymorphic bands. The number of alleles obtained for each primer ranged between two and 17. The polymorphic information content ranged from 0.24 to 0.89 with an average of 0.52 , indicating that the SSRs developed in this study had high discriminatory power. The Ho value (heterozygosity) ranged from 0.03 to 0.94 with an average of 0.55 , and the $H \mathrm{e}$ value (expected heterozygosity) ranged from 0.11 to 0.88 with an average of 0.59 (Table 4).

Transferability OF SSR markers. To test the interspecific transferability of these SSR markers in apple and pear, which belong to the same subfamily (Maloideae) as loquat, PCR amplification was conducted in 10 apple and 10 pear cultivars. Clear DNA fingerprints were obtained from the PCR amplification of apple and pear DNA using the loquat SSR primers as seen in the polyacrylamide gels of the SSR bands amplified by the primer pairs 4297 and 8296 (Fig. 2). Of the primer pairs, 30 produced the anticipated SSR bands in apple and 29 in pear, representing transferability rates of $\approx 90.9 \%$ and $87.9 \%$, respectively. A high level of marker polymorphism was observed in apple $(66.7 \%)$, whereas a low rate was seen in pear $(51.5 \%)$. This result indicated that the SSR primers derived from loquat had a high generality among Maloideae species.
Sequence characteristic of PCR products. Polyacrylamide fragments from seven primer pairs $(254,267,3842$, $4181,5855,7028$, and 8296 ) were selected for further sequence analysis. Analysis of the 43 fragments indicated that $37(88 \%)$ were successfully recovered and sequenced, of which four fragments contained no repeat motif and 33 expressed SSR loci, representing a rate of $89.2 \%$ (Table 5). According to our sequencing results for real SSR loci, $\approx 12,490$ SSR loci are found in the loquat unigenes, but the SSR motifs were only partly conserved between loquat, apple, and pear. The majority of SSR markers developed from transcription sequences in this study contained real SSR loci. More than $50 \%$ of the PCR products obtained from apple and pear had different sizes than were expected. Most of the repeat fragment motifs were different from those of the loquat cultivars. These observed differences may the result of genetic divergence among the species (Table 5). The results also showed that different cultivars can share the same allele: sample Nos. 7, 8, and 9 were amplified from the three loquat cultivars Gangbeihongsha, Changhong 3, and Tianzhong, respectively. Compared with the original sequences, the identity values were more than $90 \%$. Moreover, for the primer pair 5855 , six sequences were recovered from three loquat and three pear cultivars; all cultivars shared the same allele, but the identity was lower in pear. Interestingly, different fragment sizes could be amplified from the same cultivar that contained the same repeating unit. For example, Nos. 22 and 23, the fragments 
Table 5. Characteristics of fragments including amplification primer, expected and actual repeat motif, product size, and homology with original sequence recovered from polyacrylamide gel of different loquat, apple, and pear cultivars.

\begin{tabular}{|c|c|c|c|c|c|c|c|}
\hline Code & $\begin{array}{c}\text { Primer } \\
\text { no. }\end{array}$ & $\begin{array}{c}\text { Repeat motif } \\
\text { in form } \\
\text { sequence }\end{array}$ & $\begin{array}{c}\text { Expected } \\
\text { product } \\
\text { (bp) }\end{array}$ & $\begin{array}{l}\text { Fragment } \\
\text { size (bp) }\end{array}$ & $\begin{array}{c}\text { Fragment } \\
\text { repeat } \\
\text { motif } \\
\end{array}$ & $\begin{array}{c}\text { Homology } \\
\text { with original } \\
\text { sequence }(\%)\end{array}$ & $\begin{array}{l}\text { Original } \\
\text { cultivar }\end{array}$ \\
\hline 1 & 254 & (CATG)5 & 204 & 183 & CATG & 80.88 & Sucui 1 \\
\hline 3 & & & & 205 & CATG & 71.49 & Huanghuali \\
\hline 4 & & & & 213 & (CATG)5 & 79.39 & Huashuai \\
\hline 5 & & & & 174 & (CATG)2 & 79.41 & Enpai \\
\hline 7 & 267 & $(\mathrm{AACC}) 5$ & 156 & 156 & $(\mathrm{AACC}) 5$ & 93.89 & Gangbeihongsha \\
\hline 8 & & & & 147 & $(\mathrm{AACC}) 5$ & 93.95 & Changhong 3 \\
\hline 9 & & & & 145 & $(\mathrm{AACC}) 5$ & 90.38 & Tianzhong \\
\hline 10 & & & & 159 & $(\mathrm{AACC}) 8$ & 83.65 & Qingxiang \\
\hline 11 & & & & 150 & $(\mathrm{AACC}) 3$ & 79.49 & Zaosuhong \\
\hline 12 & & & & 111 & - & 34.62 & Sucui 1 \\
\hline 17 & & & & 255 & $(\mathrm{TCGGT}) 3$ & 90.17 & Songbenjin \\
\hline 18 & 4181 & (TTC)6 & 172 & 181 & $(\mathrm{TTC}) 5$ & 60.5 & Huanghuali \\
\hline 19 & 5855 & (GAACA)4 & 166 & 163 & (GAACA)4 & 94.21 & Jiefangzhong \\
\hline 20 & & & & 160 & (GAACA)4 & 91.87 & Guanyu \\
\hline 21 & & & & 160 & (GAACA)4 & 92.46 & Marc \\
\hline 22 & & & & 160 & (GAACA)4 & 80.7 & Qingxiang \\
\hline 23 & & & & 157 & (GAACA)4 & 78.95 & Qingxiang \\
\hline 24 & & & & 159 & (GAACA)4 & 83.63 & Zaojinsu \\
\hline 25 & 7028 & $($ TGATT) 4 & 160 & 152 & (TGATT)4 & 89.38 & Changhong 3 \\
\hline 26 & & & & 147 & (TGATT)4 & 87.5 & Senweizaosheng \\
\hline 27 & & & & 116 & - & 39.13 & Zaosuhong \\
\hline 35 & & & & 234 & (CAAA) 4 & 84.49 & Songbenjin \\
\hline 36 & & & & 198 & $(\mathrm{CAAA}) 2$ & 74.59 & Guoguang \\
\hline 37 & & & & 198 & $(\mathrm{CAAA}) 2$ & 75.1 & Freedom \\
\hline
\end{tabular}

derived from primer 5855 in the pear cultivar Qingxiang, shared the same locus.

Cluster analysis. In this study, an analysis of the genetic diversity among the 33 cultivars of loquat, apple, and pear was conducted using the polymorphic alleles obtained from the developed SSR primer pairs. A clustering diagram showed that the coefficients ranged from 0.58 to 0.96 , and three major groups were identified at a similarity index of 0.67 (Fig. 3), which was consistent with the presence of three different species. In addition, a greater genetic relationship was observed between apple and loquat than between loquat and pear. In the loquat group, 13 cultivars were clustered into three subgroups with a high similarity coefficient (0.88). The first and the third loquat subgroups contained white-fleshed cultivars, whereas eight red-fleshed cultivars were clustered into the second subgroup. In the apple group, cultivars with the same geographical distribution such as Huashuai and Huaguan were clustered together with a genetic similarity coefficient of 0.92 . In the pear group, all 10 cultivars were clearly separated in Figure 3.

\section{Discussion}

SSR MARKER FREQUENCY AND DISTRIBUTION IN LOQUAT TRANSCRIPTOME. Genetic SSR markers are widely applied in diversity analysis, plant cultivar identification, and the construction of genetic linkage maps in population studies ( $\mathrm{Li}$ et al., 2010b). As sequencing technology has developed, deep transcriptome sequencing has become a good resource for the development of SSRs because of the enormous amount of generated sequence data (Zheng et al., 2013). Markers based on transcriptome sequences are useful for the detection of functional variation and gene-associated genetic analysis. Based on the 63,608 nonredundant unigene sequences used in this study, a total of 12,416 SSRs were identified, excluding mononucleotide repeat 


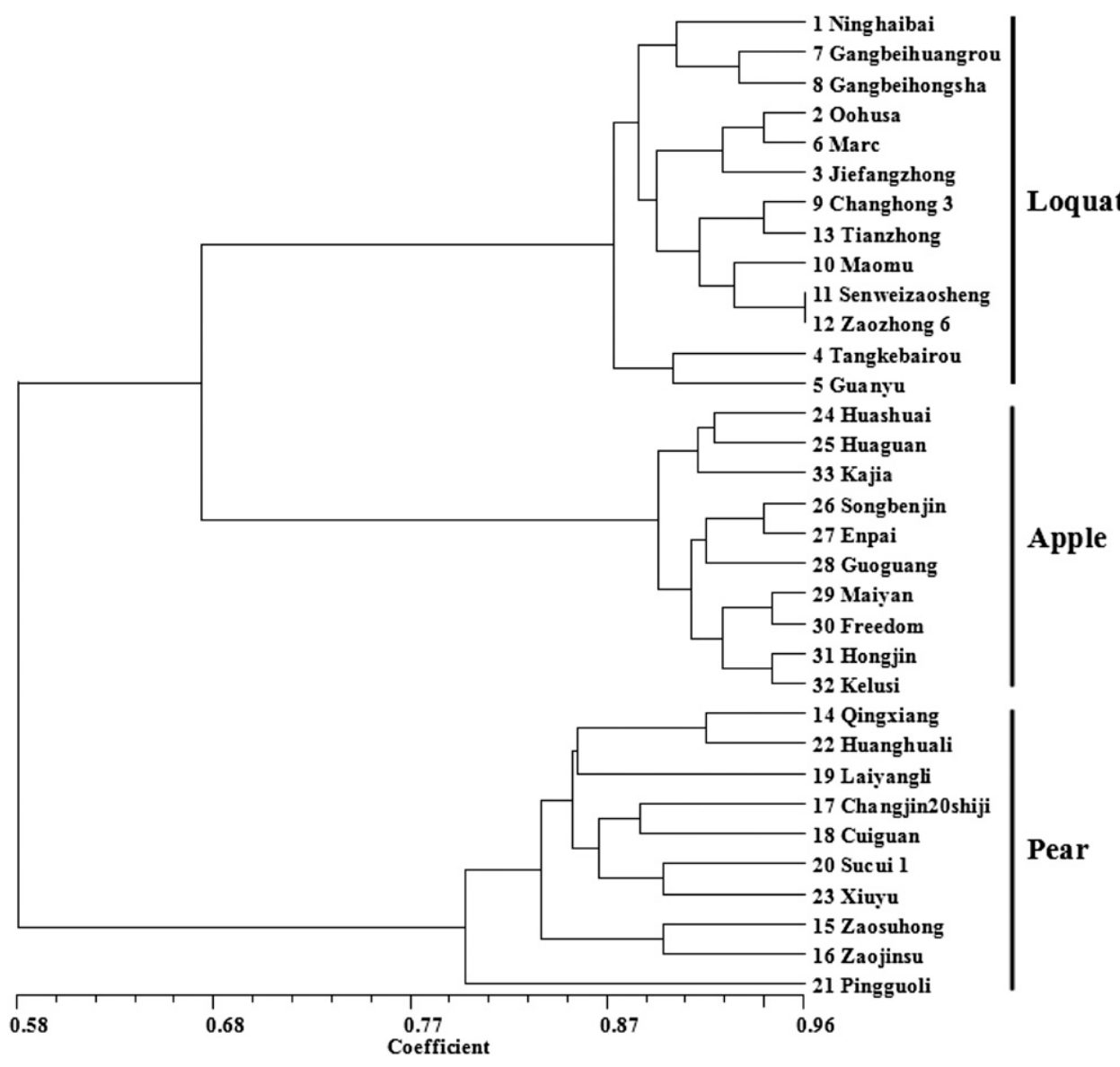

Fig. 3. Dendrogram constructed from 33 cultivars including loquat, apple, pear using polymorphic genic simple sequence repeat (SSR) marker developed in this study. All the cultivars were obviously separated into three groups: cultivars on the top belonged to the loquat group (one to -13), cultivars in the middle belonged to the apple group (24 to 33 ), and cultivars at the bottom belonged to the pear group (14 to 23).

and pentanucleotide repeat units comprised only $4.44 \%$ and $6.07 \%$ of the total, respectively (Table 2). This trend is consistent with the results reported for other plant species (Li et al., 2010b; Liang et al., 2009; Luro et al., 2008; Moccia et al., 2009). In addition, the most abundant dinucleotide and trinucleotide repeat motifs were $\mathrm{AG} / \mathrm{CT}$ and AGG/CCT, respectively. Similar results have been found in other plant species (An et al., 2009; Jiang et al., 2013; Li et al., 2012; Triwitayakorn et al., 2011).

Because genic SSR markers belong to gene-rich genome regions, they can be exploited for use in the marker-assisted breeding of loquat. Therefore, the set of genic SSR markers developed in this study represents a promising genomic resource. In addition, the length variation of the SSR motifs in this study showed deviation from the normal distribution, which may be the result of the strong convergence of selection pressures. This hypothesis is consistent with the conclusions drawn from previous microsatellite studies on tea plant (Li et al., 2010a; Wang, 2011) and seabuckthorn [Hippophae rhamnoides (Jain et al., 2014)]. Furthermore, most dinucleotide and hexanucleotide motifs occurred more frequently in noncoding regions, whereas other

motifs. Approximately $19.52 \%$ of the transcriptomic sequences possess SSR loci, representing a greater proportion than those found in highbush blueberry [18.24\% (Rowland et al., 2012)], field pea [Pisum sativum, 17\% (Kaur et al., 2012)], tea [Camellia sinensis, 16.67\% (Tan et al., 2013)], Citrus [13\% (Luro et al., 2008)], Amorphophallus [11.8\% (Zheng et al., 2013)], and Epimedium [3.67\% (Zeng et al., 2010)]. Our results indicate that the number of SSRs was sufficiently large to identify markers. The distribution density in loquat was one SSR locus per $3.45 \mathrm{~kb}$, which exceeded the distribution densities observed in rubber tree [Hevea brasiliensis, $0.28 \mathrm{~kb}$ (Li et al., 2012)], Cryptomeria [0.92 to $1.72 \mathrm{~kb}$ (Ueno et al., 2012)], and peach [3.2 kb (Wang et al., 2013)] but were lower than those of Epimedium [6.69 kb (Zeng et al., 2010)], sesame [Sesamum indicum, $4.08 \mathrm{~kb}$ (Zhang et al., 2012a)], and peanut [Arachis hypogaea, $6.22 \mathrm{~kb}$ (Zhang et al., $2012 b)]$. These differences of SSR density may depend on the minimum length of the SSR repeat motif.

According to a previous report by Zeng et al. (2010), the frequency of nucleotide repeats theoretically decreases with increasing length, and dinucleotide repeat motifs are the most frequent repeat type followed by trinucleotides, tetranucleotides, pentanucleotides, and hexanucleotides. However, in this study, trinucleotide repeats [5896 (42.1\%)] were the most dominant SSRs in loquat followed by dinucleotides [2887 $(20.62 \%)]$ and tetranucleotides [2161 (15.43\%)]. Hexanucleotide motifs were found in the coding regions. This phenomenon was also observed in Cymbidium ensifolium (Li et al., 2013c).

POLYMORPHISM AND TRANSFERABILITY OF SSR MARKERS. As a result of the sequence conservation of transcribed regions of the genome, a significant portion of the genic SSR primer pairs designed from the transcriptome of a given species is expected to show a high degree of transferability in distantly related species (Moccia et al., 2009). The transferability of genic SSR markers over different taxonomic levels has been demonstrated previously (Bory et al., 2008; Zheng et al., 2013). In our study, SSR primer amplification of the target loquat genomic DNA was comparable to the $60 \%$ to $92.2 \%$ success rate found in previous studies (Cloutier et al., 2009; Dutta et al., 2011; Rowland et al., 2012; Wei et al., 2011; Zhang et al., 2011). A total of 33 polymorphic SSR markers were obtained, which was higher than the total in mei [Prunus mume (Li et al., 2010b) and significantly lower than that in Amorphophallus (Zheng et al., 2013). The transferability of EST-SSRs among different related genera has been reported in many crop plants (Luro et al., 2008; Wang et al., 2011). The high transferability rate of loquat markers to apple and pear indicate that species in the Maloideae may be more closely related than was previously believed. In this study, the markers from loquat had high transferability rates, $90.9 \%$ and $87.9 \%$ in apple and pear, respectively, which indicated that the different species in the Maloideae may be 
evolutionarily closely related; the homologous comparison results also confirmed this point.

Characteristics of Recovered PCR products. In this study, 37 polyacrylamide fragments representing seven primer pairs were successfully recovered, and $89.2 \%$ (Table 5) of them contained real SSR loci, which was higher than the results obtained in mei $(73.08 \%)$ and loquat $(80 \%)$ by $\mathrm{Li}$ et al. (2013b) and Shangguan et al. (2010, 2011). It is worthwhile to note that the size ranges of most of sequenced PCR products in apple and pear were different from those observed in loquat, and showing lower identity between this three species, these observed differences may reflect genetic divergence among the genera, which will provide representative information for understanding the characteristics of genetic evolution of the three comparative genomics and phylogenetic relationship among these three species.

CHARACTERISTIC OF ASSESSMENT OF GENETIC RELATIONSHIPS AMONG DIFFERENT SPECIES. Until now, little research has considered the genetic evolution of and diversity among the three Rosaceae species of loquat, apple, and pear using SSR markers. In the loquat group, the similarity values ranged between 0.87 and 0.96 , implying that loquat has a rich genetic diversity. It should be noted that 'Zaozhong 6', 'Sengweizaosheng', and 'Jiefangzhong' were clustered in the same subgroup as was consistent with a previous report by He et al. (2011). 'Sengweizaosheng' and 'Zaozhong 6' were grouped together with a high similarity coefficient of 0.96 , which was consistent with the origin of 'Zaozhong 6' as a hybrid between 'Jiefangzhong' and 'Senweizaosheng'. The similarity coefficients of apple ranged from 0.89 to 0.94 , whereas those of pear ranged from 0.80 to 0.90 . The cultivar classifications between apple and pear groups were generally consistent with the genetic background and original geographic distribution data. It is noteworthy that the cultivar Pingguoli in the pear group, which was introduced from Jilin province of China, had a lower coefficient than the others. These results also indicated that the loquat SSR markers were useful in the evaluation of genetic relationships between and within the Malus or Pyrus species.

\section{Conclusion}

To the best of our knowledge, this study represents the first development of SSR markers from RNA-seq in loquat. A total of 14,004 SSR loci was obtained and 70 primer pairs were successfully designed based on the transcriptome sequences, 33 of which were validated and showed high polymorphism in loquat. As a result of the conservation of genic sequences, these markers had a higher transferability across species, indicating higher than expected genetic similarities among apple, pear, and loquat. The developed SSR markers represent a valuable resource for conducting marker-assisted selection, linkage mapping, and germplasm characterization analysis in loquat.

\section{Literature Cited}

An, Z., Y. Zhao, H. Cheng, W. Li, and H. Huang. 2009. Development and application of EST-SSR markers in Hevea brasiliensis Muell. Arg. Hereditas 31:311-319 [in Chinese].

Bassam, B., G. Caetano-A, and P.M. Gresshoff. 1991. Fast and sensitive silver staining of DNA in polyacrylamide gels. Anal. Biochem. 196:80-83.

Bory, S., D. Da Silva, A.M. Risterucci, M. Grisoni, P. Besse, and M.F. Duval. 2008. Development of microsatellite markers in cultivated vanilla: Polymorphism and transferability to other vanilla species. Sci. Hort. 115:420-425.
Bouck, A., and T. Vision. 2006. The molecular ecologist's guide to expressed sequence tags. Mol. Ecol. 16:907-924.

Chen, C., K.D. Bowman, Y.A. Choi, P.M. Dang, M.N. Rao, S. Huang, J.R. Soneji, T.G. McCollum, and F.G. Gmitter, Jr. 2006. EST-SSR genetic maps for Citrus sinensis and Poncirus trifoliate. Tree Genet. Genomes 112:1248-1257.

Cloutier, S., Z. Niu, R. Datla, and S. Duguid. 2009. Development and analysis of EST-SSRs for flax (Linum usitatissimum L.). Theor. Appl. Genet. 119:53-63.

da Maia, L.C., D.A. Palmieri, V.Q. de Souza, M.M. Kopp, F.I. de Carvalho, and A. Costa de Oliveira. 2008. SSR Locator: Tool for simple sequence repeat discovery integrated with primer design and PCR simulation. Intl. J. Plant Genomics 412696:1-9.

Dutta, S., G. Kumawat, B.P. Singh, D.K. Gupta, S. Singh, V. Dogra, K. Gaikwad, T.R. Sharma, R.S. Raje, T.K. Bandhopadhya, S. Datta, M.N. Singh, F. Bashasab, P. Kulwal, K.B. Wanjari, R. Varshney, D.R. Cook, and N.K. Singh. 2011. Development of genic-SSR markers by deep transcriptome sequencing in pigeonpea [Cajanus cajan (L.) Millspaugh]. BMC Plant Biol. 11:17.

Fu, X., W. Kong, G. Peng, J. Zhou, M. Azam, C. Xu, D. Grierson, and K. Chen. 2012. Plastid structure and carotenogenic gene expression in red- and white-fleshed loquat (Eriobotrya japonica) fruits. J. Expt. Bot. 63:341-354.

Gao, Z., J. Wu, Z. Liu, L. Wang, H. Ren, and Q. Shu. 2013. Rapid microsatellite development for tree peony and its implications. BMC Genomics 14:886.

Gisbert, A.D., I. Lo'pez-Capuz, J.M. Soriano, G. Lla'cer, C. Romero, and M.L. Badenes. 2009a. Development of microsatellite markers from loquat [Eriobotrya japonica (Thunb.) Lindl.]. Mol. Ecol. Res. 9:803-805. Gisbert, A.D., J. Martínez-Calvo, G. Lla'cer, M.L. Badenes, and C. Romero. 2009b. Development of two loquat [Eriobotrya japonica (Thunb.) Lindl.] linkage maps based on AFLPs and SSR markers from different Rosaceae species. Mol. Breed. 23:523-538.

Gutiérrez, O.A., A.F. Robinson, J.N. Jenkins, J.C. McCarty, M.J. Wubben, F.E. Callahan, and R.L. Nichols. 2011. Identification of QTL regions and SSR markers associated with resistance to reniform nematode in Gossypium barbadense L. accession GB713. Theor. Appl. Genet. 122:271-280.

He, Q., X. Li, G. Liang, K. Ji, Q. Guo, W. Yuan, G. Zhou, K. Chen, W. van de Weg, and Z. Gao. 2011. Genetic diversity and identity of Chinese loquat cultivars/accessions (Eriobotrya japonica) using apple SSR markers. Plant Mol. Biol. Rpt. 29:197-208.

Jain, A., S. Chaudhary, and C.S. Prakash. 2014. Mining of microsatellites using next generation sequencing of seabuckthorn (Hippophae rhamnoides L.) transcriptome. Physiol. Mol. Biol. Plants 20:115-123.

Jiang, B., D.S. Xie, W.R. Liu, Q.W. Peng, and X.M. He. 2013. De novo assembly and characterization of the transcriptome, and development of SSR markers in wax gourd (Benicasa hispida). PLoS One 8:e71054.

Jung, S., T.A. Abbot, R.C. Jesudu, J. Tomkins, and D. Main. 2005. Frequency, type, distribution and annotation of simple sequence repeats in Rosaceae ESTs. Funct. Integr. Genomics 5:136-143.

Kaur, S., L.W. Pembleton, N.O. Cogan, K.W. Savin, T. Leonforte, J. Paull, M. Materne, and J.W. Forster. 2012. Transcriptome sequencing of field pea and faba bean for discovery and validation of SSR genetic markers. BMC Genomics 13:104.

Li, C.Q., Y. Wang, X.M. Huang, J. Li, H.C. Wang, and J.G. Li. 2013 a. De novo assembly and characterization of fruit transcriptome in Litchi chinensis Sonn and analysis of differentially regulated genes in fruit in response to shading. BMC Genomics 14:552.

Li, X., H. Xu, and J. Chen. 2013b. Genetic diversity and relationships among 47 loquat cultivars revealed by EST-SSR markers. Sci. Hort. 160:375-382.

Li, X., J. Luo, T. Yan, L. Xiang, F. Jin, C. Sun, and M. Xie. 2013c. Deep sequencing-based analysis of the Cymbidium ensifolium floral transcriptome. PLoS One 8:e85480.

Li, X., L. Xiang, J. Luo, B. Hu, S. Tian, M. Xie, and C. Sunday. 2013d. The strategy of RNA-seq, application and development of molecular marker derived from RNA-seq. Chinese J. Cell Biol. 35:1-8 [in Chinese]. 
Li, D., Z. Deng, B. Qin, X. Liu, and Z. Men. 2012. De novo assembly and characterization of bark transcriptome using illumina sequencing and development of EST-SSR markers in rubber tree (Hevea brasiliensis Muell. Arg.). BMC Genomics 13:192.

Li, S.X., X.Y. Zhan, Y.Y. Wang, and T.M. Yin. 2010a. Content and characteristics of microsatellites detected in expressed sequence tag sequences in Eucalyptus. Chinese Bul. Bot. 45:363-371 [in Chinese].

Li, X., L. Shangguan, C. Song, C. Wang, Z. Gao, and J. Fang. 2010b. Analysis of expressed sequence tags from Prunus mume flower and fruit and development of simple sequence repeat markers. BMC Genet. 11:66.

Liang, X., X. Chen, Y. Hong, H. Liu, G. Zhou, S. Li, and B. Guo. 2009. Utility of EST derived SSR in cultivated peanut (Arachis hypogaea L.) and Arachis wild species. BMC Plant Biol. 9:35.

Lin, S.Q., X.H. Yang, C.M. Liu, Y.L. Hu, Y.H. He, G.B. Hu, H.L. Zhang, X. He, and Y.X. Liu. 2004. Natural geographical distribution of genus Eriobotrya plants in China. Acta Hort. Sinica 31:569-573 [in Chinese].

Luro, F.L., G. Costantino, J. Terol, X. Argout, T. Allario, P. Wincker, M. Talon, P. Ollitrault, and R. Morillon. 2008. Transferability of the EST-SSRs developed on Nules clementine (Citrus clementina Hort ex Tan) to other Citrus species and their effectiveness for genetic mapping. BMC Genomics 9:287.

Mardis, E.R. 2008. The impact of next-generation sequencing technology on genetics. Trends Genet. 24:133-141.

Moccia, M., C. Oger-Desfeux, G. Marais, and A. Widmer. 2009. A white campion (Silene latifolia) floral expressed sequence tag (EST) library: Annotation, EST-SSR characterization, transferability, and utility for comparative mapping. BMC Genomics 10:243.

Nei, M. 1973. Analysis of gene diversity in subdivided populations. Proc. Natl. Acad. Sci. USA 70:3321-3323.

Ono, N.N., M.T. Britton, J.N. Fass, C.M. Nicolet, D.W. Lin, and L. Tian. 2011. Exploring the transcriptome landscape of pomegranate fruit peel for natural product biosynthetic gene and SSR marker discovery. J. Integr. Plant Biol. 53:800-813.

Pazos-Navarro, M., M. Dabauza, E. Correal, K. Hanson, N. Teakle, N. Real, D. Real, and M.N. Nelson. 2011. Next generation DNA sequencing technology delivers valuable genetic markers for the genomic orphan legume species, Bituminaria bituminosa. BMC Genet. 12:104.

Poncet, V., M. Rondeau, C. Tranchant, A. Cayrel, S. Hamon, A. Dekochko, and P. Hamon. 2006. SSR mining in coffee tree EST databases: Potential use of EST-SSRs as markers for the Coffea genus. Mol. Genet. Genomics 276:436-449.

Qiu, W.L. and H.Z. Zhang. 1996. China fruit records-Longan and loquat. China Forestry Press, Beijing, China. p. 91-239 [in Chinese]. Ramchiary, N., V.D. Nguyen, X. Li, C.P. Hong, V. Dhandapani, S.R. Choi, G. Yu, Z. Piao, and Y. Lim. 2011. Genic microsatellite markers in Brassica rapa: Development, characterization, mapping, and their utility in other cultivated and wild Brassica relatives. DNA Res. 18:305-320.

Rohlf, F. 2005. NTSYS-pc, Numerical taxonomy and multivariate analysis system. Version 2.2. Exeter Publ., Setauket, NY.

Rowland, L.J., N. Alkharouf, O. Darwish, E.L. Ogden, J.J. Polashock, N.V. Bassil, and D. Main. 2012. Generation and analysis of blueberry transcriptome sequences from leaves, developing fruit, and flower buds from cold acclimation through deacclimation. BMC Plant Biol. 12:46. Rozen, S. and H.J. Skaletsky. 2000. Primer3 on the www for general users and for biologist programmers, p. 365-386. In: Krawetz, S. and S. Misener (eds.). Bioinformatics methods and protocols: Methods in molecular biology. Humana Press, Totowa, NJ.

Shangguan, L., X. Li, N. Ning, Y. Wang, Z. Zhang, and J. Fang. 2011. Development of EST-SSR markers in apricot. Acta Hort. Sinica 38:43-54 [in Chinese].
Shangguan, L., X. Li, C. Song, X. Wang, Y. Wang, Z. Zhang, and J. Fang. 2010. Development of EST-SSR markers in Prunus mune and its application. Acta Bot. Boreali-Occidentalia Sinica 30:1766-1772 [in Chinese].

Tan, L.Q., L.Y. Wang, K. Wei, C.C. Zhang, L.Y. Wu, G.N. Qi, H. Cheng, Q. Zhang, Q.M. Cui, and J.B. Liang. 2013. Floral transcriptome sequencing for SSR marker development and linkage map construction in the tea plant (Camellia sinensis). PLoS One 8:e81611.

Temnykh, S., G. DeClerk, A. Lukashova, L. Lipovich, S. Cartinhour, and S. McCouch. 2001. Computational and experimental analysis of microsatellites in rice (Oryza sativa L.): Frequency, length variation, transposon associations, and genetic marker potential. Genome Res. 11:1441-1452.

Thiel, T., W. Michalek, R.K. Varshney, and A. Graner. 2003. Exploiting EST databases for the development and characterization of gene-derived SSR-markers in barley (Hordeum vulgare L.). Theor. Appl. Genet. 106:411-422.

Triwitayakorn, K., P. Chatkulkawin, S. Kanjanawattanawong, S. Sraphet, T. Yoocha, D. Sangsrakru, J. Chanprasert, C. Ngamphiw, N. Jomchai, K. Therawattanasuk, and S. Tangphatsornruang. 2011. Transcriptome sequencing of Hevea brasiliensis for development of microsatellite markers and construction of a genetic linkage map. DNA Res. 18:471-482.

Ueno, S., Y. Moriguchi, K. Uchiyama, T. Ujino-Ihara, N. Futamura, T. Sakurai, K. Shinohara, and Y. Tsumura. 2012. A second generation framework for the analysis of microsatellites in expressed sequence tags and the development of EST-SSR markers for a conifer, Cryptomeria japonica. BMC Genomics 13:136.

Wang, L., S. Zhao, C. Gu, Y. Zhou, H. Zhou, J. Ma, J. Cheng, and Y. Han. 2013. Deep RNA-Seq uncovers the peach transcriptome landscape. Plant Mol. Biol. 83:365-377.

Wang, L.Y. 2011. Mining and application of molecular markers from EST database and transcriptome sequencing in tea plant. Chinese Acad. Agr. Sci. Press, Beijing, China.

Wang, Y.J., X.Y. Li, J. Han, W.M. Fang, X.D. Li, S.S. Wang, and J.G. Fang. 2011. Analysis of genetic relationships and identification of flowering-mei cultivars using EST-SSR markers developed from apricot and fruiting-mei. Sci. Hort. 132:12-17.

Wei, W., X. Qi, L. Wang, Y. Zhang, W. Hua, D. Li, H. Lv, and X. Zhang. 2011. Characterization of the sesame (Sesamum indicum L.) global transcriptome using Illumina paired-end sequencing and development of EST-SSR markers. BMC Genomics 12:451.

Yue, X.Y., G.Q. Liu, Y. Zong, Y.W. Teng, and D.Y. Cai. 2014. Development of genic SSR markers from transcriptome sequencing of pear buds. J. Zhejiang Univ. Sci. 15:303-312.

Zeng, S., G. Xiao, J. Guo, Z. Fei, Y. Xu, B.A. Roe, and Y. Wang. 2010. Development of a EST dataset and characterization of EST-SSRs in a traditional Chinese medicinal plant, Epimedium sagittatum (Sieb. Et Zucc.) Maxim. BMC Genomics 11:94.

Zhang, H., L. Wei, H. Miao, T. Zhang, and C. Wang. 2012a. Development and validation of genic-SSR markers in sesame by RNA-seq. BMC Genomics 13:316.

Zhang, J., S. Liang, J. Duan, J. Wang, S. Chen, Z. Cheng, Q. Zhang, X. Liang, and Y. Li. 2012b. De novo assembly and characterisation of the transcriptome during seed development, and generation of genic-SSR markers in peanut (Arachis hypogaea L.). BMC Genomics 13:90.

Zhang, Y., L. Jun, X. Zhong, F. Li, P. Bo, J. Yu, Y.C. Jing, and J.Z. Xiong. 2011. Characterization and development of EST-derived SSR markers in cultivated sweetpotato (Ipomoea batatas). BMC Plant Biol. 11:139.

Zheng, X., C. Pan, Y. Diao, Y. You, C. Yang, and Z. Hu. 2013. Development of microsatellite markers by transcriptome sequencing in two species of Amorphophallus (Araceae). BMC Genomics $14: 490$ 


\section{Supplemental file}

Seventy unigenes derived from loquat transcriptome used for genic SSR primer design in this study. The details in bold were listed in sequence of unigene code, motif type, repeat motif, repeat size, SSR start position, SSR end position; p2: dinueleotide repeats; p3: trinucleotide repeats; $\mathrm{p} 4$ : tetranucleotide repeats; p5: pentanucleotide repeats; p6: hexanucleotide repeats. The nucleotides of the unigene sequences in pink were repeat motifs, and the ones in yellow were the position of the primers. The left and right primers and the product size designed by Primer 3 were listed below the unigene sequences.

\section{G1_Unigene_BMK.62 p6 (AGCTGG)3 189961013}

GAAATATATTCCATATATGCCTAAGTTTACAG ATATGTTACTGAAGTAGTACGTTCCTCGTCATG A C A G C A C A T A T A T G T A C G T A T A T GC T T G CTTTTATCTTGATTATATCTTTGGATTTTGAAAGAAG AAGACGCTCGGCCGTAGATATTTGCAGCTGTATGAG TTACAAAATTACAATATACAGAATGACATGAG CTACTGTAATGAATGAGTATTACAGCTATGGTG ACTGTAACCCTCCAAAAATGATCTTTCAGACGG TAAGT T TATAATGATGACCCCAGTCTTGATG CTTAAAGCGAACTTTCATGTGATCTCTCTAATATG AAGATGTTGCCTTTATCATCCCTCCCTAATCTCAGGG TATCATCAACATATCTGAAGAAATTGAATTAAAG AAAAAATATAATCTCTACCATTCGAAAATCAG AAAATCTCAAGCAGGATTACTACTACGTCAAAGTGG TAACT T T A GGA T ACGT GATC TCAAGCCATC CTTCCGGGTTGAAGATCCCAAGCAGAATATCA TAATTTTTCCGGAACACGTTCATTAACTGACTCGG A GTGATTGTGGAGTT GTCATAGTTGATGTCA ACTCTTGAATTGGATGCTT TCTTGAAGGAGG CCTCAATTGTCAGCCGTCCATTAAACAGATT CAATCCTCTCACGTCGAACTTGATCACATTAACTG CTTTACCCTTAGCAATGTTAATATTCTGGAAGAAG TCACCCAAGGAGATGAAGTCCCTCAATCCCAG CTTTGTTCGTTTTGAACCTAAAATAGTAATTGTACTG TACACAAGTTTCCAGCATCCACCCACCCTTTGTAG ATTAAGAAGAGGATCTGGAGTAGGATTCCGAG ACTCGAGCTGGTTGACGAGAGCTTCAATCTGAG A T T T C T T C GC A G A C G G G A C T C C GA A A A T G CCTCTATTGATTCCTTTGATTGCTTCCAAAAGCTGTG TCTTAATAACCTCATCATCACCAACGTCCAAGCTGG A GCT GGA GCT GGA A C T T T C T T C T GCA A C T G ACCTCACTGTGCAAGTGTGTCGCCATAACCCAA CAAATCTCGTGCTTTGATGTGCGGTCGAGACCAAA CAGCGAGCAGGTGCCGCCATGTTTCTGGGTCTCAG TCTAGGCACTGGCGGAAATGGAATTGCATGG ACAAGCACGCTGGCCATCAATCTTCCAATGACTCG A C G GCA C C A GA G A T G T T C A C T GC T T GC T A G TAATCCCCAAGCTCATGGTCCATGCCAGTGAGAGGG ATGGGGGAAACCTTACAGCTGCTGTGTGACGAGAG CTTTTAGGCTGTCCTTGTTTGTTTGTCTGTTTTTCTCA TAGCAGCATGTGTTTG

LEFT PRIMER GACTCCGAAAATGCCTCTAT RIGHT PRIMER GTCCATGCAATTCCATTTCC PRODUCT SIZE: 252

\section{G1_Unigene_BMK.64 p4 (TTCT)6 24974997}

AAGAAGTTTTAGGATCAAACATTTTCATAAATGCAG TTGAATTTTATCGCACATATTTTACAATAAATG
TTTAGTCGAGTCTCCTGTCACAAATAGATTATAG CACAGGCAGGCACACCTAAGAGTGTTTTTACA CAA A T G G A A GCA G T A G A C A GA C G T T C C A G TATATCTCTGTTGCCACGACGGAATGGATTCATCGG TCCAGCATCTCTTTGTTTCTAAGAGGCGGACAG ATTGGATATGTGTTCCGTAATGCTAGCTTTGTTCG AATTACAGGCATGAAGCGCAGTGCATACGTCCGTTG T T T C G A G G A A T T G C T C T G C A T T G G T A A G AATGGGGGGGCCATACTCGAAAACGTACCTCTTG CACTTTTTGGTATAGTTCTCCACCGAATTGCATG CCTTCAGAAGCAACTCAATAATCTCCAGCTTCGTG TCGGGATCCTTCAACTTAACCAACACCTCTGAAACAG CACGGTGACATAATCCACAGCTATCCTCTTTGAATTG TGAAGAAAACAAAGCTACTTGCTGACAGAGATTG ACCTTCCGACAGAACTCAGAAGGTTGTAGAGAGG TGGCCTCTAAGAAGAAGAGAGGAGCATAATAG TCCACCAAAGTGACACACTGCTGGCTGAATGAGCG CAAATGATGGCAGGCCTGATGTAGGTATTCAATG ATCTCCGTCTGGGTCTTGTTTTCACTAATGTAAT CAAGTGCTTGGTCAGCAAACTCCTCACACAATGTG CACACGTTATCATTTCCGACAACTTCTTCAACTTGG AAAGTTTGAGTTTCCAACTGGTGGTCTGACTTTG AAACTGACAGATCAAGGTTTGTCAGTTGTCTAGCAT CACAAGCCCAACCAGCCGCCAGCGCAAAAAGAAA CAAAACCCCAACTCTCACGTCCATGGTACCTGCG T T T G T GA GGA GA GA GA A A GA A A GA G T G G AAAACTTGATGTCTAGGAATTCCTTCTTTCTTT CTTTCTTTCTTTCTTTGTTTCGAAGGGTTGGGGG TGGGCGTTTGGGGGAGCGTTGAATTGTCG

LEFT PRIMER CGTTTGTGAGGAGAGAGAAA RIGHT PRIMER GACAATTCAACGCTCCCC PRODUCT SIZE: 126

\section{G1_Unigene_BMK.101 p4 (TGAG)6 2418631886}

GCATTTTTATTACATCAGGAACACACCACTCGCAATG CAAGTACTTACATGGTGCCATTTCGAATCATTCA CA T C CGAA T T ACC T GTAAA T C CA GCA T C T T G TTTATCGATAGAAAAGTACAAGACATTCTACTCAG CTTTTTCCAAATTACACAAAAGCATCAAGGACG AAACTACTACAGGATTTAAACTTTTTGGGAGTGACG ATTTTGCGCTTGTATAACTGATAAGCAAAAGCCATAT CACCAGAAGAGTAACCTATCCCTCGGAAGCATA TTAGTTACTGCACCCCACTCCATCTAGCCCAG TCTACGAACTGCATCCCCCCATAAACATTGTTTG AAAATCGTACTCCGACAGTGAAAGCCATTGCAA CAAGCGGGTTCTGTTTCGCCAAAGGCGATGCCTCTAC CAAGCGTTCAAGTCCATTGATGATTTGATAGCGGG TATTGGAAGAGACAGCAAGGAAAACACCCCAAAG AGCTGCACTCTTTATTAGAGGTGGAACAGGTATG TCCTCTTCTGACTTCTTTATGCTCCGCTTGGCAG TCATGATCAAGTTTGCAATTCCTTGGCCTATAATAC CACATCCAAAGCCTACT GCTCCATACAAAATG CCCTTAAAAAAGTAAGTGGCAATTCTCTGCTTTACAG AATATCTACATCCTGGCCTTTCCGCTTCAAATA CACTGCTAGGAAGAGCTCCATAAGAATGTTGCAAG CGTCCAAGCAACCCTTTAGATAATGATGGTTTCCCAA TACGAGCATAGGGGGCCAACATCCCAACTAAAGCAA CATTAACCACCACCCCAACCAAAAGATCTGCAACA T A C A GC T C A A A T T C T G C C C A GAAA T C T T T G CCCCTCTTTTGAACTTCCGCAAATGTAGCACAACAAG AATCAATGACTATCTCTGTTCCAATTTTGAAGAG 
AAATGATGGATCAGCAAGCATTCGATCGCGAAG CATAGAGCATGACCTCATTAAGAAACCTAGAGG CCAGGCTGACCCCTGCAAATCCAGATATCTAAGGAG AAGCACT T T GCGAAT T CCCACGCTC T T GGCAG CCTCAATCATATCAGAGGGAAGCCTAGCCCCTCGAG CCT TAGTCTCT T TCATAATCTCT TCAAAT T TCA TAATTGGCCCAAACTCTTCTTCTTCTTTATCACCCC CACCACCGTCACCACCACCACCACCTCCTCCTC CACCTCTACTGCTCGTATATTTACCATTACCACCAC T A C C A T C A A T C A T A T C C T T A T C A C T A C C A C CACTCTCGGTTTTTGATCCACACTCATTCTCCG AAATCCTCATTTCCTTTTCTACAATAACATCCCG TCCCTCATTCGAAACAGCTATTGTGCCTAACTCGG ATTGTGATTCAGCCCGAGCCTCGGACATAGACATG ACCGTAAATTTCCGGTCTTTCTTCACCGAAAACG AATTCAGCCTATCTCTAAAGCTACTATTCCTATAGAG CACTGGAAAAGCAA C C TCC T T T GCA T CA T TCC TAAACCTTATTCCATAAGAAATCAAATTCTGACTCCA CATCTTCCTCCGAACGACCTCGTTCCGCAAAGTTG CAACATTCGATATCCCAAAGCTCGAAGAACAAGCCG CCATATATGCCTTGGAGATCTCAAAAATGTCTAAG TCGATAATGCACGCCCAGAGAAGTCGACGAATTAT TACGCAAGTGCCACAAACACAATCTTTAAAGTCAGG CAAGAGGTGATTGCGCGGAGATAACGGAGGAAAGTG AGAGGGAAAGTGGGTGGAAGGTAAAATTGGTG TTTGGCTGCCGAGAAAGTTGAGGGTTTGAGGATTCG ATTCCGAAAGCGATAGCTGGGATTTGGTAGGTGG TTGAGTGAGTGAGTGAGTGAGTGAGGGAAGCGG AAACTCGAAAGAGAGACGACGTGGTTAATGGG CTCCTTATATCCTTT

LEFT PRIMER AGTTGAGGGTTTGAGGATTC RIGHT PRIMER ATAAGGAGCCCATTAACCAC PRODUCT SIZE: 128

\section{G1_Unigene_BMK.137 p3 (AGA)6 188198}

A A G G A A C C C A C C G T C G C T T T T T A T C C C T C CTTCСTTTTCCCTTTAATCCCCAGGAATTCGACTCTG TTCCCTCAGAAATCAGAAGAAGAAGAAGAAGAAAA T A A A A A C C A A A C A G A A G A G A A T G G G G C T G CTCTCCAACAGGGTGGATAAGGGGAGCTTGAAG CCAGGGGATCACATCTACTCTTGGAGGACTGCTTATA TATATGCCCATCACGGCATCTATGTTGGGAATGACA CAGTCATTCATTTTACCAGACATGGACAAGAAG TAGGAACGGGGACAATGCTGGATCTTCTCTTGGTTAG C T C A G G G C C A G C C C A A C C T C G A C A A C C C T G CCAAATTTGCACTCCACGGGAAGAGGGCAGTGGTG TTCTCTCCTCATGCCTGAACTGCTTTCTTGCAGGTGG CATCTTGTATCGTTTTGAATATGCTGTTAACCCTG CTCTTTTTATTGCAAAAGCACGCGGCGGAACCTG TACCCTTGCAGTCTCAGACCCAGACGATGTTGTGG TCCATCGAGCAAAATACCTGTTTGAGAATGGCTTTGG T TGCTACAACGTGTTCAAGAACAACTGTGAAG ACTTTGCTATCTACTGCAAAACGGGACTGCTTG TTCTGGATCAAGGAACAATCGGGCAAAGTGGCCAAG CAGTATCCATCATAGGGGGACCTCTTGCAGCTG TTTTGTCCACGCCATTGCGTCTTGTCACAACCAATG TTTATGGGATGGCGGCAACTGCCATTGGAGTCTACTG TGCTAGTAGGTATGCTGCTGACATTGGCATGAGG AGGGATGTAGTGAAGGTGTCGGTCGAGGATATG ACAAGGAGAGTGGCAGCTGGCCTGCTCCAGGTGG CCGAGGCCCAAATCACAGCGGCCCCAATCACGG
CCCTTGCAGGAGGTTCTTCGAACCTTGTCTCTTG ATAGTATGATTGTTTTTATCACATTAATAAGTACTTG TGCAATGACATCTGCTGTTTAAGCTGGCTTGTATG ATGAGATGTTTGTGTTTTCTTCATGTTGTCCACCGG CATTGTGGAGTTTATAAGGCCAAGATTGGTGG CATCTGCCTTATTCAAAAACCTTCTTTGTAAAATG ATCGGTGTGGGATATTTTTTTTTACTTTATTCATG ATCATACTTACTAAGGTACCGAAGTATAACACCTC TAGATTTGTACCACACATTTCTTTTTGTTGGATA CAAATGATGTTGGGGGAGGGGGAAATCGAACTCGTG TTGGATACAAATG

LEFT PRIMER TTTTCCCTTTAATCCCCAGG RIGHT PRIMER TAAGCAGTCCTCCAAGAGTA PRODUCT SIZE: 164

\section{G1_Unigene_BMK.160 p3 (TCA)7 21242262}

GCACGCATTTTTTTTTTTTTTTTTTGTAATGTATAAA TACTCAAATATCATATAATCTTGAACTTATGTACCAT CATCATCACCACCATCATCATCAAGTCACATATCG AACCAAATAAGAAAAGAACGAAAAATAAAATA AAAACAGAAAGATT TGTAAAAACTCTCTCTC TTTTTTCCCTAGCGATGAATTAATCCATGGCCAAATG AAAACCAATAATAACAATCATCTCT TCTCAT T TCATCATCATCATCATCATCATCTTCTTCTTCT T TATCTCTCATCCTTCATTATAATCTAAGCCTGAAGCG AGTTAAACGCGTCGCACCTGCGCCTCACCTCTCCCTG A T G C C C GG T C T T A A T C T C A T GA T G T C C A A G C T T C T C C A T T G C A T G A G A A A A G C C T T G A AAAACTCCGCCTGGTCAGTAAAGTACAACTCCA CAAA A GGC T T CG T CC TCGGGTCCT TAGCAAG T G C A T G G T C C G A T G C C A G C A G C C C C A A G CCCCTCTTTAGAATCTGAAAGTACATGT

LEFT PRIMER ATCCATGGCCAAATGAAAAC RIGHT PRIMER TGAGATTAAGACCGGGCATC PRODUCT SIZE: 174

\section{G1_Unigene_BMK.217 p6 (TCCTTC)3 1894111}

TAAAGCCCTCCTTGAAACCTCAGACATTCAGCTTTTG TCTGTCCTTCCTTCACACAATGTCTCTGACACCAA T A T C T T T G C T C A C T C T C T G T A T C C T T C T C C TTCTCCTTCTTCCTCCCACCGCCACCTTAACCCTCCA CAACCTCCTCCAAAGCCAAGGCCTCCCTGCCGGG CTCTTCCCGGCCAATGTGAAGTCCTACAATCTCAAC CAGATGGGCCGCCTGGAGGTGCATCTGGACCG T T C T T G CA T G A C C A A G T T C GA G A C A A G A G TCTACTTTGAGAGCATAGTCAGAGCTAATCTCAG CTATGGCATGCTCAGAGGCTTGGAGGGTTTGACT CAGGAGGAGCTTTTTCTTTGGCTGCCGGTGAAGGG AATCTCAGTGAGCGATCCGTCTTCTGGGTTGATTTTG T T T GACA T T G G T G T T GCCCA T A A GCA GC T T T CTCTCTCTCTATTTGAAGATCCCCTTGTCTGCAAG CCTCAATCTCAAGGCATTTCATTGAAGAGTGAAGG AGAAAGGAAGATTGGATTTCAGGCCCAGAGATGAAG ATTTGATCTTTTGTCACTTGGATAATTTTTCTCATG TTTTGCTTTTTGGGTATTTTTCTCTTTTAATTTTGAG CAAAAAAGATTGAATGTTCATGGAAGCTTGTTAGG ATTGTAAAAACAAAGGAAAGCAAAAGTGTGTGTAAG ATTTTGAATGAAATAGTGCAATTTTGGAAGCTTG CTCAATTGGAAATTTTCGGGTTTTATATATTTTTG TCTTAG 
LEFT PRIMER TTCAGCTTTTGTCTGTCCTT RIGHT PRIMER CTTGTCTCGAACTTGGTCAT PRODUCT SIZE: 242

\section{G1_Unigene_BMK.254 p4 (CATG)5 20352371}

GGCGGAGTACAGGCCGGAGCGGTGGCTGGAGAG AGGAGAGGAGAGAGGGACTCACAAGAAGTGGAAG T T C G T T G G T A G G GA CA CA T A T A GC T A T C C GG тTтTтCAGGCGGGGCCGAGGATCTGTTTGGGGAAGG AGATGGCGTTTTTGCAGATGAAGAGGGTGGTGAG CGGGGTTCTGAAGAGGTTTAAGCTGGTCCCGCGGG AGAGGGAGGGTGGGGATGAGCCGGAGCTGCTGG CCTACCTCACCGGGAAGATGAAAGGCGGCCTTCCGG TCACCGTTTTGGAGAGGGCTTAATTAACTATATATTG TCAATGTGTGTGTATCGTAATGATTACGTAGGTAAT CACATACATGCATGCATGCATGCATGTAGATGCTTAT TA T T T T T GAA T T T A AC T T TAACT T T G AA TATTTGGTCTTATCATGTACGTGTCTCTAAAGAG TTTGTACTCTCCATAGTTATATGGATGGATGTTTTG ATGTTTTTGGGCACTTGGT

LEFT PRIMER CTACCTCACCGGGAAGATGA RIGHT PRIMER GAGACACGTACATGATAAGACCAA PRODUCT SIZE: 204

\section{G1_Unigene_BMK.267 p4 (AACC)5 20205224}

TCGGTCACTTAACGCTGCAATTCACAAGACAAATC CAGATCTGAGACTAGCCATTGTAATTGTGTGATG T T A TCT TCCT T TCCATATATTAGCAAATGTAA AAAAGGCATCAAAAGAAGATTACAGAAGAAAAA TATGGATTAATTACCCAATAAACACGATCAAGGCCG CTACGACACACAATCATATATTGTAAAATTTACAAAC CAACCAACCAACCAACCTGGAGGGTGGATTGTG TTCATTAGGATCAATCATCAATCCCTAATGCCAAG CTCAACCTCGAGTTCATCGTCGTCTTCAAAGAG C T T A A G A A A C G G G C G T G T T G T C T T C TCTCT TCT TCT TCTGCCATA TCT TGAACA T G AAAACGCAGAAGCCGATGACAGCCGCCACCCCCAG C A G C A C A A T C A C T G C C T T G A G C G C C G C C G ACCCACTTGAAGCTTTGTCTCCTTTCTTGGGAG TGGGAGTTGAGGGCTCTTCTGCCGAACCTAAATCG A A G A G G A G GC T A A T GGACA A G A G A G G A ACCCA T T T GCA GCA G T T CA GACA T C T T T GG CAATGGCGGCTCCGAAGTCCATTCATGTCGTCTG TCGTGTTTCTCGCGGAACCCAAATAAATCAACG ACGGTGAAGATGTCGTACAAGTACAAATACAAATA CAACGTTGAATTTTCAACTGCAATTTGGAAGTGG ACGGGGAATGCTCTCCGTTGTTTCCTTCCAACTAC TAAACGTCAAACACTCACTCCAACCTAACGAATG TACGGGTCACCTGCTGTCGTACAAATTTCCTTTTA CAATTCGGAAGTCTA

LEFT PRIMER CAATAAACACGATCAAGGCC RIGHT PRIMER TTTGAAGACGACGATGAACT PRODUCT SIZE: 156

\section{G1_Unigene_BMK.328 p2 (GA)10 2019972016}

AGAAAAGAAGAAGCAAAGGCAAAGAAGATTCTTG ATGTCATTGTTTCTTGTTACAATCACAAATTTCTTTA TACCAAATCCAACGGCTGAGATCTACAACCTTTC C A G T A G A C C C C A C C A C A C A T T A T T A C A A C CCTCCTCCCCCCATGTTTCCAGATCTACACCTTATG T C C C C T T C C C CA T A T T T T T C T A A A CA T T T T
TATCTTCTTTTTCTTCTTCTATATGAAATTATATATG ATACATCAAGAACAGCTGCAACATTTTGGATCCAAT T A C A T C C A G G A C C A C A C A C C C A A A T T A C T TAAAAAAAAAAAAAGTAAAAACGGAATTTAATTCAA CAAAAAATTTAGACAAAAGGCCATGTGATCGGTG ACTTGATGATTTTATCAACCGGACTCGTCGGTATG CTGACCGCCCTTCTTCCCGCTGTCCATTTCCTTTTT TACCCTCGTCTCGATCCCGTAATAACTGACGTAC C A C T T G A C G A A C T T C C T C A G C C C A C T G G CCAAATCCGTGGTGGGTTTGTACCCGAAGTCCTTG TACGCCAGGGTCACATTGGCGTGCGTGTAGGGCACG TCCCCGTTTCTCGGCATCTTGATCACGTGCTTCCTCG CCTTCGTGCTTAGAAGCCCTTCCAGTATCGACAC CAGTTTCCCCACCGGCACCGGCGACGTGTTGCCG A G G T T G T A A A T T C G C A G C T G G G C G G G G CСТTTCTTTTTGCCCCCACTTCCCGTGCTTTTTTCGG CTGTGTCCAACGCTCCCAGGCACCCTTTTACTACG TCGTCAATGTACGTGAAGTCACGTGCCACTTCCT T A T C G T C C A C T G T T T T G T A GA C G T C GA T C G G TTTACCCTGCAGTATGTCCTTGGTGAAAAAGAAA T A C G C C A T G T C G G G T C T C C C C C A A G G T C C G TACACGGTAAAAAATCTTAAGCCGGTGAGGGAAAG TCCGTAAATATGATTGTAAGTGTGGGCGATTTCCT CACCGGCTTTTTTTGTTGCCGCGTAGAGACTGG CCGGTTGGTCAGTCCTGTGCAGCTCGGAAAACGGG TTGTCGGTGTTGAGCCCGTAGACCGAGCTGGACG A T G C C C A G A C A A T G GA G G GC T GA G G A T T T G CTCTTTTTGCCACTTCGAGGAGATTGACGAACCCGG CAATGTTGGAGCTCACGTACGACTGCGGGTTCTG CATAGCGTACCGGACGCCAGCTTGGGCCGCCAGG TGCAGGATGTGCGTGAACGGCACCACATCGAACAG C T T C G T C A GCA GC G GC T C G T C G T T C A A G T C C CCCTCCACGACGAACACCTCATGCTTCTTCAG CAGGGCCTGCCTCGACCGCTTTAGCGAAGGGTCG TAGTAGGAGTTGAAGTTGTCGAGTCCCAGAACTC CATCGCCCCGCTTTTTTAAGGCGAGGGAGCAGTG AGACCCGACAAAGCCGGCCGCGCCGGTGACCAG CACCGACATGCCGTTGGGGCGTCTAGGAGTGGCAG A C T T G C G G A C C T G T T T T T C C C A A G C A G T C C СТСССАСТCCСCCACCGTAGAAGGCGGAGGAGAG A A A G T T G T G A G T G G T G T G G A C G T G G T T G G CCCCACCGCCGACGTGATCGGAGAGCGGAGGG TAGTTGAGGGTGAAGAATAAGATGAGGACAAGGG CGACGAGGAGGGTGGCACGGAAGAGGAGCTTGG AGGAGGCGTTGAGAAGCTTGGTGCTGTTGACCCTG CGGAGGTAGCTGTTGTAGCGCTCCAGCTTTATGG T C T T G C T T G T A T C T G G C G G A G A A G C C A T T G CCAAAAACTTACACCAAAATATACAGAGAACC CAAAATCCAAAGTATATATGAATCAAAATACG AAAATATATGTACCCAAATTGTGTAAAAACAAAAG AAAGGGTTTTGTGCTTCGGGACAGAAGAGACGG A TCTAAAGACAAACGAAGGGACTCAGAGCTG AAATGTGAATGAGCCCTGAATTTGGGGGGAGTG CAGAAAGATGAATATAAAGATGATGAGAGAGAG AGAGAGAGAGATGCAATAACAAATTAATAACAA CAACGA GCT T T GCTGTGTAT T T TCTGT TGT TG CTATTGTGCCCAGTAACCCTTCTCTCTCATCATCAT C A T G G A T C C A T A T C T A T C C A T C C C C T T C T

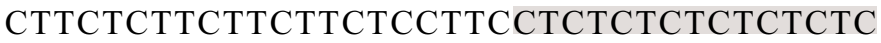
T C T C T T G G T T A A C T C A G A A C A A C T C T G T T T TGGGGGTAGAGCGAGAAAGTTCAAGCCCTAGAGG TAGAGAGAGAGGATATTCAGTTGCCCTAGG 
LEFT PRIMER ATGTGAATGAGCCCTGAATT RIGHT PRIMER GCACAATAGCAACAACAGAA PRODUCT SIZE: 138

\section{G1_Unigene_BMK.328 p2 (CT)10 2021572176}

LEFT PRIMER ACCCTTCTCTCTCATCATCA RIGHT PRIMER CCTAGGGCAACTGAATATCC PRODUCT SIZE: 180

\section{G1_Unigene_BMK.407p6 (CCCATC)4 245679} TCCCGCT T TC T T GGACCTGTAAA GGCATATCC TCTCCCСТCCTTTGTTCCATGCTCCCATCCCCATCCC CATCCCCATCCAAATTAAAATTTTCATTTAAATTTG ATTTATTCATTTTATGATTGTTTTGGTAGTTTATTATG CATCTGGATCAGATGAGATGAGATGAGAGAGGATA TAGTTAAAATTGGATATGTGGTGGTTGTTTTAGTTG AATTTCTTTCAGATATCTCGTGCTACATTTCTTATTG CATGTCACTTTTGAATGAATGATATATTACTTTGG TATCTTGATTGATGTGGACGATTGAGTTGCTGCTTGG TAGTTTAGCTGTTAAAACATGTATGTATATCACCTTG CTTTTTTCGTTTTTTGTTAGTGTACCTTTTGTTA TATGGTATCGTTGTTGGGTTTTGAATGATGTTTAAT CAGCTTCGGATTCAGGCATGACGTACACCAGCCCTG CTGCCAGCGCCAGCACCAGCACCAGCAGGGACAG ATGAAAATCTTTAGGGCTTAGGGTTTAGTTGATTG CATTGTCTTGTGGGCTGTATGCAATTAATGAATT CAAAGTGTAGAAAGATTACAATTTGTAATGAACAAG AATTCTTTTAATGTAAACCAGCTGTAACTTTGATAG TTTGACAAATGGAATGGAATGGAAGGGCGATAG CCAAAACTGATTCATGCTCTATTTTACTAGGTG CAAAATGAATTGGATCTTTG

LEFT PRIMER CTTGGACCTGTAAAGGCATA RIGHT PRIMER CAACTAAAACAACCACCACA PRODUCT SIZE: 205

\section{G1_Unigene_BMK.407 p6 (CCAGCA)3 18476493} LEFT PRIMER CGTTGTTGGGTTTTGAATGA RIGHT PRIMER CCACAAGACAATGCAATCAA PRODUCT SIZE: 146

\section{G1_Unigene_BMK.436 p3 (TTG)6 1810761093}

GGAGAAAAGAAAACAAAAGGGCTCTGGAGCAGTGG CTTTTGGGAAGCAGAAAGAATGGTAGAGGAGAAG CCTGAGGAAGAAACCATGGTAGAGGAGAATATGG AATTATCAGAAGTACCCTTGAAGACTACTCATTTTG TGCTGGTTCATGGCATAAGTCATGGAGCATGGTG CTGGTACAAAATCCGGTGTCTCATGGAGAATTCCGG CTACAAGGTATCGTGTGTCGATCTCAAGAGTGCCGG CA T C GA T C A A T C T GA T G C GA A C T C A G T C C T T TCTTTTGATGATTACAACAAGCCACTCCTGGACTTG C T G T C T A C T C T C C C T G A G A A T G A A C A G G TAATTCTGGTGGGACACAGTGCTGGAGGGCTGAATG T A A C T C A G G C C A C T C T C A A G T T C C C A A A G AAAATTCTCTTAGCAGTATATGTTGCAGCAACAATG CTCAAACATGGGT TCTCCACTGATCAAGATT T CAAAGATGGTGTGCCAGATTTATCAGAGTTTGGTG ATGTATATGAGTTTGGGTTTGGATTGGGAGCTG ATAAACCTCCAACAAGCGCCATTGTCAAGAAAG A A T T C CAA C GCAAA A T C T CA T A C CAA T T GA G TCCTCAAGAGGATTCGACGCTAGCTGCCATGCTTTTG A GGCCA GGGCCGCTCT T GGCA A T CACGTCAG CCCATTTCGAAGGGGACGGCGTCATCGACCAAGTG
CCACGAGTTTACGTTAAAACGCTGCATGACCATG TCGTGAAACCAGAGCAACAAGGTTCGATGGTAAAG AGGTGGCCGCCATCGGAGGTCTATGTTTTGCATAG TGATCACAGCCCTATGTTCTCCACCCCATTTCTG CTA T T T GGCT TCCT TGTAAAAGCA GCGGCT TC TTTTGGTGGACTTAAATAGTAGCTAGGACCAGGAG CCTAGGATTTTTCATTACTTCATTGGAAAAAAATT CAAATAATATTGGGGAATAAGAACATATGGAG CACTTTGAGCTAACGGAAGATTGGGTGTAAAATCG AGCGCATTGGCGTTATAGGATTTATATCGACCACACA TAGTGGTGTGGGATACGGCTTGATTGTTGTTGTTG TTGTTGTAATAGGGGAATAAGAATAATCAAATG TTCATCAATTGGATATGTGAGACGCTAAAACGACCG TAATGGCATTATATGATTTATATAGCTGACCACACT TAATGAGATAAGGTTTGGTGGTTGTTG

LEFT PRIMER ACGGAAGATTGGGTGTAAAA RIGHT PRIMER CTCATTAAGTGTGGTCAGCT PRODUCT SIZE: 207

\section{G1_Unigene_BMK.458 p6 (GAATGG)3 18303320}

CAAGCAATATCCATCACACAATCAGACAAGATG ACTAGGACTGAGCGAAACATCTCCCCTGCGGCTTTG ATCAAGGATCGATCCGTCTCCAAATCCGGTCTTG ACAAGTCCCTCAAGAAACAAGGTGCCGGTCCCCA CAACTGGGGCTCCATCACCCAAGAAACCGCCGGCTA CATCCACAACCCCGACTCGTTGAACCAATTGTCCG CTGACGAGAGGATCGGTGTTGACGATGCTGATGCCG ACGACGCCGATGCCGATGCCGAGGAAGAGCAG AAGGAGGGTGAAGACAAGGTTGAGGAGAATGGG AATGGGAATGGGATCGATATCAAGAGGAATGG ACCGGTTCCGAGGAGGATGAGCTCGATGAGTGAGG AGGAGAAGAAGGAGGCTGCTGCTTGGAGGAAGGG A GCGATGGGGAGGGGTGATGTCGACCTCAAG AACATCGCCCGAACCTCCGGCGCCTTCAGCCAAAG CCCTCCTGAGAACGGTGCCTTGTCCACCTCCCCGG TCAGGAACATGAACACCCTCGCTCAAAAGCTCGGG AAATACTAAACCCCCTCAATCCATGAACCCCACCA C A T C GC GC C A A C A C T T T T G A G C GC C T T T C C G TCCCGGTTACGATCGAAGTCGACCTGGATCGGACA CAATTAACACGTATTCACACATTTGAAAGCATA TTTACATATTCATAGGGTAGATGGGGGTTGAGCAG TTGAGTGAAATGGTTGTTGAACTTTGAGAAGAAAA CAACGTTGTATCACTTAATGAAACAGAAG

LEFT PRIMER GACTCGTTGAACCAATTGTC RIGHT PRIMER TTCTTCTCCTCCTCACTCAT PRODUCT SIZE: 200

13. G1_Unigene_BMK.577 p3 (AGT)7 2119111931 AGTGCTCCCACTCTTTCCACTTCGTTTGCATTGCCAA CAATGTCAAACATGGAAATATTTGCTGCCCCATCTG CCGT TCCAAGTGGAATGATGTACCT T TCCAAG CTCCCACCAACGTCGCAGATGCTCAACAAAAC CACTTGGGTCACATCCATAACAGTTTTAACCATCG T T A CCAGTC TCACC T CCCA T T GCT GCCGCAGG TCCATCCCGAACCACTTAGCTTCTCAGATGATGAG CCTCTCCCGTCCACCTCGCCTAGTCGATTCTCTGG CCCCCAAAATGTTGCAATCAGGACTTATACAGAG TATTCTGCTATCCCTGTTGCAGAATCTCGCCCAA C A T T T C C T G T T C T T G T C A G C G T T C G A G C A C CACCCCTTCAAGGAGGTTCAAACAGCCATGGCCG 
TACCCCCATTGACCTTGTAACAGTTCTGGATGTAAG TGGCAGCATGTTTGGCACAAAACTTGGCCTTCTCAAG CGCGCTGTCAGATTTGTCATACAAAACTTGGGG CCTTATGACCGACTTGCCATAGTTTCATTCTCAAC TACTGCTAAAAGGGTCTTTCCTCTCCGAAGAATG ACTATTGATGGTTGTGAAAGCGCCCTTCTAGCAG TCGAGTCGCTTAGAGCACATGGTGGAACCGACATTG TTGGAGGACTCAAGAAAGGAGTTCAGGTCCTTGAAG AACGCCGGGCAAGGAA TCCA T T TC TAGCAT CATCCTCTTATCTGATGGAAGAGACAATTGCACC TATGACTCACAGAATACTTCAAGTCCAAACCGGG TCTCTGAGTTCTTGAACCAATTGCCAGCTTCGG TACATTGTAGCAATGTACCCGTCCACACATTTGGG TTTGGCACTGACCATGATGCCACTACTATGCATGCTA TATCTGATTCATCCAGTGGCACCTTTTCCTTCATTG AGTCAGTTGCTATGATACAAGATGCCTTTGCTATG TGCATTGGTGGTCTTCTCAGTGTTGTGGCTCAGG A A C T T C G A C T C A C A G T T A G G T C A G C A T C G CACGGGGTGCAGATTGTATCAATACCATCAGGGAG C CA T G T GA G T GA GA T T T C T GA C GA GT G T CAA AAAGGTGCTGTTGATGTTGGAAATATGTACGCAG AGGAGGAGAAACGGTTTCTGGTGTACCTTTTAG TTCCAACATCATCAGCAACTGGAAACAGGGAAGG CGTAAGGAAGACATCACTGTTAGATGTGTCATGCG TGTACAAGGA TCTAGGCTCAAATGAGTCAACC CATGTGCAAGGTGAGAGAGTAGAGATACTAAG ACCTGACGTTTGGTCCCCTGCAGATGAGGCAGTGTG TTTGGAGGTGGACCGTCAGAGGAATAGAATTCTGG TGGCTGAAGGTATTGCAGAGGCACAAGTAATGG C T GA A A T GGGA A A T C T A G A A G G GCA C A GG CTATTTTGGCACAACGAATAGCAACCATCTTAACAT CA C CGGCA GCACA A GC T GGGGA T GG T C T T T G TAATTGGCTGGAAGCTGAGCTTCGAGAAATCAGGG A TAGAATGGCTAGTATGGAATCATACACACAG ACCGGGCGTGCTTATGTTCTTTCAGGGTTGAGCTCA CA T T CA T GGCA GA GA GCCACAAC T A GGGGTG ACATGACAACCCAGATTATGACTATGAATGAAGG TGGAAACTCACGCAGCAGGGCAGTTGGCTATGAAA CACCTACTATGGTTAACATGGTAAGGATGTCGCAG AATCTTGGGGTTAATCGCACGGCTTAACTTCAA C A A G T A A T C G A G C C T C A T A G C C T G A C T T CATTTTTCGGTAAGGTGTGAATACGGATATACAACG TTCATCCTCGGATTAATTCATACTAGGCTGATAG TATTAGTAACTGGTACTTTCAAAAGAAACCTTTTA TAAATTCTGACAGCAGTAGTAGTAGTAGTAGTAG TCGTCGTCTTCATACTTAATCCTGCTGCTTGTTTTG A TGGGTT TCCACATGATTT TCCTTCA TAATTTG ATGGATATTCGAGTAGTTGAGATTCTTATGGGAAG TTAATATTCG

LEFT PRIMER TCAACAAGTAATCGAGCCTC RIGHT PRIMER GAAACCCATCAAAACAAGCA PRODUCT SIZE: 216

\section{G1_Unigene_BMK.673 p6 (GGCACC)3 18342359}

GTTTATTTAAGCGAAGAGAGTTGTGGTCGTCACAG CGTTTACGTAATGTACAGATAATTTGTGGAGACTCG CGCACATCATTCTCCCATCCTCCCAGCTTCACTCAT CAGCAGACACTCACTCCAGTGTCTTCCTTAGTTGG ACACCTCACAGTCCTTGGAGATGTGGCCGGTCTTG CCACAGTTGTAGCAAGCCTTGGCAACCTTGTCGGTGG CCTGGTCACACTCCTTGGAAATGTGGCCGTTCTTG
CCACACGAGTAGCACTTGACGGCGTTGGCCTGGCAG TCCTTGGCAAAGTGGTTAGGGCCACCGCACTTGAAG CACATTGGTCCCTTGGCACTGGCACCGGCACCGG CACCAGTAGCACGTGGCTTTCTGGCGGCTGGAG C T GGGGCG T C C T C GCGGGCGGCG T T C T T A GG ACACTCGCGGGAAATGTGGCCGAACTCGGCACAG TTGTAGCACTTGGCACCCTGGGCGCTGTTTGGGCAG TCACCCTGCACGTGACCAACGTCACCGCAAGAGTAG CACTGCTTGGTGGCGGCAGTCTTTGGCTCTGGGCAGG CGGAAGACTCGTGGCCCAGCTGGCGACAGTTGTAG CACAATCTCTCGGTCTGCTGGCAGTCGTCAGCGATG TGGCCGGCCTCGCCGCACTTGTAACAGGATCTTGGG AACATTTCTATGGTTTTTATGTGTGTG

LEFT PRIMER ACAGTTGTAGCAAGCCTTG RIGHT PRIMER GAGTGTCCTAAGAACGCC PRODUCT SIZE: 246

\section{G1_Unigene_BMK.683 p5 (ATCCA)4 20771790}

GTTGAAATGATTAAGAGACGACGTACTGATCAAA TATTGACTGTTTCATTCAAATACAGACACATCG ATAAAATCCCGAAGAGTGATCATTTATACACGGTG ATTCATAGTCGGGCTATTGTACCTCAGATTTAGG ATTCCCTGACATTTTTATTTTGGTACACAAAAGTTCA CA T CACAACA T T A T CA A ACCA GCCTAACA T T TATTTTGCTAATCCTTCAATCCTACCTTCAGCTCA CAACACACACA TAAACGGAGTGAAGATCCGG AAAATCTTCAAGAGTCGGTGCGGGACTTGACAATG AAATTCTGACGGCTAATGGGATTCATGACCACCGG T G G A C C G G C A G T C C G A G G C C A T G C C T G AAACTTCTTGTCAGTTTCTTCCCACCATTCTTTGTTTG C A A C G G T T A A C G G A G T A C T T C C T C C A A A TATT T TCT TGTCAGCGAAATAATGGTCAGCTG CCCATGCCACTGCAAATGATCCCAAAACAGCAGG A A T GA T G T A T T T T GCA GGCA T C T T C A A A T T T CAAACTGCCAATGACATAACCCAGTTTCGTGAGAG AATATGCAATGACAACAAAATACGAAGGCATT CAAAATATCCCCTAAGGTCACGTTTGGTCCCTTAGG ATTGGTAGATGGGATAACTTACTAGATTCAAGG T A T G C T G A A G T A A G A G A T G A A G A C C G T TCCT TCAACT TGGGCAGAAT T TGGAAGTCAG CCTTCATATCTTACTTCAAGATACTTTGAATCTAGTG A G G T G T C A T A T C C A A T C C A A T C C A A T C CAAACCCGGGCACCAAACATGGCCTAAAACGAAC TAGATAGTTCCCTATCTGTTTGAATTGCCAATTAAG AACGACTAGCCTAGTTCCCTATTTGGTGGCTGGG A C T A C A G A G G G C A G G A A C A A T T A A A A T G G AATAACTGTCGTGGCCTATGCTTGATGAGCTTAACAA T A G C G G G A C T A A G A C T C A G T T A A A T T A G A T A A C T T T C C C A C T T A T A A A G A A A C A T G TCTCCTCCCCAAGGGTCAGGAGAATCTAACATG CAACCAAAGGTATATCCCCCTACTATCAGTATCTA TATCATCGTGTGAGCTATTACTATCTTTCTCATCATG TGGATTTACATGATGAGAGAGA

LEFT PRIMER AACTTGGGCAGAATTTGGAA RIGHT PRIMER GCTAGTCGTTCTTAATTGGC PRODUCT SIZE: 169

16. G1_Unigene_BMK.704 p6 (TTTTGT)3 18702719

TCCСТCCAATCTCCCCAATTTCCACCGTCTCTTCG CCTTCCTCTTCTTCTGCACTCTTCTGTCGTCAACCTT 
CAACTCCCTCCCAACAGTCAAAAAGTCAAAG CCCTAACCACCGTCCGATGACCACCTACGGCACG A T C C C G A C C T C C T C C T C G T C C T C C C A A G G CCCCTCCGGCGAGTACATCTCACGCGCCAAGGACCG CATCAGGGCCGGCCTCGGAGCCCGCCGGCCATGG AAGCTGATGTTCAACTTCCGGTCCTTCAACTT CCССТCCTCCTTCTCCGACGCCTTCGGGCGGGTCCG ATCGAACTATGCCTACTTCCGGATGAACTATG CCATCGTGGTGCTCCTGATCCTGTTCCTCAGCCTG CTCTGGCACCCGATCTCGCTCATCGTCTTCGTCAT CATGATGGCCGCCTGGCTCTTCCTCTACTTCCTCCG CGACGAGCCGCTGGTGCTGTTCGGAAAAACGGTAG ACGACCGCGTCGTTTTGATCGTGCTGGCGGTGGTG ACGATCGTGCTGCTGTTCTTGACCCACGCGACGGTG AATATTCTGGTGGCGCGGTGATTGGGGTTGTGTTGG TGATTGCGCACGCGGCGTTGAGGAAGACGGATG ACTTGCCGGTGACGAAGAGAACTCTGCGTTGTTG ACTACGCCTGCATCTTCCTCTGCTTAAATACTCCG ATTCGATTCTCTGTTTTGTTTTTGTTTTTGTTTTTTTTG TAATTTATGGGTTTTGGTGATCGAAGTTGTTGATTG TATCTGCCAGTCTGCTGCTGCGCCCAATTGTTGG TTTGCGATGAATCTAGTGTTTTTCTTATGGTGG AAAAGTGTTGTGCATATTTGTTCCCCATTTTG TTTCCTCCGTCCTGGTGTGTCTGTGTAATTTGGTTCTG CCTAGTCAAATGGTATTTTTACTTGTCCCGTCCTCG CTCAGGCTGATGAACTATCCGATGAGCCGACCCTCG AGGAAGGATACTTGCAATACTTATATTGACTCG T T T CA T T T GA T A A T T T T T GCT T CA GGAAAG T T C T C C GGTGGGGAAA GAA T A C C GGT T CA G CTAAAACTAAAGCGTGCTGGTTCTTCACTGGAAGCAT AGGTTGGTTGTTTTTTCCTGACAAATATGGAACG TTTAGAAGAAGTCATGGTAAAGTTGGGATTCT CAACTGATTCTTCTGAGCAGTTTATTTGAGAGGAG CCTGAGGAGGCAAGCATCCTAGTGAATGAAGTATTG CTTCTATATAATGGTGGGAGGAGATTTGGATCTTTG ATTGTGGGTGGTAGGGGAGCTGCAAGTAGCGTTG CTGGGTGGTTGGGGAAGGGGCGAAGACCATTAGGG TGGATGTTTGAGTGATACAAGGGAGAACGCAAAGG ACACGACGAAGCAACGAGTAGTGCTGGGGACTCGAG TTTTCGGATCTGAAGTTCGCGCTCTCTGCTCTATT CACC T GT T T T T GGT A A T T T T T CAAA GCTAGG TCCAAATTGCGGTGAAGATGAATTGAAGCAAATTC CATCTCAGTGAGTAGCTGTTATTCTTCTCATCTCCA TATTGCGTTCCGTCGTTTAGAATCAACGCTCGGCCG AGCATTTCAATTTGCCCGAAAAACATGGGCATGG CTAACGGAACACGATTGAAGA

LEFT PRIMER CCTCTGCTTAAATACTCCGA RIGHT PRIMER CAGACTGGCAGATACAATCA PRODUCT SIZE: 108

\section{G1_Unigene_BMK.782 p6 (GGCGGT)3 18417434}

GGACTCAGTGCTCTCGCGACTCAGATCTGAAG TCCAAACGACGCAAACGCTCTCACGGTATCCACT CATTCGAGGAGAGGAACATGGGTCGTGGAGTCAG CAGCGGAGGGGGAGAAAGCTCTTTGGGCTATCTG TTTGGAGGTGGAGAAGAAACTCAGAATCCCGTGGCG AAAAGGGGTACGGCTCCTCAGAACCAGGCGCCGG TTCCAACTGCTGAACCACCTGCTCAGAGGGCTACTG CTCCCGCACCACCTGCAGAAGTCAATAGGGAG ATTCCCGCTGGCGTACCTGGGAACACTTCAAACAAC T A T T T C C GA GC T G A T G GC C A G A A C T G C G G
CAACTTCCTCACAGATCGACCATCAACGAAAGTG CACTCA GCCCCCGGTGGTGGATCATCCCTAG ACTACCTGTTTGGAGGCGGTGGCGGTGGCGGTGG CAAGTGAAAGCCTCACGCCGCTGAAGTTGGAATCG TCAGTTTGGCCCTCGCGCACCTTTGTAACTTGG ACCCTCTGTAGATGACTTCTCCTCTGTAACATTC TAGCACTTCCTGCGGCGGGGATTTGTTGGGTTTAAG CTTTTACGCTGCTGAAACTTTCGAGTTAAGTTGG CCGATGATATAATCGTTCACAAATTGCACTACATG TTCTACAAGGATTTTTCGTTTTAATCGAAATTTG TACAAGTTTTTTAGATTCTGTTCGGAAGGAAAG AAGCACACGAGAAGCGTTTCTCCATGAAGTCG CTTAAAAAATCAATTGATGTTATCGAAACCCCG

LEFT PRIMER AACTATTTCCGAGCTGATGG RIGHT PRIMER AACTGACGATTCCAACTTCA PRODUCT SIZE: 172

\section{G1_Unigene_BMK.949 p6 (AGGGCA)3 18200217}

AAGCACAACGTGGAGATGAAGAACGGCAAGATCGTG TTCAAGGACATGGTGCGCGGAAACGACGATCCATTG CAAGGCCAGGGATCCGGTATCGATGCTTACTTGG ACGCCGTCAAGCAGGCTCCAGTGAGAGGCCAGAAG AACAAGTTGAAGTTTAAGAAGACCAAGGACGACG AAGAGTGGTCTGACTCTGAGGACGAGGGCAAGGG CAAGGGCAAGAAGGCCCTTCCAGCGCAAAAGGG CAAGT TCTCGAA GA GTGGTGGCGTGGGTAA G CCAAAGCAGAAGTTCAAGGCGAGAAAGAAGCTTTG AGGATGATGAAGGTGAGAAGAAGCTTTGAGAAGG ATGTTCAAGGCGAGAAGACGCTTTGAGAAGGATG ATCAAAAGGCTTGAGCAGGGCCGCGCCTGAAGTG TATATTAATG

LEFT PRIMER TATCGATGCTTACTTGGACG RIGHT PRIMER GCTTCTTCTCACCTTCATCA PRODUCT SIZE: 243

\section{G1_Unigene_BMK.1448 p2 (AG)11 22950971}

TTCATCGTTTGATTGTAATCGTGAAAATTTTCATTG TTTGCAGGAAAATATTGCGTTCTTCTATTCGTTTTAG TATAGTTAGATTGTTGAAATGATGCAGCAGCAGCAG CATAGGAGCAATCATGCCTGGCCAAAAGTGTAAG AAGCATGAAGGAAATTTGCAAACAAATTAAACTAAA CACACGGTTTGGCTACATATCAAAGAAAGAATACCG AGGTGCAAGTTGCAACAGACAGCAGTTGCAACATG CCAACATATACATTCAAAGTAAACAGAACACAGTTA CAGACAGGTTACAAGAACATCATGACCCAGAAG CCTGGAACATCCATCAGCTGATGATGTTCACCAACG AGGGGGCCCACCACCGCCACCACCGCCACCGCCAGG CCCACCACCACCGCCACCAGGTCCACCACCAC CAGGGCCCCCAGGTCCACCCCATCCACCAGGGCCAC CAGGTCCTCCACCAGGTCCGCCGCCGCCCCATCCAC CAGGTCCACCACCAGGTCCTCGGCCGAACATTGG CCCACAGCAGCGCTCAAACAGGCAGCAGCAG CACAGGAAATAAGCACAAGATGATATAAGGTTGCAT ACACCGCCACATAGGAAACCTAAGCAATCAAAA C A C G G T G G A C C T C C T C C C G G T C C T C C C G G CCCTCCTCCTGGTGGCATGGCTCTCTGATGACGATG ATGATCTGATCAATCTCTCTCACTCTCTCTTG ATCAAAAAAAGCCCTGAGGATATTTCTGCAG CTTCCTTTCTTAATGAACTTGCAACGCAGCTATTG AAGTTTGTCCGCTATATATACATGAAATTCTTGTAG AGAGAGAGCGAGAGAGAGCGAGAGAGAGCACG 
CTTAATGGCTAAGTATATATGCCTGAAACTAATG TCGGCTGTATAGTTTCATGTCGGCTGTATAATTAAG TTAACACTTACAGAGACACAGAGAGAGAGAGAGAG AGAGAGATGGAGGTGCTGCAAATACTTCACATG A A C A A A G G G A A C G G C G A G A C T A G T T A T G CTCAAAACTCTAAATTTCAGGAAAAAATATTATC C A T C G T A A A G C C A A T C G T T G A T G A A A C T G T A C A A A A A T T G T T G T GC T C A A A T A T A GCAC CAATTGGGAGCATGGGAATAGCGGATTTGGGTTG CTCATCTGGACCAAACGCCTTCCTAGTTATCTCTGAG ATAATTGATGCCATACATGCCACACATAGCGGCTCAT CATCATCCACAGAGTTTAGAGTGTTTCTAAACG ACCTCTTTAGCAACGACTTCAACACCATTTTTATG TCACTCCCGGCATTCTACAACCAACTAAACGAAG AAAAGGGTGCTGGACTCGGGTCTTTCTTTGTCTCCG CCACGCCTGGCTCGTTTTACGGCAGGTTGTTTCCAG CCAAGAGTTTGCACTTCGTGCACTCTTCTTCTAG TCTCCATTGGCTCTCTCAGGTCCCTCATGGCCTGG ATTTCCAGGCAGCTGGCAAAGCTTTGAACAAAGG AAAGATTTGGATTTCTAAGACCAGCCCTCAATGCG T T TTGGATGCA TA T TCATT TCAATT TCACAAGG ATTTTTCGTTATTTCTAATGTCCCGGGCTGAAGAAA TAATTGGTGGAGGACGGATGGTGTTGTCACTCA TAGGCAGGTCATCCTCTGACCCATCAACTCCAGAG AGCTGCTACCAATGGGATCTCCTTGCTCATGCAT TAATGACCATGGTTTCAGAGGGTCTTATTGAAGAGG AAAAGGTTGATTCCTTCAACCTTCCGTACTATG CCCCAAGTGAAGAGGAACTGAAGCTGGTGTTAG AAAAAGAAGGATCATTTATAATAGACCGCCTTG AAGCCTTTGAAATTGATTGGGATGGTGGTGCGG AAACAACCGTCGTAGCCAGTGGTCAGCAAGCGG CCAAGACCATAAGAGCTGTGGTTGAGTCGATG ATAGAATCTCATTTTGAAAAAAATATCATGGATG A TCTATTTCGTCGGTACGCCGAGCTCGTCGCCG ACTACTTATCAAAATCTAGAACCAAGTTCCTCA ATTTGTGTTATTCAGTCATTAGAAAGGACTGAG TTTTCTTAAACTTCCCAATCAAATTTTTCGTCAACAG ATTAAAGATCTGGTCTCATTGTCATATTTTTAG T T A T G T T G T T G G A T T T T G T T T T A G A A A A T G TTAAGGGGAGATCATATTTAATAAATTATGTTTG TACTACTTTTCTAATAAATGTGAAACCTACTTG AACAACCTTACTTGTATCGGAGGTTCTACTTCTAT TAAAAACATAGACGTGAAATCTACTTGAAGAACCC TACTTGATTTGTTTTACATGTAATATAATTACGGG CTTGGCCCCATTACTCATCCAGA

LEFT PRIMER TGAAACTAATGTCGGCTGTA RIGHT PRIMER CATGTGAAGTATTTGCAGCA PRODUCT SIZE: 115

\section{G1_Unigene_BMK.1461 p3 (TTG)7 2120002020}

TCTCTCTCTCTCTCTCTCTCTATACCAAGAGGAG TTGGTTTTGATGGCATTGGTCGCCGCCACCTTGGAAG T T C C A A A C C A T A C G T A A T T G A G G A C G A T G ACTCTCGGCACCC T C C C T G TAA T A T A A T T G T TCT TTCTGCACCCAAA T TCTCTC TCTACTCTG TTTTCTCСТCTCTGTCTCTCTGTGTAGACCAATAGG AGTTGATTTTGCTGGCATTGCTCGCCACCTTTGAAG TTCCAAACCTGACGCAATTGAAGGGAAATGACTCG CGGCATCCTACCGTTGGCAGCTTCGGATCTTCATAT CA T GGA T GGAAC T C GAAGA A A T T C A C A C T G ATATGTGCGCCGGTAGTGGCGGATTCAGTTGATCAG ATGCTGGTCCAAATGGGGAAGGCAAAGGAAGTTGG
CGCTGACCTCGTTGAACTTCGGGTCGATTTTTTG AAGAACT TCAGTCCAAGACAAGACCTCAGTG TATTGATCAAACAAAGTCCTTTGCCTACCCTTG TCACTTACAGGCCAGCATGGGAAGGCGGTCAATA TAAGGGTGACGACAAGCAGCGACAAGATGCACTG CGTGTAGCCATGGATTTGGGAGCAGATTTTATTG ACGTTGAACT TAAGGTAGCCGACGAGT TCTA CAATTCCAT TCAAGGGAGGAAGCCAGAAAAGG TCAAAATCATTGTTTCT TCACACAACTATGAG AAAACTCCGTCTGCCGAGGAAATTGGTGATCTTG TTGCAAGGATACAAGCTACGGGAGCTGACATAGTG A A GA TCGCA ACA A C T GGCT T A GACA TCACCG ACTCTGCACGTGTATTTCAAGTATTAGCGCATTCT CAAGTCCCAATGATAGGGCTTGTTATGGGCGAG AAGGGTTTGATCTCACGCGTACTTAGTGCAAAA TATGGTGCATTCCTCACTTTTGGTACTATAGAGG CGGGAGTAGTATCGGCTCCTGGGCAGCCCACCG TAAGAGATCTATTGGACTTGTACAACTTCAGACTTA TAGGGGCCGATACCAAAGTACATGGTGTGATAGG AAATCCTATCAGTCACAGCAAAATCCCTCATCTTTT CAATGCAGCATTCAGATCTATCAATTTCAATGG AATTTATTTGCCTTTGTTGGTTGATAACGTTGCG AATTTTATTGACACCTATAACTCCCCCGACTTTG TTGGATACAGTTATACAATTCCTCACAACGACG C T GGGA T T A A A T GC T G T GA T GA A A T C G A T C CAAATGCCAAGGAAATAGGAGCTATTAGTTGCATG ATCAGGAACCCGGCTGATGGGAGATTAAAAGG CTACAATGTGGATTGTCTAGGAGCAATTGCAG CAA T T GA GGAAGGACT TCAAGCA T T A A T GG TTCAAGTAATGGATCTGGTTCCCAGTTAGCCGG T A A A C T A T T T G T C G T C A T G G G A G C T G G T C G TACTGGAAAGGCACTTGCACATGGTGGAAAACAG AAAGGAGCAAGAGTGGTAGTTGCCGATCGCACA TATGACAAAGCCAAGGCACTTGCAAACAAAG TTGGAGGAGAAGCTATCACTCTTGCTGAATTGG AAAATTTCCATCCAGAGGAGGGAATGGTTCTTG CAAACGCCACA TCGGTTGGAATGAAGCCGAG AATTGATCAAACACTCTTATCCAAGAACGCTTTG AAGAACTATTCTTTGGTGTTTGATGCCATTCATA CACCTAA A T G G A C A G A C T C T T A C A A G A G CACAAGAGTCTGGAGCAGCCGTTGTTTCCGGG ACGGAAATGTTCCTCAATCAAGCATTTGTACAGG TTGAAAAGTTTTCTGGTTCACCTGCACCAAAG CAACTGATCAGGGATGTGTTGGCAAGAAATG CATGAGGAATGTGTCTCAAATCTCCCGTTCCAG CCAATCCTGTGCTTTTCCAGTTGTGGTTAACGGG AAGATT TAGTACAAAA TCGAGTGCAGTGGCG TTTCAAGATTCATAGAGTCGACTTCACATAATG AGATACGGCTTTGTTGTTGTTGTTGTTGTTGTAG CTATTAACTTACCATGTTGTACCAATAAAATTTT CAGTTTCACATATGGAGTGTCTTGTGATTCTGG TGAACATGAATT TCGAAACGTTTTGTACTTAGG TCATAAACAATTCATTAGACAAAAGGCGTGAATTG ATACAATTTCAAAATTTTAGGTGCCAATAAGAG CAAATTGAAACCTCGTGGCCTATTTTGAAAATCACCC CAAACAGGGAGGTGTGCAATGTAAGAGGCAACTGGG ATTAGGCTAAGCTACATAATGTGTCAGCCATAA CAATTTGGTTGACCCTTTTGGCGAG

\section{LEFT PRIMER AGTACAAAATCGAGTGCAGT} RIGHT PRIMER ACCAGAATCACAAGACACTC PRODUCT SIZE: 159 
21. G1_Unigene_BMK.1655 p6 (ATGGGA)3 18722739

GTGGTGGTGGTGGTGATGGTGATGGTGGAATCACA CAACCGGTGGGTGGCAGAGGAGTCACCGGATGCG ATGGCTCAGTTGGTGGAGGAGGAGGAGATGGATG ATTTGGTGGTGGACTACTTATACCTGTGCTTGTGAG TGGTGGTGGGGTTTTTGATGAATAATGCTTGTGTG TGGGTGAAGGAGGTGGTGTTGTATAATGTTCGTG TGTTGGTGGGGGTGGAGATGCATATGGTGATGG CAGTGGAGGTGGCGGAGGTGGTGAAGAAGATGG AGATTGTGGGGAAGGTGGAGGCGGTGATGAAGG TGGAGATTGTGGTGAAGGTGGAGGTGGAGGCGG TGGACTTGTGTAACTATGATAGCTTGGAGGTGG TGGGACGTTGGTTGAGTGCTGTTGAGGTGGGGG AGGCGTGTGGGGCTGATAATCGGGAGCTGCTGCG TGCTGTGGTGGCGGCGGCGGCGGCGAAGCATA ATtGTAATGATAGCCTGGAGGCAACGGCGGCAC CA T A T CA T G T T C A GA G T GA GG T G G T GGGGG C G G G G G T G G T C T T A G A T G G G T A C T T G G T G ACACСтCтTGGGAGGGTGGTGGTGGTGGTGGCGG CGTCAGAGTCCAATGACCATGACTACTGGATGG AGGGGGAGAGGGTGGAAGATGAGTCCTGGGCG ATACTCTTTGATTAGGTGGTGGCGGAGGTGAAGG TGGTGAATGGTAAGTGGGCCTTGTATGGGAATGGG AATGGGAGTGAGGAGGAGGTGTTGTATGAGGCG CTACAGGCGAAGGAGGAGGAGGAGAAGAAGAAGG CTGTGATGGTGGCGGCGGAGGATGTGATCTAGTCG AAGGCGA TGACTCTGAAGTCGGGGGTGGGGG TGGGGGTGTTGAAACCCGAGGCCTTCTTTGAGAAGG CGGCTTATTCCGAACAGGCGGAGAGGGTGTTGAG ACCCGTGGAGGCCTTTTATTAGAGCCGTCGCGG CCTCCTGGACTGCTGCCTCCGCCTCCACCGGGATTG CCGCCGCCGCCGCCTCCTCCAGGACTGCTGCTGCTG CATTTGAGCTTGGTGCAGTCGACGGGACGTGCAG CTGGGGAAGTACATTCCCTTGCAGATCTCTGATCCGG CTTGCCACGAATGCAGTTCTTGCGACCATGGATTA CATTATTTTTGTTAGAGATGGCAGCACAAGCAGGAG CCTCTCCGGTGAAATAGTTGAAGGAATAGGTAAAG TTCTGCAGCTGAGGCAGCTGGCAAACACTCGCCAG TATGACGCCGGTGAGTCTGTTGTGGGCGACGTCGAG CTGCTCCAAGCTCTTCATGTTTTTGCCGACGGTGG AGGGAAGAGGACCCTGAAGCTGGTTGAAGCTGACG T C C A A G A C G G T G A G A T T G G T GA G G A G G C C G ATCTGGGGCGGCAAGCAGCCGGTGAGGTTGTCGTTG AGGAGGATGATCTCGTTGAGGGTGTTGGCCATCTTTC CAA TGCTGGCGGGGA TGCAACCGCCGAGT T TG TTATTGGCCAACACCAGCACAGAAACCGGGGAGTTG CCGAGATTGTGGGGGATGCCGAATCGGAACCTGTTG TCGTTCAGGAAAACAGCGTCGAGATCCTTGTCGAAG AGCTGGGAGGGGACGGAGCCTTCGAACTCGTTGAAG CGGAGATCCAAGTACTTGAGGGAAGGCAGAGACAG CACTACTTTGGGGAAATCTCCGACAAAGCGGTTGTTG CTGAGGTCGAGCTCATGGAGCAGCTGCAGGCGGCG CAGGGTGCTTGGGACAACGCCACAGAAGCGATTGG AGTTGATGTGGAAGAGTGCAAGGTCCGAGAGGAGG CCAAGCTCCGGAGGGAGGTAGCCGGCAATGTCAGCG TGGTTGAGGTCAATGCCAGCCACCACGCGTCCTCCG CGGCCTCCAATTGAATTAGGAGCAGGAGCGCAG TATACGCCCATGTAGGAGCAGACGTCGGAGCCATTC CAGGTGGCGGTGAAGTTGAAGGGGTCGGAGAAG ATGGAGGACTTCCAAGCTTGGAGGGCAACGTAGG CTTGGCGAAGCCTTGGGTTCTCAAACTGGAGGAGG CTTGGATCTACTGCTTTGACAAGTTCATCAGTATCAG
TAACAGCAGTACTAGTACTGGCGGCTGCGCAGGCCAA CAAAACAAACAGCAGCAAGGAGGAGGAGGAGGAGG AGGA

\section{LEFT PRIMER CTGGGCGATACTCTTTGATT RIGHT PRIMER CTTCTTCTTCTCCTCCTCCT PRODUCT SIZE: 142}

\section{G1_Unigene_BMK.1726 p4 (AAGC)5 20766785}

GATTAATTAGAGGAATGTTTAAGAAAGATGACATG AAATTATTGAGTTTTAATTACAAGATAGAACAATCG T G T A A G T G T T G T C T A C A A C G A T G A C G A C G CСTCATCTTCCCGTTCTCTCCTCCGTTGGTCTTG TTCTTGTACTCCTTTGAATTCGTGCTGCCGACGCTG TCGCTCCTTTGATTTGATCGACTTCTCATTCTTCTG TCATTAAGTAGGAGAGACAAACATTATTACAATG CTTAATGCTTATGCTATTTGCATGCGGTGCGATGCG ATTTGAGATTACAATCTTATATATACTTGGCTCGG T T G G T T G G T T T G A A A A G T C G G T C T G A T G CTCTTCTATCCACTCATCATGCTCTAATTGAAAT TTCTTTTTCGCTTTAAGCATTTTTTGGATGCCAAAG ATGATTTTGGAAATGAACAGACACGTAGCTCAAG TAGTTATCAGCATTTACCCTGCCTTCCCCAATATTC CAAGTATCCGGAATTAACGTTGGGGAAGGTGAAAGG AGAAACTTCTGCATTGCAACTGCAATGCCAAGTG TAGGATTCTATTTTGGGCCGGAAGAGAATAACTCC TATTGTCCTCCAAGCGAATCAGTAAAATAAGGTG AAACAAAGAGGAATCCGATGCTTATCTTTTACACG TTTTCGCTTTGAGGCCACCCGCCCCGTGCACCA CAAAA A CA T GACA GCACGT T GACGT T GGATG CACCCGTTGCCAATGCCAGTGCCTAGGACACCG TTCTACTTACGGAGTAAGCAAGCAAGCAAGCAAG CAACCAAGTTTGTCGAAAGAAGCATGCATCAGTG ATTCATTCCCTACCAAGTTATCATCTACAGAAAG CTATTAACAAGTTTCATCAGATCATAACCACCATCGG TGATCAATTTCAGACTCCGCCAATGGAGTTTGAAG AATTTGCAATCCCGAAAACGTACTTTCAATTTATG AGGACACTGATACTGAGGAAAAAGTTACATAATAC TAACATGATTTTGAAATTCAAATTTCTTGCTTTAAG ACAATAAACAACAGAAGAAGCACGTATAACCAACG CATGATGACAAGATCACATTAACAAGAGAGGCTAC CAACTTGCAATATTAACAACGAAAAGCAACAACAAG CAGTAATATCAGATTCACCCAATAGATTTAAATT CATGATTTCAAAGAGAAGAATCAGAATACCAATG TATCGTAACAGACACTACCAAACAGGA

LEFT PRIMER GTGAAACAAAGAGGAATCCG RIGHT PRIMER GAATCACTGATGCATGCTTC PRODUCT SIZE: 207

\section{G1_Unigene_BMK.1812 p3 (CGT)6 1811501167}

AGAAAATGAAGATACACAAAGTGGAACTTTAG TTATCTGATGATTTTAAAATATGTCTAACCAAA CATTTTAAGCTAACTTTTCTTGAAAAGCATTCTAAG AAGAAAGCAAATATGTAAAGTACTTTCAAGTCG CTTGTTGATTTACCAACAAATGCCTGCAATCCAG A T T T G T G T T G G T T T A A A C C A C C C T T T G T C G CTCTAAAACTTTCTTCGACATTCATTGATAAATTA CAATATTTGAAGTACCATCCTAACCTAGATTGTAG AGCACTTCATCTGCCCCCACAACTAGGACAATAG TGGTTACGAATGTTCTCTAAAACGTCTCCACATCTG TAGCAGAAAGCAGTTCTGCACCTGCACATCATG AACATGCAACCCACCGATTTTTCCACATAGAACCTA 
CATTTGGGGCATCTTCTCCATTGTTGCTTCTGCG CAAGGTTCTTCAACATGATATCCTCCCTTTCCСTCT CATCCTTATTCAACTTTAGAAACTCCTCGCATTCG ATCCCCTCGTGCCAAGGAGCCTTACACTGAGCACAG AACAT TC TCCAACAATT TGGACACGCTGATTG CCTCACAACCTCTTTCCCATCATTAATCAACATTG CAGAGCAATCCTTATAGGGGCAGTAAAATTTCTCTG A C C C A A G A A T C A C A GCC T C GCACA T A GCA G T T C C C CAGCGA TCAAACACCTCCGGAGGCAG AATCGGCCGGCAATACTCGGGCTCCAGCAGCCCTA TACAATCAGGAACAGGGCACCTAATGCTGGTAATG TTCTCTTGGAGCTTGGAGGCCACGTACTTAACCATA CACTCAGTACAATAACCATGGCTGCAGTTTTCG ATCCGAAACAACTCGATTCCCGACTTGTGATCGACG CAGATTTCACAAACGAAATCTGGGACACTTTTAG A A T T T GA C GA T T GC C C A G A T T C A G T C A C G G AAAAATCAGAAAAGGTTTTTTTTCTTCGCTTTCCG A A G G G A G T G T T G G A T G G T T T A A A C C T A A G AAT TCTGAGCTCGTCGTCACCGTCGTAGAAG TCTAGGGTTTCTTCAGAGAGGTCGATAAAGTTG CTTGCTGCGGTGGGCTTGGAAGTTGATTTGAGCAG AGAAGCCGTGATCGATCGAAGGTCTCCGTCGCCG CCGTCGTCGTCGTCGTCGTCATCGACGTTGATGGGG ATGGTTGAGGGATTCCGCGAACTGGGTGTGAAG CTGACAGCGTCGTCTTCCATGTCGAAATTGGGGATG TGGGAGTGAGACGACAGTGGGTTCTCCATCTTTAG CTTTCTTTCTTCTTCTCATTGGCTGTCAGCGTCACTG A C A G A G A C T G A C G C T T T T G G A T G C T C C G CCTCTCTCCAATTTCACTTTCCTCCCCTTCGTACTG TACACGCTATTTCGG

LEFT PRIMER TAAGAATTCTGAGCTCGTCG RIGHT PRIMER AATTTCGACATGGAAGACGA PRODUCT SIZE: 235

\section{G1_Unigene_BMK.2010 p3 (GCA)6 18783800}

TTAAAACCCATATTCCGAATAGCCGGCCAAGAG CCACGGCGGCGACGCCCCACAAGTATACTTTATCCTG ACCCACTTACCGAATTCGCAGAACCTGCAAGAG ACCCAGAAGTTCAGGGCTGAGCATTCCAGGAGGCTG T T G A A G G A G A A G G A G A C T T T T G G G C C T G ACCTCCACCGTGTGATAGGCGTCTGTGCAGAGG TAATCAGTGACTTCTTGCAAAAGGAGTATGGAGG ACCTGAGCCTTGCACTGATATATTGGTTGCTCTG AAAGAGAAGGAACTACCAGGAGCACCTTTGGCTG CAAGAACATCTCTGTTATGGGCTCAAAGCTACCTCG ATCGAGACTGGGAAGTTTGGAACTCGAAAACACC CAAATGATGTATCATTTTTGTAATCCACACAAATT TAATTTTTGGTTTTGTATTTCTCCAGATCATTTA T A T T T C A A A C A C A C A A A A T G G G G A G A G ACCCATTGGCTACCAGGAAACTCACACTTCTAATA CAATGTCAGATTCAGTGCTTTTTTTCTGTGTGTTG CTGTACAGATGGATTATTGCTTTCAACATGGGATG A A T T T T T GGGGG T T GGGGGGTGA T G T GA T T G ACATTGGATTAGAAAACTTGGTACCCGACTCCATG AATTTCGGGGTTTCGATCCATTTTTGCACTCCATTG TCTACTCTGCATTCTGTTCGCTATCGAAGTTTGG AAACCGCTGATCAGTTTACGCCCTCGGAGATG CTCTGCAACGAGGGGCGCCAAGACTTGGACCTCAG TGCGGCAGCAGCAGCAGCAGCATTCGCTGATGATG TCCTTGATCGGTGCCTTTCGCGTTTTGAGGCATG C T T G C T A T T C T C C T T C T C A T C A G A T T T
CACT T C T T C T GC T GCC T C T T C GCAAC C C GA G CAACCCGAAT TCTCT T TCCCT T GCCCCGT T T G C A A C T T C T C G A G C A A T T C G A G C T G C T G TTTCTTCTTTTTCGCCTTCAAAACCTTCATAACC TCTTGAGTAGTGACCAGCCGGTAGGCATGGCCGAG AGCGAGAGTTTCGTTTGGCCGAAGAAGCTTGACG C G A G T G A A G C G C A C C G C T T T G C C G T G CTCCTCCTTGTGATTCTCTGCTACTTGTTCGTG A TCT TGT TCGGAAACCGGCAACGGAA TGATG AGGGAAACGTAGTGACCGGGGTTCAACCTCAT C A C C T C A C T C G C A C T T A T A G G C C A A T A CATCCTCTCTAGCTTCCCACTCGGATGCTGTATCAC C A G A G C T G C T G C A T C A A T G G C T T G A C A G TTCCCCATCTCTCTCTCTAGAAACTTACGAAAG AAGAAGACGACGACGACGACGAAGATAGAGAG ATAAGGAGGAAGAAGAAGAAATAGAAGAGGAAG AAGAAAGAAGTCACT T T C T GT A CA GC T C T C C TCCCATTTGTTCACAACCCACAAACCCAAAGATA TAAGCAAAGGTTTACCATATTT

\section{LEFT PRIMER TCTACTCTGCATTCTGTTCG} RIGHT PRIMER GCAGCAGAAGAAGTGAAATC PRODUCT SIZE: 212

\section{G1_Unigene_BMK.2117 p6 (TGCTGA)3 18465482}

AGCGAGTCGAGGCCCGAGGGTTTATTGACTCGAGAG AGAATCGGGAGACACCGTGGAGACCAAATTAGGG TTTTAATTTGGGTGTGAGCAGGACCAACGAAGAAGG AAGAAGCAATGGAGGGTCTCGCAGAAGGAGTG AACAACTTGCGAATAACAGACGCATCACACAACAAG AAGAAGAATCGCATCCAAGTCTCCAACACCAAAAAG CССCTCTTCTTCTACGTCAATCTCGCCAAGAGG TACATGCAGCACAACAATGAGGTGGAGCTCTCCG CTCTTGGAATGGCTATTGCCACAGTTGTTACCGTTG CTGAGATTCTGAAGAACAGTGGATTGGCTTTGGAG AAGAGAATCATGACTTCTACAATTGACATGAGGG AAGGTGCAGGAGGGCGGCCCATTCAAAAGGCAAAG ATTGAAATACTGCTTGGAAAGTCAGAGAAGTTCGACG AGATAATGGCTGCTGATGCTGATGCTGATGCTGCTG CTGCGGCTGAGGAAGGCATTGAGTATGAGTAGAGTTG AAATGTGTTGTTTCTGATATCTAGCTTGGTGCATG CTCTAGTAGGTTTCTTTAAAACCAGTGGATGTCTTG ACTTTTGTTACTAGATTCGTCTCTCTAGTGGCATTTTG TTTAGCTGCGGTAAAATTTCTTTTAGGGATTGTTG T A G G A A A G C G T T A T C T C T G C A A C C A A A A G ATTAAAAGTTATTCCTATGTTAATTATCTAAGCCAA C A T G T G T G T T A G G A T G T T A T GA A T T T A T T G CTTCTTTCTGCCGGAGCATGGAATTAATTTAGCTTTTG CGATTTCAATGTAATGCACTGATAACACGCTATTGG TCGGGAACTAAATCAAATGATATAATCAATTTACA

LEFT PRIMER TGGAAAGTCAGAGAAGTTCG RIGHT PRIMER AAAATGCCACTAGAGAGACG PRODUCT SIZE: 204

\section{G1_Unigene_BMK.2121 p6 (GCTTGC)3 18506523}

GAGGCCAAGTATATGAGTGTTGAATGACCTGAAT T T C A T C GA A A C A G A G A A T T T GA T C G T G C A A CAAAATCCGTTCCAATTTGGTAAACAATAACTTGTCG CTTGAAATGGATAGTGCACCAAGGTGGCAAGTTTCG ATCTAAAACTCATTCGATTTCCAATTAGATAAAAAAG CATTACAATGGGATGGGGTTGAGTAGAGCGTTG 
ACAAGTTCTCTCATAAGCTAGACACAAGTAATC TACTCTAGCATTGCATCCACTGAAGCTTCGGGGGTGG CGGCAGCA GCA GCA GCA GCCGCCTTAGAAG ATTTCCCTTTCTTCTTACCTTTGCCTTTTAGTTGTTTG ATCTTGAGACTCTTCTTCTTCATGGGAAACTTTGAAG AACAAGTCTTGGTGTCTTTAGATTCTTCTCCATCAG CATCGGCAGCAGCCATTTCGACATCTTGCATAGTTA TAAGTCCCTTCTTCACAGCCTCGTTCTGATCTCGGCG CCATTTTTTCAAGAGCTTGCGCTTGCGCTTGCCGGAG A T GGAGA GGGGT T GA GGCTTGTTGTGGA G C T T C C C G G T G G T T G G G T C G C C G T G A A C C G ATCTCTTCTTCTCCGCTTTCTGCGCTCTCTTCCTC TTCTGCACCTCATTGAACTTCGCCATCTCTCC TCTCTCTCACTAAACCCTAGCCACCGCCAACTG CTTCAACTTGAAAGGAAATTATTGTCATGGTTG TTTTACGAGTTACGAG

LEFT PRIMER CCATTTCGACATCTTGCATAG RIGHT PRIMER AAGTTCAATGAGGTGCAGAA PRODUCT SIZE: 211

\section{G1_Unigene_BMK.2184 p3 (GAG)6 18340357}

TGCCAGCATCTTCATCCTCTCTTCTATATCGTCACGG ACGGATAAGAGCCATGTGGGTTTCCGATTCATGG TGGACTTGTTGCTGCTGATAAAACTACTTGGACTTGG AGGAGCCGACGACACAACTGAAGAAGGAGGATTTG ATGAAGCTGCTGATGTTGTTGTTTGTTTCTTCACCG CTGTCGATCCCATCGATATGTCTCAACCCTCCTAC TAGGCTACAATATACTTGTATCTAATCCCTCTG ACTCGTGTTTCTGAGAGAAGAGAGCAGAAGCAGCAG CACCACACAAACT T AA T T C T T T GCCTTGTAG TACAAGACATTAAAGCGCAGATCTCGAGGAGGAGG AGGAGGAGCAATGAATTCGAAAAATAATAATG TTCAACGAGCATCAACACTGTTCATTTCACATATGTG TATGTATGCATGTGGGTTTGGCTTTGGAA

LEFT PRIMER TGTCTCAACCCTCCTACTAG RIGHT PRIMER GTTGATGCTCGTTGAACATT PRODUCT SIZE: 200

\section{G1_Unigene_BMK.2519 p3 (GAG)6 18228245}

ACATCACCCAAGATTTGGTCTATGCCTCTGTACTTCT CACCAAAAAGAAAACACAAGCTGAACTAACAAGATG AACAAAAAAAAGACATGAACAGAAGAAGGAGAAG AAGCCTCGGAGGCATAAAAGTAGTAGTAAGGATGTG AATTGTTATTCGCCACTCCTGCTCCTGTTGTTGCTG TTGTTGGCACTGGACCTGCAGGAGGCATGCCGTAG AAGTACGTAAACCGAGGAGGAGGAGGAGGAGCG CCTTTCGAAGGATATGATGGGGGGCATTGTTTTGG CCGTGAGGGAGGTGGCGAAGGTGATTGTTTTGGTGG CGAGGGAGGTGGAGAAATGTAGTAATATTGAGG TGGAGAGTGGTGTGCTGGTGGCGGTGATTGTAG AGGTGATGGCGGTGGACCTGAAGGCTCGAGTTG A GGAGGAGGAGGTGGTGAACATATGACTGG ACATGTAGTGCAGTCACTGATGCATATTAAACCAG TCTCAGGATCCACCGTTTGATCTCCATATGTG AACCTGGAAAACACGATCATCCCGAAAATCGCTG CCATCAAGGACCGGGGAATCCATTTCCCTACCATG AATCTCAAAGCTCTGGAGTTAGCCATCTGACAG AAGCATAACCTTGAAAACTGACCAAGTTGTGAGG TAGCCTTCAGAAAATTTTGTTCTTCTTATCCG CCTTTTTATACAAACGTA
LEFT PRIMER AATTGTTATTCGCCACTCCT RIGHT PRIMER CCACCAAAACAATCACCTTC PRODUCT SIZE: 175

\section{G1_Unigene_BMK.2540 p3 (CGA)6 185774}

CTCСTTCTTTCCСТCTCTCTGCACAAAGACGACGACG ACAAAAAACAGACAGAGTCCGACGACGACGACGACG ATGATCAAAGGGCTTGTTCACTTCGTGTTCGCAG TCTACTTGGTTTCTCTACCGTCGGGTATCCAACTG CCCGTGGACGGGCGGGTTCTCTTGCTGAAGAAACCCG ACCCGGAAAATGCTGCCGCCACTGCTCGTTGGTTGG TCGCTCAGAATTCCTGGGGCGTCTTGAATACTATCT CAAGTGAATTGGGAGGAGCACCCTTCGGGAATGTGG TTTCATTTAGCGATGGAGAACCTGGAAAAGGCAGTGG TATCCCTTACTTCTACTTGACAACTCTTGATCCGACTG CAAGAAATGCACTGGAAGATCCAAGAGCTTCGTTG ACAATTAGTGAGCATCCTGTTGGAACCTGTGGCAA TAGAGACCCCGAGAATCCCGCTTGTGCAAAGCTTA CACTTAATGGGAAGCTGAAGCTTGCGGATCCCAAAG AAACAGAGTTTGCTATAAGTGCCTTGTTCACAAAA CATCCGGAGATGAAGGACTGGCCCAAGGATCA CAACTTCCAGTTCTTCAAATTAGACATCGAGAATATC TTTCTGATCGATTGGTTTGGCGGTCCAAAACCTCTTA CAGTCGATCAGTACCTTCATCCCAAAACGACAAAG AACGCCTTGATTCGAGGATTACTCGCGATCTCACTG TAATTCCTCTTGTATGTTCATGAGCTGGATGGATCCG CATTCGAATTGTGCATAATAACTCTGTTGTAAATCA CAGGAGACAATTGTAATTATATATAATCATCCCAATG TAAGTTCTGGAAGTCGTTCACGCAAATGCGCAG ACCAATTATCCGATTGAACGTTTGGAGATAA TAATTTGTCTGTACAAGGAACTGTATTTTTCTTTCTG CTTTCTGTGGTTTAA GCTTAAAATGTAACTCG CCCTTTGTAATGATGATTTGTGGTTGATTTAAAATGAG TTGTCCCGATTTCTCG

\section{LEFT PRIMER CTCCTTCTTTCCCTCTCTCT} RIGHT PRIMER GATAGTATTCAAGACGCCCC PRODUCT SIZE: 250

\section{G1_Unigene_BMK.3842 p5 (TCGGT) 315515529}

GGTTAGAGTTGAGGAACCATTGCTCAAATTGAG TTAAAGTTGAGGAACCATTTCTCCAGTTGGATTAAAG TTGAGGGACCAATGGTAATAGATTTTTAGTTGAGGG ATCATAGCTGCACATTTTGATGAGTTGAGGAAT TAATGGTAATGAAATTTTAGTTGAGACACCATTGATC CAATTGAGTTAAAGTTAAGGAACTATTGCTACAATT TACTCAATAAGAAAATAGAGAAAGTGGGAAGATGG TCATTTACAAGATCATATAAACCAATCTTATGCTAT TAACAACCTCAATTGCAAAGAAAAATGATGACCA TATCCATTCAAAAAGCAAACAAAAAGAGAAG CTCTTCCAAATTCCAAAGCCTTCCAAAACTTTCCTC TATAACCAATGAAATATCCTCCCTAATTGAACCAAG T A T G C T T C T T G A A A A A G T T C T A A G C T T A G TTTCAAACTTGGCAACCAAGCTTCACAAAAATGAG TATACATTCATAAAGTGTAAAGGATTCGAGTCGG TTCGGTTCGGTTTTTTTTTCAATCTTTCAATTTCGG TTAGGTGTTCGGTCCACTTTTTCGGTTTTTTTAACC CACCCCTAGCTCTCTTGAACTAGTTACACGTGTG CAGGCTGCTGCTACTTGGCGCT

LEFT PRIMER TTCCAAATTCCAAAGCCTTC RIGHT PRIMER CGAAAAAGTGGACCGAACAC PRODUCT SIZE: 227 
31. G1_Unigene_BMK.4019 p5 (CCAAT)3 15148162

GAGAACCTTCCCGGCCTTTCGCGTCTGCCCACCCATT CAAAAACCCTAGCAAAAACCTTAGAGAGCAGCAC CAACGTTTATGTATCCGTTAGTATGCCCTAGATTC CAAAACACTCGATAAAAACCCTCGTGAGAACCCC CAAATCACCAATCCAATCCAATCCAGTCGAATC CATCTATACTCTCAATGGAGCTGGTGGTGGCCTCG TCG T C T G T C GA A G C C GGCA T C GGC T G T T GGG ACCTCCTCAACGGCGCCGAGCAGCTCCGCTACAAG CTCTGCGCTTCTCCGCCTCATGGCCTCATTTGCG TCGGCGAGCGATTCCTCGCCTCCTCTCAGCTCCGTG AGCCTTCCGCCTCCTCTGGCTCTGTCCAGTACTGG TCTTGGTCCAAGCCGCAGGTTGATGTGAAGAG CTTCCCAGAAGAACCGATCAATCCGCTCGCTGCG AACAGCGAAGGGACCTACATTGCTGGCGGTGG CTCATCGGGGAATATCTACTTGTGGGAGGTTAA TACTGGTAGACTGCTTAAGAAATGGCATGCTCAC TACAGAGGTGTTAGTTGCTTGGCATTTTCGGATG ATGGCTCCCTGCTGATTTCGGGGTCAGAGGATGG AGGCATTAGAGTTTGGTCTCTTATAGGATTATTCG ATGATTATCAAAGTCAACAGGAAAGCCATCTG TACATGCATAATTTTACGGAGCACACTCTTTGTG TAACGGA T GT T G T C A CAGGATA T GGTGGATG CAAAGCCATTATCGTGTCAGCATCACAGGATCG CACATGTAAGGTTTGGAG

LEFT PRIMER GAGAGCAGCACCAACGTTTA RIGHT PRIMER AGAAGCGCAGAGCTTGTAGC PRODUCT SIZE: 226

\section{G1_Unigene_BMK.4067 p6 (CGGAGG)4 24436459}

AAAGAAAAACCAAAGCAAAAGAATAATCAAACG TCAGAGAAATCAAGGTCTACCAATATAAACAGCAA CAATAACATTAATCATACGCAACCTCGTCGCTCTAG ATCGCGAGCTGTATTGTCGGCATCGCCGTCCACGGCG TCGTATTGTCAGATTATCAGGCAATGGATGCGG ATAAGCGGAAGCGCGACGACGGAGGAGAATTGG AGGGGAAGAGAGCGAAAGCAGTCGAGGAAGATCG TAACAGAGATCAGGAGACGGTAACGGAGGAGGAGG TGGAGGAGTTCTTTGCGATACTCAGGCGGATACATG TGGCAGCCAAGCACTTGGGCAGAACGGCGTCCATGG AATGGAGGCCCTCATTCCAAGTTGAAGATTTTGAGG AAGATAACGAGTCCCGTATGACAAAAGACCCAAA AAAGCAGGAGAAACAAACGGAGGCGGAGGCGGAGG CGGAGGAGGAAGAAGAGCCGTACTTGAGCTTGAGGG CCTTGAGCCTGGCATCGACGTCGGCGACGGAGG ACTCTAGGGTTTTGGACTTGGGGTTCGAAGAAGCGG TTTGCGGTGGCTTGTAGTTGTCCTCTGCGTCGTCG T A T T G G T C T T C G T C C T C C G A T T G C T G T T C G TCCTCCTCCTCCGACTCGTAGTTGTCGGAGTCGATG ATGTGATCCTCCCGGCTCTGAGCGGTTCCCATGG ATTTTTGTCGGAGAGATGGAGAAATCGAGAAATG CGAAAGCTTTTAGAGGTTATGATTTTGTACGAGTG?

LEFT PRIMER CCCTCATTCCAAGTTGAAGA RIGHT PRIMER CCAAGTCCAAAACCCTAGAG PRODUCT SIZE: 187

\section{G1_Unigene_BMK.4181 p3 (TTC)6 18358375} GCAGATTCTCATTTCAATAATTGAATCAAATTCTA TAGTATGTACAAAGATTAAAATCTGTTACATG AACAATTTAACAATCTGCTCAAATCCTCAAAACTTCT TGAACTAATTACTTGATATCGAAATTCGTTTAAG
TTTAGTAACTACGAGTTACTGAGGCTGCTGCCATGTG CT T T TA T GGACGA T GA GGA T TGAA TACTGACG CCACGGAATTGGCTAATCTCGCCACTATTCTCTTCTT CACCTGACCCCTCT TGGGAATCGGCCGGCCAG AAAATCTCCTCGAGAAATCTCTGCCGTTCATCATCG CTCTCCCGGCGGAAGAATTGGATGTGGAACCTTC C A T C T C T C T A G T T C T T C T T C T T C T T C T T C T G TAAAACCTTCTGAATTCTGTATGTGGTTTTCTGAAG TTTTGATGCAAAATACAAGTTGGTTTTGCTCAATG CTCTTTCCAGAACCCACTGCTTTCTTATATACCAC TCAG

LEFT PRIMER AAATCTCTGCCGTTCATCAT RIGHT PRIMER GGTTCTGGAAAGAGCATTGA PRODUCT SIZE: 172

\section{G1_Unigene_BMK.4297 p6 (AGAGAA)3 18101118} CGAATTCCGTTGCTGTCGCTTCTCTCTCTGTTCTACA TAGAATAGCCGTCAATCCGTTGCTCATAAGTCA CAGCCTCGAAAGACAGAGAGACAGACAGTGAGAG A A A G A GA A GA GA A A G T A T A A A T T C A G AACTTGGCATTTTAACGGTCAAACATTAGCTTAAA CAGCTCATTAAGGCAGAAAGAGGAGAGAGGGAG AGAGAGCTTTGGTGAGAGCTTTGGTGATTCATAG TAGCTGGACATATATTCCGGCAATGTCGTTCGCCGG TTGCTGGAGGCTTCTGCATTCTTCCCTGCTGGTG ATTCTGGTCGTTTCGGCGTTACAGATTTGGGTCTG CTGCGATTGCAGGGCAGGGGCAATACGGGTATTCC CAGGAAATACAGATAATGTCATATCAAAGGTG CAGGTGGAAGCAGAGAGGAACACGATGGAGAAG A A G A G C A A G A T A A C A C A G A A G C C C T G TTCCAAAAGTACT TCGGCGGAAGCACT TCCAG TTCCAACTTCAACAAAACAGAAAAAGGATATGAAG A A T C C A A A A G A A G A G T GCCA G T T GCC CA G ATAAACTCCACAACTAGTCAAATTATTCAAATTG CCTTTTTTCTTAGGAAATATTTCTTGAATTAAAAC CAAAATACCCACGTTTTTCTCATATCGTTTGTCAAG TTCTATTCATTATGTTTTGGTAATTCATGAAGTGAG AAAACCGGGGGTATTTTGGATTAATGGGTAATTAATC TAGTTTTATAATTTCTTGAGATTGATGGATCATGAAT CAAGATTTGTACAGAAAAGTTGAGAAATTTGTACGAG C A T T T C T T T G A T A C A T A G T G A T G T G A T A G AATCTCCTTA

LEFT PRIMER TCACAGCCTCGAAAGACAGA RIGHT PRIMER ATCACCAGCAGGGAAGAATG PRODUCT SIZE: 240

35. G1_Unigene_BMK.4719 p3 (TCG)6 1899116

GGAACCGCTTCATTCATTTTTGTTCTCGCTAATTA CATGGGCGTTCTTTGCCCGATCTGCAATACCATAATC CAAGGTATTTCTACATCTTCCGTTCATCGTCGTCG T C G T C G T C G T C A T C A C C A T C A G T A G T A T G TCTCСTCTACTATTGTAGCTAGACTAGATAGCACTAG AACCTCCCTTTTATGTTGTTGTTTCCATCGTATATAT TATTTTCTTGCTAATTAATTATGAGTCTTATAAG C C T G T A C C A A G C T A A C T T G G C T T T T T A A G TTCAAATCGCTTTCCTCATATTCATGTATGATG AATAATATGATGTATTAGAGTATGTTAGGCTCAAG

LEFT PRIMER CCCGATCTGCAATACCATAA RIGHT PRIMER TGAGGAAAGCGATTTGAACT PRODUCT SIZE: 241 
36. G1_Unigene_BMK.5242 c (ACATCT)3(ACATCA)3 36203238 GGAAGAGATATCAAGTTTACGTGAGGAATTAGATAC CATTTCTAAATCACTGTCTGTTTCTGACATTGGG CATCTATCTTCTCACGGGTCCTTGGAAGTGGACGAAG AGAGTAATAATCATAAGAAGGGCGAGTCACACCA CAAGTTTTTGAACAATCTTAAATCATCGCCGTCAT TATCGTTGATATCTTCATCAAACTCAACATCTACATCT ACATCTACATCAACATCAACATCATCATCTCTTTGGG AAGTGGATGTCAACCGGCTTATGCATATGTCAACAG ATGAATTGAAAAGCTATTTCACTAAATTGAAGAG AAGTCATGAGTCTGAAGTGCAAGTTTTGACTGAG CAAATTTTCAGCCTGAGGAGAGAATTTTTTAGAG AAAGGGGTTCCTCTTTGCCATCGAAGAAAATCAAGG AGTTTGACATGTGGCGTAGGAGAATCTCCGAGG TTATTACCAAATTAGATGAAGTTCTTGTTGAAAAGG AGGAAGTTGCTACATGTGGTAATAATGAAGAAAG TCTTAGCAGTTTGA

\section{LEFT PRIMER TCTTAAATCATCGCCGTCAT} RIGHT PRIMER AACTTGCACTTCAGACTCAT PRODUCT SIZE: 190

\section{G1_Unigene_BMK.5607 p6 (AGGTCA)4 2413491372}

GAGAGAGCACTGAGCGAGCGCGCCTGTTTGGCAACG ACGCGACGGCCATTCCACCTGTATTTACCGCTCTAA CACGGGGAGGGGCGTCCAGATGTCTTTGCATATTGG CAATCTATCAGCTCACACTCGCGGAGATGATCTTAAA CATGTCTTTTGGAGGTTTGGACGATGTAACTTGCGG CTGAGGGACAGATATGGGTTTGTTGAATATGATTTTG TGCAGGATGCTAAGAAAGCGTTGAGAGCATTA CAGGGTAGGAAGATCTGTGGGGAGCCTTTAACAC TAACA T GGTCAAGAAAACAGCCTAAACCT T T T CACGGAAATCCAAGAGGTGTGAGCTCCTATGTGCTA CAACATGAGAGAAGAATGAATGAAAATGATTGGCG AGATTACGAATTGGACAATGAGCAACCAGATAGTG ATGGTAAAATGCTTAATTCTGTGAACATGCGTGATG ATGACAGAGGTGATCATCGAGGAGATATTCAAG ATTATAATGGAGATCGTCATGATTTTAGGGAAGG TCTTCCAGATGATATTGGTGGAGCTGTGCCCAAG C TGGTGGACACTGGCAGGTGGGGTGAACAAG TTCATGACCCATCAAACGCCAATGGGGTGGAATTTG ACCGATATGAACCTCACCAAGATCATGATAGGAAA TATGAAGATGAAAACTGTCGGATGGCATATTCTGG TGGTGGTTTTGCTCCACGAAGTTCCCAAGAGAATG TGAGGAATCAGAGAATGAATTATACTACTTTGAAC CATTCCAGTGATATGCGGAGTACTTGCTTTAGTTG T GGGGCA T C A G G T C A C A A GA T C C GA A A T G TCCCAGGAAATATTCTTCTAGAAGAAAGCTCA CAAGGTTTGATAATAGGCAAGATGATGCCGCAT ATGAAAGAAGTAGGGGTGAAGCTGAGCTAGAAAGG ATGGGATCTAGGTCCATGGGAGAAATGCAGTTG ACTGAGGGTACTGCATCAAGGTACATGAATGGTGG AATGGCAAGTGGTTCAGGAAAGCATAAAATGGTG ACAGACAATGTGAGCTGCCCTACAGGAAATGAAAA CAATATTACAGAAATAAAGGACCGTGAAGGAAA T A A G A G A A T A G A A G G G A A G G T G A A T C G CCAAAAAGGCACAGTGGTAAGAAAGCAAGGAGG TCAGTTTCATCATCACCTCATTCTCGTTATGCTG CATCCAGACTCAGATCATGCTCAACTTCTCAATCG TCAAAGTCTGTGACAAGGTCTGGTTCCCAATCTAGG TCAAACTCAGCATCTAGGGGAGAACACCACTCTG TA TC T TC T GA T T CAAGACTCCGT TCAACA TCA
A AAAGTT T GAAGT T TAGGTCAAGGTCAAGG TCAAGGTCAAGCTCCTCTATATCCTTGTCTGTAT CACTTAGTCCGTCACCATCCTCTTCAAATAAGGCG CA T ACCAA TCT T A A A GC T C T GCT GG T A C T C C CAAATCCAAGGAGATTCAGGTTAAACAGAGACAG CAAGTTGAAGGTGATGCCTGCATCGAGAAGGCTG ATGATGAAAATACAATGGATGCGATAAAGAGCAG TCATGCAGTA TCAAGTT T TGACGCAGAAGATG ACATGAAAAATGATCATCATTTTCAGATGAATGGTG ATGGTGAAAACCATACTATGTCCAGGTCAATTCATG AAGTAACAAATCCGGGCACACCTCTGCCAGAGG TAGGCTCTCATACTACTGGGAGTTTGTCCCCAG AGGGTTTGAGTGATTTTCAGGATCAACAAAACTC TAATGCACTGGTGATTGGACATGTACCATCTCAAT CAAAGGAGCCAGCTTCAGAAACCCCTATCTACCG CACCACTGGTCGTTCAACAAGCATATCCTCAGATG AGTTGTGTATGGTTTTGAAGCACTATGACCTGGAAC TACCAGAAGAAAGTGAAAGGCATTTACCCTTCG AGGGCTACTTTGGTTCTGCTCGCTTGTGGCCATGGG A GGTTA T TAA T TACAGGAGGCTGAAAAAAGG CCTAATTTCTGTTGAGAATTATGCCAGGCGAGTCG T TCAGAACCAAGAATTTGGCATCGTTGATAAG T A C A T C C G A A G C A G C A G T G G G T G G G G A AAATTGGTGTTGACAACCCTTGAAAGGTTTTTCTA CATCAATGTGCCAAGTTTTTGTTCACCTGGTTATCG AATCGTATTTTGCCTTAGGTAATTTGAATCGCAAG CAGTAGCTCTAGCAGGTGTTGGAAGAAGTTTTG AACAGATTGGATTACGGTGCAGTGACTCCTTTG CA T T GT TA TAAAGTTGCATAATGGACTCAAG TCTCAGGTATA T G T A G T GGA T TCACAGGATG CTTCAGGTCACTCATGAAGAACTTGAAGGATTGG TTGCATTTGCAGCCTAAAACATTGGATGCCATTG T GA T C C C A C A T G T C G T C T T T G C T G T A T C T G TTTTTCGTAGTCATCTCTTTTCAAACACTAACAA TATTGGAACTCAGGAAATCGGTCATGACGACAT CAGCACTTATATGGAACAGGAAAACAGTGGGG TCTTGCAGAGGCATCGACCTTGGCTTTCAGTCGCTG AGCTGCTTGAACAAAACCCAGTCAGGATTAGAAGG AAGAAATCCGGAGGAGTTCTATTTGCCTTTTCAA CATTCCACGTAAAGGGTCCTCAATCCACTGTTA CA A A C T A C C GGGGACCACCGTACGCA A G ACAAGGCGCACTCCACGTGTCAGTCATTTATC CAAAGCAGGTGGCGTTTTAATTTATCAGCCTTTGG ATTAGACTGACGGCTGAGTTTTCGTTTTGCTGCG TCGCATCGCGCTAAAGAGGATTGTCGAAACCCTTT C A G A A T C T C A C T T T C A A G T C T G C C G C A G TCСАCTCTCTCTCTCTCTCTCTCT

LEFT PRIMER AGACTCCGTTCAACATCAAA RIGHT PRIMER GCTGTCTCTGTTTAACCTGA PRODUCT SIZE: 184

\section{G1_Unigene_BMK.5644 p5 (GAGAC)4 2011921211}

CGAGTACTCTCACTGAAAAACAGACAATTTTCG ACAAGAAGTTCCGAAATTATAATCATTAGTTAAA CAGCACAAGCTGTATGAACGCAGTCAGTCTGG ACATGATTACTAATAGATATTCGATACTGTCACCATT TAAAAACTACAATACAGGGAAATTAACTTAAAAC TAGAGGTCATGTCAAATACTTATTGCGGCCCAGTGG CT GGCATATAA GCT TCTCCTGCTCCT GAACT T CACGGGGAAAAAACAACCAGACCT TCCT TCAC TAATGAGCTAGGGTAACACTTGATTTTCTTGTCACAG 
AGAATAGTATGATGCCCCTTCTCCATAGCCGATAATG CTGCTTCTCTTGTGCTGAATTCCAGATACCCATAA CATTTATCTTCATGGTTGTGCACCATTACTTCTATAA CATCTCCAATTCCAACATCAGGTAATAAATCACGG AAATGACGATAGAGATCAATTTCAGTAACCTGTGG AGCTAGATTGGCCACAAAAAATGAATAACGAAACCG T T GGAA T T C T G T A T T T GCC T T C T CA T T A T CA T CACCCTGACCATTATTGCCATCGCCAGCATTTTCTAG T TCTATGACAGCTAGAAGATCCT TCACCCGAG AAGGGCAAATGTGAGGATAGTGTCCGTTTTCGTTA CAATATAGACAGTCCTTTAACTGAGCAAATGTATG A T GA TCACGT T T TA T GT T GCAAT ACT GCGACT CAATGGAGCACATGTAAGTATGACAGACGCTACA CATTATTTCAGCACCAGGTTCTACTTTTGTGCCAGG AGGGCACGATCGAGCCAATGGGCACCATGCAATG TGGTGGTCATTTTTACCACAAATGCTGCAAAAG TAACCTTCTTTCACAAGAGGGCGAGCAACCCAAG TCTCTCTGGGCTTCAGATAATTGGGGCACTCCCAAG AGGTGTGCTCACCAACGTAGTCACAATAAACA CAAATCTCGCTGCTGCTGACACTATCATCAGAACTA TAGTAATCAGAATCGAGAACCATTGTATCCTCTG ATTGCTCCTCCCCAGGACAGACTTGATTGACTTCTTG TTTTTTCTCTTTAACACGGAAATCTTGATTAATT TCTCTCTCCCTTTTGCGAATCTCCTTATCCTTGCAC GGGCT T GA C GA GA GA GA G T T GC T T T C CA T GG CACCGGCTTGACAAGAGCAGAGACGAGACGAGACG AGACCAGACGTGTGTAGATGCAGAGGAGATAA TACGGTGCCCAACCTTCAAACAACCGTCATCTG CACTTGTACAGGTAAAAAAGGAGTTGCTGTTA TAAAGAGTTGAAACATGAAATGACCCAG

LEFT PRIMER CGAATCTCCTTATCCTTGCA RIGHT PRIMER CAGATGACGGTTGTTTGAAG PRODUCT SIZE: 152

\section{G1_Unigene_BMK.5855 p5 (GAACA)4 20140159} GTTGAGTATGAGACGACTCCAACAGTAAAAAGTAAG A C A GA GCGG T C A A A A T T G A GA A C C A C T C A G CCAAAATCCGTCATTTGTTTTACAGAAATCAAATT TTGAGTCCCAATTGCCCAACTTTCAACAAAAATAAG ACGAACAGAACAGAACAGAACAGAGTCCCGTTACCA T A C GCA C GC C A A G GC C A C A G A G G G GCACG AAAATGGTGTTCAACAGCCTGATGGTGGAGACGG TGGCGCACCTGTCGGCCGAGATGTGGCAGTACTTGG CATGCTTACCGGAGCGCTTGAGCAGCCACCGGTTG TTGGATCTCATCTTCTGCCTCCCTCTGCGGCAG TTGGGTCGCCTGGCTTTCTGCCTCTGGACCTTCC TCTGCGTCCCTCCGCCCGACTCCTACCACT AC TACTCT TCC TCCGACTCCAATGAC TCGTCCAC CACCGTCGATTACGATGACTATTACTACGATCCC CACTCTGATTGATCCCCTTCTGCTTCTTCGATTG TCTTCTCCTTCGCCGGCTCCCCTCTACAGAAGATG CAGGATGGAAGATGGATTCTTCGATAAACTCTTTCG TTGCAATACATTGTACGGTTTGTCTGGGAGGGGG ACATATAATTGAGGAGCAGGCTTAACTTTGTGG TAGTTAGATTACTGGTTGCTGGTGGATGAAAAGG AGAATTACTTTCTGAATACAATTAAGTTGAGATCC CA T TCCTGTGT T TA T A T GAAAGCT TCAAACAG TCTTAGATCCTTTGAGTTGTTGATATTACGTGGAAG CATCGATATTTGATCATATGCTGGTGATGTTGTATT TACAAAT TGTACGAAAATACTGGATGATATGG TTAATAAATAGTAACACTCAACGTTTTACAGCAA
LEFT PRIMER AGAACCACTCAGCCAAAATC RIGHT PRIMER CTGTTGAACACCATTTTCGT PRODUCT SIZE: 166

\section{G1_Unigene_BMK.6116 c (GGAATT)4ga(GATTGG)3 44} 227270

GAAGTGATGTAGAAAATTGCTGACCCATAAGTACCG CGAACATGTTGAAGTTCGTACCGCAAGTCAATGGG CGCCTTAAGGATGTAGTTTGAGGGCCCGGTGGG TTTATTGGGGCGTTGTTCCTCCCGACGACGATGG TCCGCGTGACTCGGCTCTGGGATGTGAAAGGAAG AAACTTTATTTGTGTTTGGGATTGAAGCAGTTAGGG T TAGGGT T TGGGTCGT TCGGAAT TGGAATTGG AATTGGAATTGAGATTGGGATTGGGATTGGACA CAAATAAAAGGAAGCTTGTTGCGATTCCAAAT CAACTGTCGAGCAAAAATCCCTAAT T T TCTCG TCATTTTAAATAACT

LEFT PRIMER CTGGGATGTGAAAGGAAGAA RIGHT PRIMER TTTGCTCGACAGTTGATTTG PRODUCT SIZE: 166

\section{G1_Unigene_BMK.6539 c (GGT)6agtggttgactgecgecggagca ggaactttggecagcattctactatetgtgtgtgaaattgtgatacaaagtgaaagtgc aagtc(GTTGGA)3 12422562379}

CCCCGGGCCAGCGTTGTACTTCACTTGATGTTGAAG AACTTTTGTGAGAGAAAAATATAAGGTGTTAG TTCTTGTGGCAATTCTAACTCAGGTTTTACAAGCAGG CAAAAACCCGTTAATACAGAAGCTCATACGCAG CTGGTACA TCA T GCACCGGGTCACCTT T TCGG TTTСтTTCСATTATCTTCCGGCAAGGGAGACTTG T T TCCCCACCCCAAAGATGAATGCCTTCCCTAT TCCTCCAGCACCTCTGGCTGTTCTTCCTACACGTCA CACACACTTACTTGTATGGCAAGGGAAGTCGAAG AGTATAACATGATCCACACCCGAAAAATCAATTC CACGCGATGCTCTGACAGATATTTCATAAACGG CAACTTGCTGATTACTGTATGTAAGCAAAGTTCAG TACAGGAAACGATGCAGAAAGCAAAAGAAGTAC T A C C T A T C A G T G C A A A C C A A A A A C T G T T A ACCTCCTTTGAATTAGAGTTGGTGAACTCCTCCA TATTTGCGAGTCTTGATCCTTGGGCCAAGGCAGAG T G G A A T G G T A G A A C C C G A A C G C G A G T T C C ACTTCTGTCAAAACGTGTTAATATATTCTCAACT T T TCTGCACGTCTCAATCT TGT TGCAGAAAAT TATCGATTTGGGAACTGGACTTCCCTCCACAAG CTGTAGAAGAGCAGATTTCTTATTTGAGAATG CCTTCTCTGGAGATCTTTCACT TCCATCATCTC CACTGCAATCTACAAGCACCTCTTCAAGGCCAGAG CTTATACGGTGCATTCCAGGTGCCATGACTACTTTA C A A T C A G G A A A G G A T T C A A C C A A T T T G T T A TAAATTCCAAGGGGTAATGTTGCAGTCACAAAAAG A T A T T G C G T G G T A A C A G G T G C A G A A T T G ATTAAACTTTCCAGTGCTGCTTCAAAATCCTCAT CATTAAAGAGTATATCTACCTCATCCAGTACAGCG CATCTTAGATTTGATAAGTGCACGAAGCCCTCTT TAATCAGATACATAAACCGACCAGGTGTTGCTAT TAGGATATCCACACCTTGTTCTAAACTTTCCATCTG AGTTTTCTGCCGATGACCACCAGTTACAACCATAG ACCTTACTGGAACCCCAAATTTTGACATTGAACGG CAAACACCCAAAACCTGAGAAGCTAACTCAGCAG TTGGTACCAGTATAACAACTCGAGGACTCATTG A C GA G G A C T T G C T C A GC C C T T G A A G T T C T T 
CT TCCCTTAAACGCTGAATTACTGGAGCAAG ATAAGCTAATGTCTTGCCAGATCCACTTTGGTCTG CTATAATAGACGTT T TCGCCTCAACAACAGG ACCAAATGCCATGGCCTGAATATGCGATGGGCG CTGGAAAAGCTGCCTCTTCAGACATTCAATCATA TATTCACTGCAACCCACATCCCTAAAGGATTTTCG ACTGAAAA A G T CATTGTCGGTGGAAAATT TC CTTCGCTGATTTAGGTCGGGCAACTCAGCCAAG T C A T A C A C T G A C C C T C C T C C A T T G C C C C A TCCTTTTGAGTGAGAAGAAGAACCTCTTGGAGCTG AATCCTTTTGTCTGGCTATGTTGGTGTCAGACCTA TAAGGCTTATGTTGTTTGTTTTGATTGTCAGAG ATTGGCTCAAAACCATTTTCCGTCCTTTTCACAGG A G A A T G G T C C A T G T T G T T T G C T G T C C C CTTCCTCCGCCTCATGTCCCTAAATTCTTTCGAG TGGGAATCCCTTGAGCGTCTTCCACCAAGACCG CGGGCATCTGCTCTGTTCTTTTTCCCATCAAATT CAAGTCTTGAAGGCGAATTGACAAATGGTGGTG CATCTTGCACATTTTCTTCATCATCACTTTCATC TATTTCTTGCTTAATCCGAGAAGGTTTCTCCGCTA TAGATTTCACTCTATGTACCTTCAACCTCCCG AAACTTTGAGCAACTCTCCTAGTAGTAGGTG CCGGTTGACGGCCACTGCCTGTAAATGGTGG CACATGCTCAATTCCCACCCAAACTCGATGGG TGGTAGTGCTTCTGGTGGTGGGAGTGGGAGGGG CATTGTTGTCTTGTCCGAGGACGATGAAATCG TCCATGAGGTCATAAGAAGCTTCTGCTGCAGCTG CAGGGTTATCGGTGGGTCTCTGACTGAAACTG CAACTAATCCCCCATTTTCTCGTCTGATGAAG ACAGAGACACTTGCAGCTGTGGGTTCCCGCCATC CATGATTCTGGTTCAAGGCGTTTTGGCCGCAGG AGGTGGTGGTGGTGGTGGTAGTGGTTGACTGCCG CCGGAGCAGGAACTTTGGCCAGCATT TCTAC TATCTGTGTGTGAAATTGTGATACAAAGTGAAAG TGCAAGTCGTTGGAGTTGGAGTTGGATGAGGTG ACAAGTAACAACGGTTATCTCTTTGGTGCTGG TCTGCTCCTG

LEFT PRIMER CCATCCATGATTCTGGTTCA RIGHT PRIMER GACCAGCACCAAAGAGATAA PRODUCT SIZE: 204

\section{G1_Unigene BMK.7028 p5 (TGATT) 4205877} GGGCAATGTATGCGGCAAGACTTTATTTATGATGTG AGATTAGGTAAGATAGGCAGCTGATTTGATTTG ATTTGATTTGAAGACTTAAAGAACAAAACAAGTAA CAAATTTGGACAATCAAAGAGGGAGAATACAATG ATCACCACCCCAGTCCATCAAAATTATAAACAACA CAAAGAAACAAAGCAGCCCTCTAGGCCTTGGGTG CTAGCTTAGACTTGATTTTGTCAACCTGCTTGG TTCCCACCTGGAATGTCTTGGTCAGCACATTATCCGG CACCTCCGGCGTGGCAGCGAACAACGTGAGGGCG ATGTTCTGGGTGCCCTGCAGCTGAGAGTTAAATG CGGCAATCACCGAAGCCGGACTCTTCCCATTGTTG TTCTGGAAGTGAACCAACCCTTTGGGGAACGCAAAG ACTTCACCTTGCTTAATAGTTTTGCCGATGAACTTG TTTGCTGTGGTAATAAATCCAACCTCCAATACTCCTT CAAGAACGTAGACAATTTCTGTGGCTCTGGGGTG AGTGTGCGGTGGGTTAATTCCGCCGGGGGCATAG TCTATTCTGGCAAGCGAAACGCCGAGGGTGTTG AGGCCGGGGATTTTTTCGACATTGGCTAAAGTCAC CAGGGAGCCGAATGTGTTGTTGGTGAGGCCTGG
TTTGGCTAGTCCGGCGAAGAAAAAGTCCTCTGCTG TTGCATTTGCTGCGTCCTTGCAGACGAATCCATTG A C T T T C A C C GC GGCGGT GA GG T C G GC GA C G CAAATGTCCTGAAGCATGTCGGGATCAGCGGCAG AGCTGCCGAAGACGGTGGCAAAAGCCACAAG AAAGAGAGCAAAGACGGCCGCCATTGGTGGTGG TGGTGCTAATTAGAAAGAAAACGCAGGGTAGTG CCAGAAAGGAAGAGAAAAGTCTGGATGTGTGAATG ACTAGGAAATGATTGGTAGGATGAGTATTTATAG CAAAGGAGTAGGTGAATGTTGAAGCCTTTGGTACG TATTGTGGTAAAT

\section{LEFT PRIMER AATGTATGCGGCAAGACTT} RIGHT PRIMER TAATTTTGATGGACTGGGGT PRODUCT SIZE: 160

\section{G1_Unigene_BMK.7859 p6 (TCAAGA)4 2416511674}

AAAAGAAGCAAAGCAAAGAAAGGCTGCT TCG CTCTCCATACTAAGGAAAGGAGAGCGGTTTTATTAG AACGTTAGGATGGAGCAGGAGCTCTTCTTTTCGTGG CTTAAATTTGTTTCCATATCTTTAATGGTGTTGG TTTTTTCTATGAGGATGGCGGTGGTTCTCTGGTGGAGG CCCAGAAAAATTGAACAACATTTTGGGAAGCAAGGG ATCAGAGGACCCCCTTACCGTTTCTTCATTGGAAATTA C A A A G A A C T T GC T G GC A T G A T G A T C A A G G

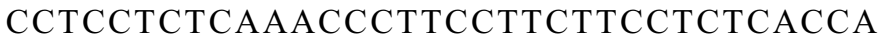
TATTCTCCCTAGAGTTCTTCCTTTTTACCATCACTGGAG AAAAATCTATGGTGCAACATTTCTTGTGTGGTTTGG ACCTACAGTTCGACTCACAGTTGCTGATCCTGATC TAATTAGGGAAATATTTACCTCGAAGTCAGAATTT TATGAGAAAAACGAGGCTCACCCACTTATTAAACAG CTGGAAGGTAATGGCCTCCTAAGTCTCAAAGGTG AAAAATGGGCTCGCCATAGAAAAATTATTACCCC TACCTTCCATATGGAGAATGTCAAGTTGTTGATACCGG T G A T G G C A A C A A G T A T A G T A G A C A T G A T G G ACAAATGGACAGCAATGTCCAGCTCCGGTGAGGTAG AGATTGAAGTTTCCGAGTGGTTCCAAAACCTAACAG AAGACATAATTACCCGGAAGGCGTTCGGAAGCAG CTACGAAGATGGAAAGGCCGTTTTCCATCTACAAG CTCAGCAAATGGCGTATGTCACCGATGCTTTTC AAAAAGTTTGCTTCCCTGGTTACAGATTCCTGCCCA CAAGAAGGAACATAAATTCTTGGAAATTGGACAAG CAAATTAGGAAGTCATTGATAAAGCTGATCGATCG ACGGGTAGAGAATTCGAACTCGGGCGACAATGTG CATGAAAAATGTCCAAAAGATTTGCTAGGTCTTATG ATTCAGGCCTCAAATTCTTCCCCTTGTTCCAATAT CACTGTCAATGACATTGTTGAAGAATGCAAGAG CTTCTTCTTTGCGGGAAAACATACCACATCCAATTTG CTGACATGGACAACGGTTCTCCTAGCAATGCACCCA CAGTGGCAGCTACAGGCACGTGACGAGGTGCTGAGGG TGTGCCGATCACGTGACATACCCACAAAAGATGATG TTGTAAAGCTTAAGACGCTGAATATGATCCTCAATG AATCACTACGATTGTACCCACCTGTGATCGCGACG ATCCGGCGGTCGAGAACAGATGTGGAGCTCGGGGG ATACAAGGTCCCACGTGGGACCGAGCTTCTGATTCCG ATCATAGCCGTCCATCATGATCAGAGCATATGGGG CAATGATGCGAAGGAGTTCAACCCGGGACGGTTCG CGGAGGGTGTGGCACGGGCCGCCAAGCACCCGGCGG CGTTCATTCCATTCGGGCTTGGGGTCCGCACATG CATCGGGCAGAATCTAGCGCTGTTCCAGGCAAAAC TAGCCCTTGCCATCATGCTTCAACGCTTCTCTTTCAGG CTGGCCCCCACCTATCAACATGCACCTACGGTACTG 
ATGCTACT TCACCCACAATATGGGGTACCCAT CATTTTCCAACGCCTGCCACAGCATGATCAGGG TCAAGA TCAAGA TCAAGATCAAGATCGAAAT CAAGGGCCATGATCTCGTATTGTCACTATGCAACAC CAGTCATTTGCGTGTGAGTGTGCCGTATGATTGG A A T T G A A T T G A C C G A A A T A C C C T T T C G G CCTTTTTTCGTTTTGTTTTGTTTTCTTTTTCTTTTTGTG CTGGGTTTCCTTATAACCATAGAAGCTAAGCTTAT TAAGGTCAAAGGTAATATCTTAAAAGAAAGATGTTG CACTCCCTTTCATCTAGCTTTTTACTGACCCTCATG A C C A T G G T T T T C T T A C C A A A G G G G A A A A G AAAAAAAACTAACCCTG

\section{LEFT PRIMER GTACTGATGCTACTTCACCC} RIGHT PRIMER TCAATTCCAATCATACGGCA PRODUCT SIZE: 178

\section{G1_Unigene_BMK.7950 p5 (TTCAA)4 20496515} GTCGAAACACGGTTCACTACATTTAATACAATG CATGGTATCACTCAAACTTCCATGTGTTCCAACAA CAAGGTAATATAATTTCTCTGACAGGGAGATGGG ATTCCTATTAACTAAGAGCTTCAGTCGACAAAGTG CTGGAGTAAAGCTTGTAAGCCATGATATGATTCCAA CATTTGGTCTCCAAATTATTCAAGTAGGCTTCTCA TATGAATTCTGGTCTCTAAATTCTGCAATGGCG C T T C T T A T C A T C T C G G C A G C A T G G G G C T G CACAAAAAGGTGAGTGCTATCCAAGACATGTAT TATGAACCTCTGTGAAGCTGGCAGTGAATTGTCG TAATTGATGATGAATTGCGCCATAGGTATGTCA CAAGAGATGAACAGTCCTT TAACAGCATTCAC CATCTTGTATCAGAAACCTCAAGATCTTTTGTTGCAG AGGTGGTCGGAGATTTTGTCCGTCTCCGTCTGCAT CAAACGGAGGCCCCTGATTATTTCAATTCAATT CAATTCAATTCCTATTTGCCTTCTTGTAAGTGGAGG CCCCTGATTAATTCAATCCAAACCTGCATCAAAATCC CAAATCAAAGAACGAGATAATATCCCAGATTGTG TAAAAATTAAACAGGAAATGGAATGCGAAAGG ACATTTTCCTACAGTTTCACGGTCTGAGATGGGGGTG TTTAGATTCGATGTGCGCACGACGGCAGCTCCACAG TCTGAGACGTGCTAACAGAGGCGCACGGTGCGAAG TCCGCGCGGCTGGCTGGGTCTGTGGCTGGGTTTGTG ATGAAAAGACCAAAGAGCCTTTGAAACGCTTG C A A A A T T A A T A C C C C T T T C A A C C T A G T T C A CAAAATCCATTTGTAAGGGAACATGAATCACCTTTA CATGAAATAAATTTTAGAAAAATCAAAATGTGGG AATAAAGTGAA

LEFT PRIMER GATCTTTTGTTGCAGAGGTG RIGHT PRIMER CTTTCGCATTCCATTTCCTG PRODUCT SIZE: 221

\section{G1_Unigene_BMK.7991 p3 (ACC)7 21243263}

TATATGGTTTCAGAACAGGAGGGCGAGGTGGAAG ACAAAGCAGTTGGAGAAAGACTATGATGTTTTGAAG AAAAAATTTGATGCTCTTAAGGCTGACAATGATG CTCTTCAGGCTCAAAATAAGAAGCTTCAAGCTGAG TTATTGGCTCTAAAAAGCAAAGATTCATCAAATG A A GTGGGGT T T A A C T T C A A GA A G A A A T G ATCAGGGTTCTTGGAGCAACGGAAGTGAGAACAG TTCAGACCACCACCACCACCACCACCATCTCAACC TAGAAATTTCAAGAACACCGGCAACCACCAGCAG CCCGCCTTCTGATTCTCCTCACGAGATGAATGG AAAAAACCTCTTCCCACCGTCCTCCTCCTCCCTCAGG
TCTGCAAGCACAACCCAACTCCTCCAAGGATCAT CACGGTCAGATCTTCAGTGCCTTAAAGTTGATCAG A T G A T T C A G G A T G A A G GC T T G T G C A G C A T G TTCAATGGGATTGATCAAGAGCATCAAGGGTTTTGG CCATGGCCTGAGCAGCACCATTTTCATTAATAAATTG ATCTTCCAATTATTTCCAAACCCTGAAGATTAATTA CATTATTCTGAAGCAAGTTTGGCCAAGGCTCTGTG A TCAGACAAGTAATGAATTAAAAATTAGGAAA TTTTTTT

\section{LEFT PRIMER AGTGGGGTTTAACTTCAAGA RIGHT PRIMER GTGAGGAGAATCAGAAGGC PRODUCT SIZE: 154}

46. G1_Unigene_BMK.8037 p3 (CTG)7 21708728

GAGTTTGCACGCTCGGTAGGATTCCACGGGCCAG CCGAGGTATAAGGAGTACCAGAGTAGGTAGAGG AAGGTCTCGATGACTGGGGAGTAAAGTCGGCGAAG CTGCCCTTGTACTGTGGCTGGAAAGGAGAACGCG A GGCGCGTGGTGACTGGCGCTGGACGTTCGTG CTCAGTGGGGACAAGCGAGTGGTGCGCGCACGGCTG ACGGCCTGGGCATCATATTCATTCCAAGCACGCAAC CAGGTAGCCAGACCAGCAGGGTCAGTAGGATCACAG TGCAAATCGAAGGAGATGAGGGCAAGCCATTC CATCTCGAGGTTATTCAGCTCAGACACCTTGATG CCAGTGACGTCGGACCAGGAGCGGTTGATGAAGGTG TTGTCGTCGAGGAACTTACTGCCCAGAATCAGGGCG ACGGCGAGCAGGCGGTAGATGTGATTCTCGGACG AAGAGGTAGTCCTGGGGGGATAGTCTCGCAGACG AACGGTCAAGTAGTGCAGACTGAGGAGGATGGTTG CCGAGGGCAGTCTGGTGGCCGACAGGACTTGCGAG ACCCACTTGCGGAACGAGGGCTGGATTGGAACAC TAGGCTGGATGATTCCCGACGCCGATTCGGCCA CAAAGTTGGTCATGCGTTCCATGTCGTAGTCGAG CTTGGCCGATACTCCACCTGTTGTTTTGTCT TC CTTCTGTGTCGATTGCTGTGCCTGCTGCTGCTGCTG CTGCTGCCTGACCT TGCCCATCTGGGTCATCTG AATCCCCTTGGCAAAGTTGCGG

LEFT PRIMER AACACTAGGCTGGATGATTC RIGHT PRIMER GGATTCAGATGACCCAGATG PRODUCT SIZE: 179

\section{G1_Unigene_BMK.8106 c}

(CCACCT)3cetctttetcetegtacace(TCCTCG)3 56392447

CGCAAGGCAATCATTCATGAAACCATTTCGTTA CAAGAAAAGTACAACTTGAAACTTCAACTCAAATA TACCAAATACCAAAAACAAAAAAAACAAATAAT CAAATAACAAAGATTTCTACTAATAACTCCAAT TAAAAGAAAATGCATAAATCGAATTTGCTTGCAG TAACTTTTCTTTTACTTTTTACTTTTACTTTAACTT TATCTGGTTCAGAAAATATCCAGAATTTCCAAGATG AAATTGTTGCAGAGGGGACAATTGCCCCGCTGG ACCCACAGCTCTCGGGAGCACATCCTGCAGAACGTG TGCCCGCAGGGAATAAACGCCGCCCCTTTATG CCTCACCATGCACACGCAGCAGTTATACTCCA C A T T C C C T T C T T C C G C C G C C A C C T C C A C C T C CACCTCCTCTTTCTCCTCGTACACCTCCTCGTCCTCG TCCTCGTCCATGGT GTACGTCGACTCCACCCC CATCTGCCTGTCCGTCTCCTCCAACAAGTCCAT CAACGACATCCTCACCGGCTCCGCCGTCCCCGACG CCGCACCTGTCTCTCCCGACGACACGTCATTTCCCG TCTCTTCT TCCGCAGACAATGCCGCCGCGGAAG 
CTTCGGCAGCCTCTTGCGCCGCCACGGCTTCCCTGG CCGACAGCGATCTCTCATCGTCCAGCGCCGCCG ACAATCGCCGTCTTGGAGAGTCCGCCGCGACCTCG TCGGCCGCGAACCGCACGGAGCCCTGGCGCGAG AACATCGAGGCGCGGGGGTTGTTTATATCCACCTCG TCGGGCGGAATCGAGGGGTTGGCGCTGAAGCGGG TGGAGCCCCGCCGCGAAATTTGCCTCTCGACCTGCTG AGTCATGTCGAGATCGGACTCGGGAGTGACGTAG CGGACCGAGCTGCGACGCGAGTTGAGAGACCGCGGG T GGTGGT GGT GA T T GGAACCGTAG T T A GCA G TATTCCGGATGGGGATATCGGAGGTGGGGACACGG ACGGAGGAGGTCCAGGCGGAGCCGGCGCGTTTGAG TAGGAGGCGGTCTTTGAACGACTTCCAGGCTTTC T T A T C C T T C T T GC CA A T A GGGCC C GGT A GG ACTTGGGCTCCTCGTCGCGGATTATATCGAGCAGGG TCCGACCCGAGATTGTGGCGGGAAGTGCGGGCGG TGGAGGGGGCTGTTTGGCGGCTGCAGGGGCCAGCG A C A C G T C G T G T A G G C T C A A T C C G G C A A A G TCTCTCCCGTTGCTACTTGCTGACATCTGATCGAG A A GCG T T A GC C T C C G GC G A C C T T C C A T T T C A TAAAATCCACACACAAAAAAAT

LEFT PRIMER GCAGCAGTTATACTCCACAT RIGHT PRIMER AAGAAGAGACGGGAAATGAC PRODUCT SIZE: 230

\section{G1_Unigene_BMK.8119 p3 (AAT)6 1897114} GGGTCACTTTGGGTCAACCCGTTAGCACCCGTTAG TTGATGATGTTTTAGTAATTTCAATAAAGTCTTAA CAACAAAAAATAAACTCTACGCAAAAAATAATAA TAATAATAATTGTTAACGGATGAAACGGGTCGGG TTCGGGTGATCTATTAGCTTAACAAGTTGGGTTCGGG TGACCCGTTAACTTAACGGGTCGGGTTCAACCCG AACCAAACCCAATAAACCCGACCCGTCAGCTCCA TAA T CA T GACACAAGAGTATCAATCGTCTAA CATTTCCCAACATCATTTAGATATAGGATATATAT TATTATCAAATCTAAAAACTGGGAAACACACAAAA CACCTAATTCACAATGCCACCTAATTGCATTATCCG ACT A A A T T A GT A A C C CAGTCTCAACAATAG ATCATGCATATGATAATTTCAATTTTATCAGACTGGG C T A A A A A T C A T T GCA G T C T C T T G G A C T G T G ATTTCTCGTGGCCACTACATATTAGAACAATAGGTG TAGGTGCTACTTAGCCTTAAATTAGCAGAAGCAG CTGAGCTCGCAGCTAACCAAGTAAACATGGATCAG TAAACCAGCCAAGTCACGCTCATTAAGGTGGGTG CATTAGGCATCTTCCAACAAAACAAGTCATATTG TAACGCGGTTAAGGACAGACAGAGAGCTTGTTTCA T A A T A T T T T C A A T G A A G G A C T T G A A A T G A G TACTTCTTTGGGTGAGTTGAGTAACACAGAAGAGTG TAGTAGTGGCACCGACGACATACGAAGTGTGGAAAG T C T T G G A G A G A C G G G T A T A G C A T G G C T C A CAAACCTTTTCAATAAGATTTTGAAAACCAAAAAG ATGCCAAATGAGTGGTGGAAAAGCACATTGGTG CCAATCTACAAGAATAAGGGAGACATAAAATGTAT TAACTATAAGGGTATTAAGTTAATGAGACATA CAATGAAGCTTTGGGAGAGAGATTGAGTATAG A A A A T CA T T C GGGT T CA T GCCA GGT A GT T G ACTAAGGAGACATACAAAATTGCATCAACACGTG CAAAAAAAAGTGTAGCAGAGATGGGAATGCTTTG TTGGATGTGTGGCCACATGAGAAATGACAGGATTG AGAACTAGGATATTCGAAGTAAAGTAGGAGTGGCCA CAATTAAAGATAAGATGAGAGAAAAGAAATTAAGG
TGGTTTGGACATGTAAGCACAAGACCTATAGATG CTCTGGTTTGAAAATTTGACTATGAGACAAAGG CTGGGGTAAAAGGGGTAAAGGAAAACCTAAG AAAACTTGAAAAGAGACTCTAAAAGAGACATGGAG TACTTAAAGCTAATGAAAGAATGGATGCAAAAC TAGGCAGAATGGCATACTAAGATTCTTACAGCCG ACCCCACTTAGTGGAATAAGGCTTTGTT

LEFT PRIMER ACCCGTTAGTTGATGATGTT RIGHT PRIMER GTTTATTGGGTTTGGTTCGG PRODUCT SIZE: 200

\section{G1_Unigene_BMK.8296 p4 (CAAA)520 132151}

TTTCGATGTTTATTTACGCCCCTTGATTCTTCTTCG ACTTATTCAAAAACCGAAGGGTGTGCGAAAATTG CTTTCATAAAATTTGATTTTTATTCTAAGAAAAG CAATTGCGATGGCTCATTAGCCTAAACCAAACAAA CAAACAAACAAAAATGGAAGGCTTCAATTGATTTA CAGCATGCTAACTTCACACGCTGAGTGTCTAATTTCA TAGCAAGTCAGTGTTGTAGGCCTGAACCTACTTTCCG TCATTCCAAAGTACTCGCTTGTTCTGCAGCCAGTG TAGGCAAGATGGCCATAGCCTCACCGTCAATGGCAG TAGCACCATTCTTGAAGCATGCCGTCTTGAATTTCAC TAGATATCTGTTCTTCTGTTCTCTTATGTTAGTTG CTTGTACCTCACCAACTATCTCTTCTCCAATATAG ACAGGCAACCTGAAATGCAAGCTTTGGGAAACATA TATAGCCCCAGGAAAATGAGAAGATATGATCT TGGGGAACAGGGCAGCTACAAGCATCCCGTGAAC CAGTCGA TCT TCAAA TCCAGCAT T TCGAGCAG CCTCGGAATCTAAATGCAGAAGGTTAGAGTCATG ACTCACTTTTGAGTACTCCAAAACATCTTCATTGGTG AATACTCTATTTTGCTTCAATGTATCTCCACTTC TAAGAAAACCAGCTGCTGCTGACGAAAAACCTCT CAAAGAAGGTAGGTTGACCGAGAGTAAGTTCCGG CCAAGCATAACTGTAACCGTTTCTAAACGCTGTCCAG CAGAGTAGCAGCCCGCCAAAACTACGTTGATTT

\section{LEFT PRIMER ATTTACGCCCCTTGATTCTT} RIGHT PRIMER GGAATGACGGAAAGTAGGTT PRODUCT SIZE: 244

\section{G1_Unigene_BMK.8884 p2 (AG)11 22739760}

CACC CATCATAATAATTTTACTTGATAACATGAG AACACTCAAAAAGTTTACCGAAAATGATCATCAT CAAATTAATTACGTACAACCCTAAACATCAATTAAA CAACACAAACCAACT TATATACAAACAAAACT CAAAGCACAACGTGGCATAGATCGATCATAGTCCG CATACAACAATAATTTACTAACAGTAGTCTAATC TAATTAAGCCAGTGAATTTCAACCCTTCTTAATTACT TATAAATCACTTCACATGATAGAACAAGCATGTGG ATTTTCGTCGCTAAACATAGAGGTATACTTGTCG TCCGGCGCATTCGGGCCCGGAAGTGCCTGAGTCACG TTCCTCACGTAGCCGGAGGGAGCCTTCTTGTCATAAG CTCCGGCGACATAAGGATATGCCACCAAGTTG TAAGGCACGTACGGCCAAATCTCTGCCTTCTTCCCTG TGCTCTTCACTTTCTTTAACACCTTGTTTGGCTCCACG TA TCCCGTCACCGTCACCTTGCTCTGCTT TCTG TTCACCTCCACTTGCTTAGCCCCTTTCATGTTAGAAA CAGCATTTTTGACTCTCCTTTCACAGCCATCACAG TCCATTTTCACCTTGATTTCAACCGTCTGCATTGG CTTCCGTTTCTTTCTTGGTTTTGAGATTGTGAAATAG TCTGAAAGAGCATCCAGAGCACCCATTTCTCTC 
CACTCTGATAATTCTGATCTGAGTTTTTTTTTTTG TTAGAGAGAGAGAGAGAGAGAGAGGGGGAGAGAG CATTGTTGAAGGAAAGAAGAGGGATGAGGAAGTTCG A TCCATGGCCGA T T T TCT TCAAGCGAGAATGG AACCGGAACTGGCCGTTCCTCGTCGGGTTCGCCAT CACCGGAACCCTGATCACCAAGTTCTCTCTCGG CCTCACCGAGGAAGATGCCAAGAACTCGCCCTTCG TTCAGAGGCACAAGAGGTGATTTATGTTCGGTGTAC TAGCCACCTTTGTGCTAGCATTGTGGAGAAGACGG CCCTCGTGCTATTCTCAAGGACCATAATAACGAAAG AGAGATGTTCTAGATACTCTTTTTTGAATTTTCTG TTATGTTTTGGATGACTTGAATAACATGAGAAGAAA TACTTGTATTCATTGGTTTTGAATTTTGTGCGTTTGG CCAGACATTCTTCAAAACATTAAACAAAGTTGTG TTAGAGTTTCACGTTTTTGTGAAAGTGCTTT

LEFT PRIMER ATTGGCTTCCGTTTCTTTCT RIGHT PRIMER TCCTTCAACAATGCTCTCTC PRODUCT SIZE: 157

\section{G1_Unigene_BMK.8968 p6(ATTTCG)4 24163186} GGAAAATCCGGTTCTCTCTGTCTCTGAGATTAATCAG AAGAAACAAACAAACAAACCTCCGCCTCTCAAAACT CACCACTTGGATTTTAATTATTATAACTCTATCCAAT CCAAACCGACTTCTTTATCTGATTGCCTCGATTCTAG CAAAAATTGGCCCAAATTTCGATTTCGATTTCG ATTTCGCAGGATTTAGGTTTTTTTAAGAGTTGTTG ACCTCAATTTTCTAGTTTTCCCGAGAAAATAAGAAAG AAAGATGATGCGGAGGCAGCAGGAACAAGACCAG CAATCTAGGGTTTTCTACGAGCTATCGGCTCTGG TTCTCAACCTCCTCCGATCTCCTCCCACGCCGATT CAATTGTCGGATAACTCCACGGTGATGCCGCCGTCG TCGTCTTCGTCGTCGTTGAGGCGGCCCCCGGCGGCG TCGACGGCGCAGATCTCGCCGGCTGGGTTCGCGTCG TTGATGCTGGGGATTTCGATGGCTCTGATGCTGTG CGGATCGGTGACTTTCTTCATTGGGTTCTTATTGATG CCGTGGGTGCTGGGATTGGTGATGGTGTTGTATG TGGGGGGGATCCTGTCGATCCTCTCCGTCTTGGGG AAGTCGATTCTTTGCTACGCCTCTGCTCCGTCGACG CCGCGGAAGGACATTCCTGCGTGGAAGCTATTGTG AAGGAGAGGGAGGAGGTGGTTCAAGAGAGTTG ATATGTCTTGCAGAAGGACGGAGCAGCAGCAGCAG CACAGATGTTGTTCTTGTGAGAGTGATGCTGCATA TAGGTAAAGGAATGTTGTATAGGCAGCTGGGGCGG AAAATTAAATACGAGAGCTTGTTGAAGTAATTGATG CATGAACAGTGGATAAATGTTTTATTAATACTC TACTGAATATGAGGAAATGGCTTCTGAAATAG TATATTGTTGAAGGATGATGAAATTCCTAGTTTTG AGGAGATGGCGGGGGATGTCACTTAGCCTCTTCGTG TTCTGATATCTGTGTTGTTCAGTACATATACATCTC TATATATCTCTTGTTCTCAAATCTGTAAAAGTTTA T A GC T C A GC T C A A C A T T T C T T CA T T T T C T GG AACAAATGATCTTTCACTTTTTGTTTTGTGCAT ATTTGTATGTTGGGACTGGTTTCTCAACGATAAGG TTGGTAGTAACTTGTGTGGGCAGGGAAGGGAAGGG ATGAGAAAATTGTAGGGGAATTTAAAGTCGTTCACT TAAATGTACGCAATCCATCCGGTGGGAAATGAAG AGGCACGGGATGTG

LEFT PRIMER TCTATCCAATCCAAACCGAC RIGHT PRIMER CCTAGATTGCTGGTCTTGTT PRODUCT SIZE: 195

\section{G1_Unigene_BMK.8995 p2 (CT)10 2091110}

CTTGGATCTCCACTAAAATGATTCCCAAACT TA TAAAGACGCTGAAAATTTGGACTCTCTCTTTCTCT CTCTACTCT GC T T T CGCAGTCCCTCTCTCTCTC TCTCTCTCTTTCСTCCGTTGCCGGCGTTTCAGCAAG TGAAGTCGAACTCAGTGAGTGATGGGTGGCGAG TTACGTTACGAGATCGCACAAAATGCCTACGTAAAG CTAGTGCTACACGCTCTCAAGCACAAGACCTCCG CCGTCAACGGCATCCTCCTCGGTCGCGTCTCCCCCCA C A A C G A C G T C T T G G A G A T C A C C G A T T C C G TCCCTCTCT TCCACTCCCACA TCGGCCTCCTCC CCCAGCTCGAGATCTCCCTCATCCTGATAGAGGAG CACTTTGCTGCTAAGGGAGTGAGCATTGTGGGG TATTTCCATGCTAACGAAAGGTTTGACGATCACGAG CTCGGCGGCATTGCCAAGAACGTCGGCGATCACATT TATCGCTACCTTCCTCAAGCCGCAATTCTTTTGCTTG ATAACAGAAAGCTTGAAGCTTTATCGAAGACTAAGG ACCGCAGCCCCGTAGTGCAGCTCTACACGAAGGATG CATCCAGGACTTGGAAGTTAGTTGGATCAGATGG AAACCAGTTGACCATCAAGGAGCCATCAGCAAATG TTGTTTTATTGGATTACATCTCCACTGAAAAATGG CAGGATGTTGTAGATTTCGATGATCACCTCGATG ACATTAGCAAGGACTGGCTGAACCCAGAACTATT CAAGTAAACCGGCCATCCCTTTCGGTATTGCAAATG CCTCCATGCTTAACTCAGTTTCTCGTGCTGGGTATAG AGGACAAGGGGGCATACATTGACGTCAAGCTCAT CACT T TC T T T T T T G TAAGGAA T T T TCGAGA T G TAAAAAACTGGATATCAATTATCTTCTCATACTTT TACGTTCGACGCCATAGGGTTTCAGCATCTTCTTTTG TGGACCACTTCAAATGAGGTTTATGGTTTGGTTGTAG A A T T GGA A C A A A A T G T C A A T T C A G T T T T GG ACCTCAAGTTATCTTTCCTTTGCATCATGTTTTCTTG T T GA C T G G G T T T A C A A T G T T A T G T T G T T A G AATACTGGGTTGAAGAGTTTGTTGTGTACGTATTG TTTTCGTATAATATCCATTACCTCAATGTGTTGTGTTG T GGCAA A C T C G T T A T C T T A A T A GG T GA T A T G ATACTGGCCTGATTTGTTAGCGGGTCGTGTCATTTCG TTTTGTGTTGGGTTAACGGTTCATGCAAG

LEFT PRIMER CGCTGAAAATTTGGACTCTC RIGHT PRIMER CACTAGCTTTACGTAGGCAT PRODUCT SIZE: 173

\section{G1_Unigene_BMK.9351 c (CTCTTT)3gag(ACCTAC)3 39} 435473

GAAACTCAGTTTACTTATGGTGCAGTTTACATTCAAA TAACATTACAATTCAACGTTTA

AGTTGGTATTTGCCAACAGTGGCGCCCTTGATATAA CAAATCACTTGTTTAAAAGAGCCTCATGCCTACT TAAAGTGTTACAAGCATTTTAAACCGATAAGAG TTAAAAACACTAGCAAGGAGAATTGTATCACCAA CATCCACTTTGATAGCATAATGTAGTGTATGCCGCAG CATATGATCCAATCGCAATGCTTGATTTCTCCGG CAGGTTTATTGAAGTTTACTTTGCTGTATGAGTGG CTGTTGGGGCTCAATTTCTCCGGCAGTCACAGCCG CAGCCGCACCTGTAGAAGTAGTGTCATGACGTTGG AGAGAAGATCCCTCCTTGT T TCCAACT T T TAG TTTTTGAGCCAATTCAAGTGTCACTGAAGCCTCTT TCTCTTTCTCTTTGAGACCTACACCTACACCTACTTG AGTAGAACCAGCACCAGCACCAGAACCAGAAC CATTCGCATTAGCTGCGTTTCTCAACGCCTTATT C T T T T T G GC C G A C C G A G C C C A C C C A A C A A G 
CCCCTCTTGTATATGTTCTTCAAATATAGCCTTCTTG AACGAACTTCCCATCTGTGTGACAATTGCGTACAG TGGCAAGGTACTGTAACTGCAGACGAACTGAACG AATACTCCAATTACAAGCCTTGGGATAACATAAC CAAGTTCCCCCATCATGCAGGAATGAAATTTATACTG AACCAATATCCAGAAGAAAAATGCGAGTTCAAACG AATTTTGGAACAAGATGATATGAACAAGTAAGAG A A C GA G T C T GGGA C GG T G G A A C C A A A A T G ATCATCTGAAGGCTGAACAACCAAGTCACCTTCG ATTGCAATGTGTTTCTCAGCAACCTCATGGGCCAG TTGACTTATTACATGCTCCAATTTAGTCCCAACAG CAAGTAAAAGTATGAATGGAATGAATGCTATCCAG AAATAGGCATGCCAACCCGACACATTCAGCAACAAG AAGATGACCACAAAGATCCAAAGATACCAACT TATCCCAACGACTCTTTTAAAGTCTGCTTCAAGGG CACGAATCATGTACTTGTGGAAATTAAAATTTGG ATTTGTCTTGCAATGGGTGCTGATGAAGCCTAGTCG CATTGTCATATAATCTGTTTTGGTCACAGAGCCA TAAAATTGCTTGAAAAATGCATGCAACCAGCCAAG AATAACTGAATTCTTTCCAATACCACGAAAGCGG CCCCTAATAAAATCATGATCGTGGACGTGGGTG A A C T T C A T T G T C A A A A C T T C T T G T G G G T T G TACTCCT T T T TGGTGATAGAATCCTCCCAATG TTTCCATTGACGTATCTTTGCTCCTCCAAAAAG AATGGTTAGAACACAGAAAGTCACATGCACCACAG CCAGCACAAAAATGAAGATGTGAAGATGATG CAA T GCCGTGGTGGATAACA GA GGGACC T T C CCCTCTGAACAGGAGCCGGACGTGGTGGAAGCAG AGTCTTCAGCAAGAAGGCGACGGGCAGCACTG CTCCAGGGAAAGAACACGGTCTGGAAATGGGCAC TAGTACTAGTACTGGTGGTCTCTTTCTTGCAAGG CAGCCATTGACCGGCATGTTTTTCGGTTATACAG ATTTTGGCTATACGTCCTTGGAACACCGTCAGCAG CAGTGATATAAACCCCAAAAGCATCAACTCTTC T T T GA T C T T C T G C A A G G C C T GA A A G A G A G G TTTCTGGTTGTGTTTCTTGAGATACTTGCCAGTGTAG TGAAGGGTTCTCTCGAGGAAAAGTGAAAGGATG ACTATGACACTAGACACCAAAGCCACCACCCAAG TCGGAGTGTACTCCAAGGTTGTTCCTCCTTCTG CCATGACGGATTGGAAG

LEFT PRIMER CATGACGTTGGAGAGAAGAT RIGHT PRIMER TAATGCGAATGGTTCTGGTT PRODUCT SIZE: 158

\section{G1_Unigene_BMK.9401 p6 (CCAAAC)4 24126149}

T C C C T C C C C C T A A C C A T C T C C C T T A T T T C T T CTCСССТTCССТTAATTCTTTCTTTATAAATTGAAC TACCATCCAATTTACTTCTCCCACCTCATTAATTAAT TAATTTCCCCCAAAACCAGAACCAAACCCAAACC CAAACCCAAACTCAATATATATTATCATGCCATCACC C A C C A C A A T C A T A A C C A T A A C A C C C C G T C C A C C T C C T G C C C T GC T C C A A T T T T C C C A C CAAATCGCCCTCATTTCCCGCCAAGAAATCTGTTTAC TACAAAAACCATCCTCCACAACCTTAGACCAA CAA T GGCAAA T C T T T C T GGGT T CGGT T T CA G TATTGGGTCCCGACTGGTGGCTTCTTCCGGTACTAG TGGCAGTGTTGCCGATGATGAGGTGCGCACCCTG ATTGAGTATGCCTCCGGCGAGGGAGGTGCCACTG TAGGAGATGATCTGGTGCTTTTGTTCCACCACTTA CAGTACGCCTGCAAGAGAATCGCAGCTCTTGTAG CTTCTCCTTTCAATTCCAGCCTTGGAAAGCACAAGGG
TCTCGCTGGCGGCGGCGCTTCGGCTGCTGGGTCAGG CCGGGATGCACCGAAGCCTCTCGATATCGTTTCG AATGAAATCATCTTGTCATCTCTTAAAAATTCTGG AAAAGTAGCCGTCATGGCTTCAGAAGAAGATG ATACTCCAATTTGGATAACTGATGATGGCCCATTTG TGGTGGTAACGGATCCTCTAGATGGTTCTCGAAA TATTGATGCATCCATTCCCACTGGAACCATTTTTGGG ATTTATAAACGCCTTCCGGAGCTGGATCATCTAC CAAAGGAGGAGAAGGCTTTGCTGAATTCACTCCAG A A GGGAACT A A T C T T G T T C T GCCGGCTA T G TTCTTTATTCATCTGCCACAATATTCTGCACCAG CT T TGGT TCAGGAACA T T TGCAT TCACGCTGG ATCATTCAACAGGAGATTTCATTCTCACACACC CAAGCTTAAAAATTCCTCCTCGAGGGCAAATA TATTCTGTAAATGATGCTCGATATTTTGACTGG CCCAACGGATTAAGGCAATACATAGACACTGTGAG ACAAGGGAAAGGCAGATACCCCAAGAAGTACTCTG CACGT TA T A T C T GT TCTCTGGTAGCAGAT T T T CATCGAACTCTACTGTATGGTGGAGTGACAATG AATCCAAGGGACCACCTTCGTCTGGTTTACGAAG C C A A C C C T C T T A G T T T T C T GG T GGA GCA A G CCGGGGGCAAAGGTTCTGATGGTAAAGGTAG A A T TCTA T C GA T T CA GCCA G T CA A C T GCAC CAAAGGCTGCCTCTGTTCCTGGGGAGTTTGGAGG ACATGGAAGAGTTAGAGAGTTACGGAGATGTGCAA CAGAAAGTGAACCCGGGATACGAGGTTTGACAA CATCAATAGGCTGATCTCACAAAATCCAGTGGATA TA TCGTCTGAGTAAAACCTGGGAATGGCTTAT TTTCTTGGTCACAAAGGTCTTAACTGTGATTCTG AAGCTGTGTGTGATTCCTCGTTTCCTCGGAAAC TAAATGTCATGAAGAGTGTCGCTGTTAAAAAC CAGGTGACATGAATATCTTCTGTATGTGATTTAT CA T G T A A A G A A G A C T A G T T T G T A T GA T A T G TTCTTGTAATTAAGAAGTCCTGTTTGAGCAAAAAG TTGCCTAGAATTGATGACTCTTTCTTTATCTCATTG AAGTGCAGCAGGTAAATGATATTTGGCTAAAGTAT TAGTGAATGAAATAAATTCATGAGGAA

LEFT PRIMER TTCTTCTCCCCTTCCCTTAA RIGHT PRIMER GTAAACAGATTTCTTGGCGG PRODUCT SIZE: 245

55. G1_Unigene_BMK.9465 p6 (TTCTGG)4 24488511 TGAAGTTCGTAAGTCTCGCCGCTACTTCCCTCTTCG CCGCCTCTTCTTACGCTATCGAAGTCGCTTGTCGTG TCAACGGCGTCCAGCAATCTGTTGTTGACTTGG A C A C C G G T A A C T G T C C A T T C C C A A T C C C A G CTAACTTGCCTGTGGTCTTTGACTTCGTCTCCGCTG ACAACTATATCGTTGACGCATACTACGCTCTTGTCG ACGGACAGAAGCACTGGAATGACATTGGCAATG CTGGCAGAACCATTGAAATTCCTGCTCTTTACTTG TCGACCTATGGTGACGCGCCACTCTACCAAATCG CTTTGAGCCAGACTCCTGCCATCAACTCTACCTCTG C C T T G A G A G C C A G A T T C A A C T C T A A G C T T G TCTCCAAGAGAGATGCCAGATCTGACTTGATTGCTTA CATTGAGTCCTTGACCGGTACTGCAGTTGCCGTCG CTTCCGTTGGTACTCCAGATGCTGCTTCTTCTAGTG CTTCTTCTGGTTCTGGTTCTGGTTCTGGCGCTGCTGG T T C C T C C A C T G C T G G C T C T G G T G C T G C T G G TTCTTCCACTGCTGCTGGTGCCACTTCTGAGTCCG C C GC T GC T G G T A C T A C T G G T C A A T C T A C C T C CACCTCCACTTACGTGTCGACCAAGCTCATCACCAT 
CACGTCTTGCTCCGACAACAAGTGCTCCGCCACCG CTGTTTCTGCAACTGAGGGCTTGACTACTGCCACCG TCAACGGAGAGACCACCGTGTACAGCACCTGGTG CCCAGTTACCTCGACCTCTGCTGCCGCCGCCTCTAC CACCACTGCCGTGACCACCAAGATCATCACCAT CACTTCTTGCTCCGACAACAAGTGCTCTGCCACCG CCGTTCCAGCTACTGAGGCCCCAACTACTGTCAC CACCAACGGTCAGACCACTGTCTACAC

LEFT PRIMER GATGCCAGATCTGACTTGAT RIGHT PRIMER GATTGACCAGTAGTACCAGC PRODUCT SIZE: 224

\section{G1_Unigene_BMK.9873 p5 (GAATT) 420500519}

TGGGGTCAGGTGACAAAGCCAAGGTTTGTCAATTTTC TATCCA T CATATGTTGTTGGCTTGTAATT TAG TTCATGTACGTGGCGCATATATGTTTCTAAATGTG CATGTTGTTGAGTTGATTTATCATTTAAGACAAAG AAACTATTGTCAAAATCTGATGCGCCTGTCGTATAT TAGATTTGGCTATCTGATATGAGTTCTAGACACTTC CATTCCAGTTGTACTTTTAATTCGTTTGCCATG TATCGTATGGGGGGTGGGGTGGGGGTTAACTTT TATTTCTTTTTTTGTATGCTGCCCTTAATGTGTTTG AATGTAGAAAAGTAATTTGTCAAAGACGGCTCCG AGTTCAAATGGAATGGAATGCCGCTGCACCTTG ACGACCGGACCGGATGAAGTGGTACTTATGCCAT CAGCAGCCGCAGCCCCT TTCTTTCTTTGCCAC CTTTCTCTCGTGTGCATGTAGGAGCCAGCCAGCCGG CCGCAGACCAAAACATGGAATTGAATTGAATTG AATTGGGAGTCTCATATTTCCCTTGCCTTTATTATT TATTGAAATTAATTCAAACCCAACTGAAAAGTAAC GATGTGATATTCCACATGGGGTATTTTATTTGACCG CGAGTCGGCAGTGGAATTATTCCCGAAACAAA TAAGGACCTTTGTTGTATTCTACAGTTGATATATT GGCATATTTTCG

LEFT PRIMER TTTCTCTCGTGTGCATGTAG RIGHT PRIMER AATAATTCCACTGCCGACTC PRODUCT SIZE: 196

\section{G1_Unigene_BMK.11082 p4 (AAAT)5 20102121}

CTAGTGAAAACTGCGGTAGCCGGAAATCAATGGAG ACTCTGGAACTCATACTTGAATACTTATTCAGAGAAT TATTGGAATTAAAGAGTCTTCCTCTCAGAAAATAAA TAAATAAATAAATGTAGTGATCAAATGAGTAATC TTCTGTGTTTTAAAGTCCCTTAAGCTGGTCAATGAG CCCGGTTAATGTTGTCCATTTCTCAATTGCACTGG CAGAAGACACAAATGCAAGCTTGGAGGACTCTACAT CATTCCGCGTAACTGATGATATCAATGAAGCTAGTG A GGAGT T GAAT GT A T C CACC T CA G T C T T C T C TTCAGGGCTAGAGCTTAGCTTATTCAAAGCGAGG TTCGCCTTCTGAACCCAATCTGTGTCAGTGCTGACTC C A A G A A C C G A A C T G G C T G C A G G C A A A T C G TTACTTTCGATGGCCTGGCCTGCTTTGCTTAGCCGG TACACAAGT TCC T GCA GATA T CC T GT T TC T T TCTTTGATGAGCTGAGAACTCCTTGCCTTTCAAG CCTCTTCTTCCTGTCCTTCTTAACCTTCTCCAACAG TTCCTCATCATCATCAGCTTCCAGAATTGCTGCAT TAGAACTGTTGAAATCTTTGCCGACCAATGAG AAGGCAAAGCTGGTGAGAAAGCAGATGGACAGG CTTCTTTTGGTGAGGATTGGAGCAGGTGAGTGTGG CTCTTCATTCAGGGCCTTACAAAAAGTCAATCC TCTCCTTGTACTTGTTTTTTGGACATGAAGTAATG
TCGGTGGTGTTGTCAGTGAAGAAGACGTAACAG AAGATGCCGAGGAGGAAAGGGTTGAAGGGAGG AAGGATTTTGCAGTGGAGAGAATGGCTGTGG CCATTTTCTTTGATTGTTTTTTTTACCTTATTTTTT TCTGATGATTTTTTTGGTCGGAAAATTCAGAGG TATTGAATTGACATGTTAGAACTTTTGAGGATAAG

LEFT PRIMER CGGAAATCAATGGAGACTCT RIGHT PRIMER AAGCTTGCATTTGTGTCTTC PRODUCT SIZE: 215

\section{G1_Unigene_BMK.11093 p3 (CTC)7 2111981218} CCTGAACCTGTCTTTAGCATTAACCATCAATGTGTAG ATGATGTACATTTGGTAACAAATCCTTGGCCAAA TAAAAAATAAAAAATAAAAGTGAAATCTTTAGTACA TACAAA TCA T T GAAAGGAA TAT AAAAGAACG CTTCTTTAGTAAATATATAAAATATACCAGATAACG AAATTCACTCCACACGAAATAACGGAATTCACTCCA CACGAAATCACAAAAACCAATCTCATATCCTCAG CAGCTAACACAAAATGAAGCTCTAAATCTCAAACAC TATTTAGATTTAGGGTTGGCTGTGCAACCTTCATGG CTCTCGGTTACACCAGCGGTAATGCTTCGTTTCTTG AATTATCTATCAGATCTTCGGTGCAGAATTTCGAAG AGTCCACAGCAACTCTTCCATGAAGTCGGCTG CAGGGGGCGTCCGGCGACAGGTACAATGGACGG ATTTCCTGTGCTTTCGGGGACCTCAGCCTTGCCATG ATCTTGTCCGGCATCAGCTGATTCGACTGCAGG AACCTCTTCCTTGTAAGCAGTACCCGATGACTCCC CAGTTTGCTCTTCGAAACCAGTAACTTTGTTTCCTCG ATCCCCTTTGACACAATATCTGCAACAGCAGCAGG AACCGCTTCCTTCTCATTGGAACAAGGCACTTCTG CTTCTTCAGTTTCTTTCGACACCAGATCGGTTTCG ACTAGAGAAACCCCATTACTCTCTTCCACATATG ACAATGTTTTTTCTTCTGTCTGTTTCGACACCTCCT CCGTGATAGCTGGCGAAGATTCACCGGTCTCATC CAAAGACACCGCAACTTTCTCCTCCACCTCCTC CACCTCCTCCACCTTGGTT TCCACTACAACAC CACCTTGATCCAAATGATTGTACACAGATTCAACCG T C T C A A C A A C C G A G A C A A C A A C T T C T T C G G TCTCCTCCACTTCCCCTGACTCATTCGCCACTCGAG CACCTTCCGCCTCTTCATCCGAACTATTACTTG AACTCGAACTTGAATAATCAGCATCATCGCGGG TTTCCTTTGCCGGCTTGGAACAGTCATCGATGGCTA TATCCCTCACAGATTCAACCACAGGTTTTGAGTCG C C A A C A T C C T C C G G T T G A A C A T T T T C T GC G CCCTCCAACTTCCCATTCAAAGAATCCGCCATTTCG TTAAGATCTGCAGAAACTTTCTGCTCCTCCTCC TCCTCCTCCTCTGCAACTCTCTCTCTCTCCCCTCT CAAGCAACACAGACA GCCACCAGCTCTCTCT TTCTCTCTCTGTGGAAGTTTTGACAGGGACAATG ACGGCGGTCTCCGCTGACGTCACGAAGCTAACGAAG CGTGAGAATATTAGTGACATGGATGCTTCTTTGCCCG CCAATGGGGCTTTTAACTGCGAGAGCTTCCTCAGCG CTCTCGATTTCCCAATGGAAGACATTGATGACAGCG TCGAAGATAACGATTGGGATACCAAGTTCCAAGACA TAGAGCCACCGCCTAACATTGATCAGTTGCTTGG TTCAGGGATTTTCAGCTATGATGACAAAAAGAAACA TACGAACCTCTCCACTCCGTATGGACATATGTATCAG CGACCGCAATTCCCAAGTGTTACTTGTGAAAC CATCCGCAGCAGAGTTAGCCTTCGCAAAGATG ACCTTGAAAGCATTGGCTCTTGTGCTGCGCAATTTG AGCCCAAATTTGAAAAGCGCACCCGAAGCAAG 
CTTTCACGAAGCAGGCGTGCAAACCCAATTATTTT C A G T A C A C A G T T C A T C C T C A G T A C C T C A T C CAATTCСTCTGCCTCTGAAAATTTATGTCGTTGGG ATGCTTGTGAATTTAACTTAGAAGCCTCTCGCACAG AGGACACATCAAACACTGCCAAAAGAAAACAGAAG AAGAAAATGGGAAAGCTCTCCATGGACGAAGCAGG TGAGAGTCGGGAGACTAAAAGATGCACACATTG TCAAGTGATGAAGACCCCTCAGTGGAGGGAAGGG CCACTAGGACCGAAGACCCTCTGCAATGCATG TGGGGTTCGGTACAGGTCTGGCCGCCTCCTCCCAGAG TACCGCCCTGCAGCAAGCCCTACATTCATCGCATCAG TGCATTCAAACTCGCACAAGAAGGTGATCGAGCTG AGAAGCAAGTCTTGCCAGGGGGCGAGCATGGGG ATTTTGTCGTCGGGACAGTAGGAAATTCAGATGG CAGTAGTAGTGGCAGGGGTAGTCATTTATTTCATTA TATCTGGAAAATGAGAAGAGTCTTCGTAGTTGAG ATAACTTTCTTTTTGTCTCTGTTTTATTGCTTTCTG TTCTAATTTATTTGATGTGTTCTTTGTGTACATGATGG AATAAGATCGTAGAAAGCAGTCGTTACCTCTG TCAAGGA A A A A C T A A A G ACAAA A GAA CAAACGAACTAGCTCTTGATCTCCGAGTTCGTTCGTG AGGGTCGACTTGTGATGTCCCAG

LEFT PRIMER CTCACAGATTCAACCACAGG RIGHT PRIMER TTGTCCCTGTCAAAACTTCC PRODUCT SIZE: 227

\section{G1_Unigene_BMK.11099 p5 (TAGAC)5 2518311855} GCAGCATCGATAATTATTCCACTCTCACTTCCACCG CTCGCAAAATCCCTACAACCCTAAACCCTAGGG T T T T T CA GA GCA GCCA GCC T C CGC C C T C T G TTCTTTGTTCTTCCCATAGTTCTTATCGAAACCC TATCTTAATCTTCCCAATGGGCAAATCAAGCAAG AAATCCGCCACCAAAGTTGAGGCTCCCGCTGTGG TTGTTCCAGCTCCTAAGTCTGGAAAGAAAGGTAAG AGAGATGCCGAGAACGAAATAGAGAAGGTTGTG AGCGCAAAGAAGCAGAAGATTGACGAGGGTGTAG CGCAGGCCATTCAGAAGAAGAAAGTTGAAGCAAAG ACCCAGAAGAAGAAAGTGGAAACTAGTAGTTCCG AGGAGTCTGATGTGTCTTCTGACTCTGACGTGAAGG C T C C TAAA T C T A A G T A GCTGCTAA GAA T GG CCCAACTGCTGCTATAAAGAAGAAAGATGTCTCAA CTGAAAGCTCAGATGAGAGTAGCGATGATTCGG AAGATGATGCTAAAACCCCTGCAAAGGTTACTG CCCAAAA GCCAGCTGCAGCTGCTAAGAAAGG CCCATCAGTGCCTGTTAAGAAGGCAGACACTAG CAGCTCTGAGTCTGAAGAGAGCTCTGAATCTGAAG A A GA GGA A G T T G T GAAAA A A G A T C T GC T G T A A T C C C T A A A G G A A A G G C G T T T C A A G CTCCTCTGATGATAGTTCAGAGGAGGATTCTGATG A T A G T T C A G A G G A A G T G A A G G G T A C C A CAAAAACTGTTGCCCCTGCTGCTAAGAGTGTTCAG CCATCCAAAAAGGATGAGGATTCAAGTGACTCTG A T GA T GA T A T GGA GGA T G T T GACTCTGA T A G TTCAGAGGAGGAACCTCCAAAGACAGAGAAAG TAAAGCCTTCTCAAGTTTCAAAGCAAGATAGCAG TGAGTCAGAGGAGGAATCCAGTTCTGACGAAGAAG A G G A A G A T G A A C C T G C A A A G A C T C C C A A G AAAAAGGACACTGATGTGAAGATGGTAGATGCCG A G T C T GA G A A G A A GC T C C C A A T A C C C C A G CTACTCCTGACGTATCGACTTCTAAGACGCTGTTTG CTGGAAACCTGAACTTCCGTATTGAGGAACACG
ATTTGAGGAATTTCTTCAAAGATGCTGGTGAAGTTG TTGATATCCGTTTTGCCTCAGATCCAGAAGGGAAG TTCAAGGGCTTTGGACATGTTGAGTTCGCCACAG CTGAAGCAGCAAGGAAGGCTCTTGAACTTAATGG T T TAGATT T GCT TGGTCGTGATATCAGACT TG ATCTGGCTCGTGAAAGGGGTGAAAGGGGTGCTTA TACTCCCACCGGAAGAGAGAGCAACTCATATCAG AAGGGACCACGCGGTGCGTCCAAAACAATATTTG TTCGAGGTTTTGACAGATCCCTCGGGGAGGATGAG ATCAGGAGCTCCTTAAAAGAAGCTTTTAGTTCTTG TGGTGAGATAACAAGGGTGTCCATCCCCAAAG ATTATGAGACTGGCGCCTCTAAAGGGATGGCTTA CATGGACTTCTCGGATGACGCCAGCTTCAACAAAG CTTTAGAATTTAATGGACATGAATTTGGAGACGG TTACTTGCAGGTGGAAGAGGCAAAACCAAAAGGTG A T T T CGG T A C T C C CAGGAGTAA T GA CGGGGG TTATTCCAATAATCGTGGTAGAGGACGTTTTAGCAG CAGCAGCAGGGGGGGTAGGGATGGTGGTGGACG TGGTTTCAGGGATGGTGTACGAGGCAGAGGACG T G G A A A C A A A C C A A A C C T A G C T G C T C C T G G AACAGGGAAGAAAACCACCTTCGACGACGAGTAG A C T A G A C T A G A C T A G A C T A G A C T T G T G A G TAATCGGTCGGCGGCCAAGGGAAATTGTGGAAG ACTTATGTTTGGCTCTTTTTAATTTTATCATATTT TATTATTTTAATTTTGAGAGGCATGTCTGGTTTTG AGCTTTACTTGGACATGAACTCGAACGCAAGAGTG AGTGAACCTACTATGGAGTTT T TGGATT TATG TCTTTAATTAATTTTGAGCGTTAATATGGTATTT TATTACTCAAATTATTTCGGATCTCCGGC

LEFT PRIMER GTGGTAGAGGACGTTTTAGC RIGHT PRIMER GTAGGTTCACTCACTCTTGC PRODUCT SIZE: 330

60. G1_Unigene_BMK.11165 p6 (AACCCT)3 1812311248 GACCCTTTCTCAAATAAAATGTTCTTAATTTCAATT CAAATGCAAAGGATCATGTTATCAATATCCAGTGTG A GACT T GG T T A A A T T T CAACCGT T C A C T T GG AAAAAAAAAACCGGGGGAGAATTTTCAATATAG ATAACCTACAATGATCCTTAAAAAGATGAATATG AGGGGCGGGGGGACTAGGAGTGCAGCTTGACAA CACGACATAACCTTTCAAAGAAGATAGGGCACATG TTTGTGCTTAAAACTATGTCATCCTTCATCCATTCT GGTGCTTCCCTCTCAAGCGTCCCCAAGGATGCGGTG CGATACAGAAGTTCTACTTCGTTACCAGCAGCCCTG TTCATCCATGTAAGATTGAAGGCATAACCACTTG ATTGATGCACAGCTGAGATGCATATTTCTTCAGCTTG ACTAACAGTAGAGAACTTTAAATCCACCATATACT CAACAAGCACTTGAAACACGCAGTTAACCTGG CCAATTGGTTTTGCACAACCTGGTCTTTCAATAG TTCTACACCATTGTGGCTCTTGAACAGTTACAAGAG CCCCACTAGAAGGCATAACTAAATCCTCTGCCTCAG A C T G G G A A T T C T T C C T A C G C T T A C T T G TCCTTTTTTTCTTTGAGAGATTTCCTGCTGCTCCTG A T A C T T G A C C A C T A G C A G G T G A C A C A G TCCA T T C T C T G T G T T GCTACC T T T C GA T G T G CTAAAAT T TCCCTCCTGT T GCAGCT T T TGGAG CСТCTCTACTTCATCAGAAAGTTTTTCATTTTTCCTG T TCTCTTCCAACAAATGATTCTGGTACTCGGTG CATTGTTCAGCTTTGGTGGACCTGCATTCAATTTG A TGGTT TCAAGA GCCCGTTATGTAGGTTATAG TAAAAATTGACTAATCGGAATTCCAATGGATCCG 
ACAGAACT TAGAAT T TCA TGCCTAATAGAAG CAACTTCACTTCTCAGATCACCAACTTCCGAGCG CAACCTCTCATTTTCCTTTCGCAAATGCTGAATA T A C T C C T C C GCA A C A G A T A C A T A A T GC T T G A ACT T TCCT TC T TGA TCT T TACCAATATCT TC CAACTCCGACCATTTCTTTGTCTTGATTTTCGAGTACT TATCGTAGAGTTTGGAGTAGAGAGCTTCTGTGGTCG ACATTTGTCAGAAGCCCTGACCAATTCTTCCTTTCG A T G A C C A G T A C G A T C G C T G A G C C C T C A G G CCTCCCCCAGGCGAAAACCCTAACCCTAACCC TAATCGCCCTGCTACTTGTAAACTGAGGAGGACG ACGACTCTGTGAGAGTCGGAGACTGAGGGGAG ACAAGGCGGGAAAGTTGGAGATGTCAAAAAGG ACACGGAGGGGAAGAACGCCAGCTACTCGGCTGATG T T T T T A A A A T T A C A T A A A C A T C C G C T G A T G TTCTTAAATTGTCTCGCAAGTTGATGAACG

\section{LEFT PRIMER TCTTCCTTTCGATGACCAGT} RIGHT PRIMER GTTCATCAACTTGCGAGACA PRODUCT SIZE: 265

\section{G1_Unigene_BMK.11242 p6 (CTTCTG)4 2424552478} GAGAGAGAGAGAGAGAGGAGAGAGAGCCCTCCCA CTGTTCTTCCCATATCCCTTTACCCTAGCTAGCCCACC TCAGGAATCTCCCTTCCTCTTCTCTGTTTCTTCTTC TCTTGAAACAGAGAGATTTTCACCATTTTAATTTTCGG TTTCCATTTGGGTTTTGTGGGGTTCAATTCATGAAA CAAAGATTAATTGCAGAGATATTGTAGAGAGAGATA C A C A C T C A C T G T A C GC A G A A A G A G A A G G A G ATAAAGGTAGTTAATACTTTCTGAGATCATGAAACA TAAGGATGGAAAACCAGGGCCCCAAGGTACCAGGG TTTTCCCCATGACAATATTGTTTGTTTTGCTATGCGGG T TT TCATTCTA TCTTGGTGGAATGTTCTGTTCTG AAAAGAACAAAATTGAGCCTGTCAAGGTCGAAGATG T C A C A G A G G C A G T T C A A T C A T C T C T C C A G TTAAAACCCGTTACCTTTGCTGAATGCAGCAGTG ACTATCAAGACTACACCCCATGCACGGATCCAAGG AGGTGGAGAAAGTATGGTGTTCATCGGCTTACTTT CATGGAACGGCACTGCCCTCCGGTATTGGAGAGG AAAGAATGCTTAGTTCCACCTCCAGATGGCTACAAG CCACCAATCAGATGGCCAAATAGCAGGGACGAATG TTGGTACAGGAATGTGCCATATGATTGGATTAACAAA CAGAAG T C CAA T CA GAA T TGGCTGA GAAAAG AAGGGGAGAAGTTTCTCтTтCСтGGTGGGGGAAC TATGTTTCCGAGAGGCGTTTCTGCATATGTCGATTTG ATGCAAGATCTTATTCCAGAAATGAAAGATGGGACCG TTCGAACTGCCATTGATACTGGGTGTGGGGTTGCAAG CTGGGGA GGTGA T T TGT TA GA TCGTGGGA T T T TAACTGTTTCTCTCGCTCCAAGAGATAATCATGAAG CTCAGGTTCAGTTTGCTTTGGAACGTGGAATTCCAG CAATTCTTGGCATCATTTCTACACAGAGACTTCC T T TCCCT TCTAACTCGTTTGATATGGCTCACTG CTCAAGATGCCTCATCCCATGGACGGAATTTGGTGG AATTTACCTCCT TGAAGTTCATCGTATACTCCG TCCTGGAGGTTTTTGGGTCCTGTCTGGTCCACCTG TCAACTACGAAAACCGCTGGCGTGGGTGGAACAC CACCGTAGAAGAGCAGAAATCAGATTATGAAAAG TTGCAAGACCTGCTAACTTCATTGTGCTTCAAATTG TACAACAAAAAGGATGATATTGCTGTTTGGCAG AAACTGTCAGACAGCAGTTGTTACAATAAACTTTCTG AGCCAGACACCTATCCTGCAAAGTGTGATGATAG CCTTGAACCAGATTCAGGATGGTACACTCCATTACG
CTCTTGTGTCGTAGTTCCAGACCCAAAGCTAAAAAAG TCAGCTTTGAAATCCATCCCTCAATGGCCAGAGCGG TTGCATGTTGCACCAGAGCGTATTTCAGATGTG CATGGCGGAAGTGCTAGTGCTTTCAATCATGATG ACAGCAAGTGGAGGGTTCGATTGAAGCAT TA CAAAAAGTTGCTCCCAGCAATTGGAACAGATAAG ATCAGAAATGTTATGGACATGAATACGGTATATGG AGGTTTTGCCGCGGCCACGATTGATTATCCCTTG TGGGTCATGAATGTGGTCTCTTCCTATGCTGTAAATA CACTACCTGTGGTCTATGATCGAGGCCTTATCGG AACTTACCATGATTGGTGTGAGGCGTTTTCTACT TATCCTCGTACTTACGATCTTCTACATCTTGATGG CCTCTTTACTGCAGAAAGCCACAGATGCGAAATG AAAAATGTGCTCTTAGAGATGGACCGAATCCTGCG CCCTAATGGCTACGCAATAGTTCGAGAATCCAG CTACTTTGTGGACGCTGTTGCGACTATTGCCAAAGGG ATGAGATGGGGATGCCGCAAAGAAGACACTGAG TATGGCGTTGAGAAGGAGAAAATACTGATATGCCAG AAAAAACTCTGGTATTCATCGAACCGGACTTCCAG ATGATGGCCATCCATAGAAACCCAGAACCAAGTCG A T T TCT TGGAGGGACGGAAATAGGAATAGAAG CAAAAGAAGCAAGTTCAATAGCATTCACAGAGAAT TAGTTCATTGTTTAAGACACGAATACTCAAATTTG TCGTTGAAGACTCGGTTACCCAATAAGGTGGTTTAAT CAACTGATAGGGCGTTGCGTATAGATATATTCTTTA TAATGTGTCAACATGTACTCGGTGTAATTTCCTTTTCG T T T T C C C T G T T T C C C GA T T T T CA T A A T T T GCG TTCTTTTCGTGTCAAGCTGTAATCGGATGATTTGAATA CATGCAAGGAAGTGTATGTACCAAAAGAAGAAG AAAGAAAAAGAGAAGGAGCAAAGGTTTCTTTG TTTTCTTAAATGAAAATCTTCTGCTTCTGCTTCTG CTTCTGCTTCGCCTGTGACGCCTTTCTATTTGATTGTG AAGAAGATGGGCTATTAGAAACAATCTGCTTCGACGG AGAGATGTGAACAAGTTCATGCCTTAATTCAATCTG A G G A T G A G C T T T A C A G A A G G A T G A G A T T G CATCCTCGGTTAAGAGCCAACAACCACTCAGGTCCAT CATCCTTAGGGTCAATGGAGGTTTATATGAATTGAG TCCGGAATTGGTCAGAACACCATCATGAACGCAAAG ATTTGTGAGCTTTGGTATAGATGATGTGTAGTAAAG CGAGTTGTCTGTAAGAGACGCACT T T TCAGAG TTAAATCGGTTAACTCATGGAAGCTCTTTAAAGGG TCAAGAGCTGCATCCGTAACTTTTGTATCCTTCAAATT CAACTTTTCTAAGTGTCGGAGACCTTGAAGTGCTG ACAGCGTGAGAACTGGACATGAATCAGGTTCAACC TGGTGAATGACACCTGAGAAAGGAAAAAACACA TAATTATGTCACAAACTCCTGCAGCTGAAATTTG TTCACTTCACTGTATGCAGTGATTAGAAAATCACTG CATAATGATGGACAAAACCAGAGCTCTAGCATTTG TATTTATCCATACCTTTAATGTTCGTATTGCTCAAG TCAATAACTTTCACAAATGGCATCATGCTAATATATG AAATGGCAACATCATCAACCGACGTATGAGATACTG ACAAGAACTCAAGATTGGGTACATGTCCAGTG ACAACTCCAACTCCAGCAGAGCTGACTCTGGTGCGG CTAAGATTTAGATATTTCAAATTTACTCCAATACGTG CAATTAGTTCAAGCCAATCATCCCCTATTGTACTAG AGCTAAGATCCAAGTGTTCCAGAGCATTTAGATGAG ACAAAAAGTAGAATTTCTGAAGCGTGGAGTTGG ATAAATCCAAAAAGGTCAAAAAAGATTTTTCAATA TAATGGAATGCTTCAAACTCGTCCCCAAACGTAG C T C C A G A A G C A A C A A G C T T A G C C A A A G G A G CTCTAATTGTACAATTAAAGCTGGTGAATGGCACCTG AAGTAGAGAATTAATTGTACAATTACTCATGTTTAG 
ACATTGGAGgGATGACAAATTTGGCAACTTCGTCA C A C T G G T C C A A G C C A G A T T GA G GA A A C T CAACTTGGGAAACGCTTGAAGAAGAGCAG CACCTTTGTCAGATATTTTACTCCCCCAAAG ATCAAGGTACCGCAGTTTAGGAAGTACCTGAAG ACAACTCAGTGCTTGATCAGTAACAGGCAAACCACC CAAGTCCAGGAGAGACAAGTTTCTCAGCGAAGCG A GAAGCATAACCCCGTTTGCCGACACACCT G TTTCAGAAATGCACAACTTTTCCAATGTTGTAATAG ATAGAAGATGCCTAATCCCAGCATCAGTAACCTTGG AGCATCTCGAAAAGTCCAACTCCCTCAAACTCT CCATCCCAGTAATAGGCCAAAGAGCAGAGGCAGTG AGTCTACGGCAACCAGAAACGTTCAAAGATCG CAAGTACCGAAATGCCCCTAGGTATGCAATC CATTCGGCGTCAACGGAGCTCTCCCCTCGCAG ATCAACCTCCTCCACGCTGTTTTTGAATAATTCGAG TAAGGAAGGGAAGAGGAGGCGGCGGGAGATGAGG CGGCGAAGGAGAGAGTCGGCGAGCTGAGGAGG CAA GC GC T C GA G A C T C C G T C GCT GC C T C C G CCATTTCTCGACGCTGTCTCTGCTTTCAGAGGCGG C T T G T A T GCA GA GC T C C A C C A A T T C C A C C G CCATCTCTCTCTCTCTTCTTTTCCCTCTTTGCCTTTTG CGTTTGTG

LEFT PRIMER CCCTGTTTCCCGATTTTCAT RIGHT PRIMER CTTGTTCACATCTCTCCGTC PRODUCT SIZE: 256

\section{G1_Unigene_BMK.11339 1 p6 (AGCCGG)3 18542559}

GGACCGCGTCAACTGCCCCTTCTATTTCAAGATCGG CGCCTGCCGCCACGGTGACCGCTGCTCTCGCCTG CACAACCGCCCGACCATCAGTCCCACGCTGCTTCTG TCCAACATGTACCAGCGGCCCGACATGATCACCCC CGGCGTCGACTCCGAGGGCCAGCCCCTCGACCCGCG CAAGATCCAAGAGCATTTCGAGGACTTCTACGAGG ACATCTTCGAGGAGCTCACCAAGTTCGGCGGGATCG AGAGCCTCAACGTCTGCGACAACCTCGCCGATCA CATGATCGGCAACGTATATGTCCAGTTCAAGGAGG AGGATCAGGCCGCCGCCGCTCTTCAGGCGCTG CAGGGTCGCTTCTACAATGGTCGCCCCATAATCGCCG ACTTCTCGCCCGTGACCGACTTTCGCGAAGCCACGTG CCGCCAGTTCGAGGAGAACAGCTGTAACCGAGGGGG ATATTGTAATTTCATGCACGTCAAGCTGATTGGG A GGGAGCTCAGAAGGAGGCT T T T CGGGAGG TACCGGGGCTACAGAATAAGCCGGAGCCGGAGCCGG AGTTTGAGCCCAAGGCATCGGAGCAGGGACTACG ATAAGTACGATAAGAGAGAGAGGGACTATCGGG ACCGTGATTATCGCGGGAATGGGCGACGGAGCAAGG ACAGGTACGACAGGGACCGGGACAGAGACGGTGGG AGGAGAAGACACGGCAGCAGTCCGAGGCGGAG CAGGAGCAGGAGCCCCGTGGTGGTGAGGGAAGG CAGCGAGGAACGCCGAGCGAGGATTGAGCAGTGG AATCGAGAAAGGGAGGAGAAGCAGTTAGTGATGTG AAAGCGCATTGGCATTGGGATTTTTCACATGGATTGG TTGATTTGATGGCTAGTAGTGGCGCGTAAAGCGCTTG TTTGCGGAGACACTAGTTTTGATGAATTCCACTGCG AATGCAGTACGTATATCACACAGCTAGGTAG ATATTTTGTTGTTTTTTTTTTCCTTTCTAATTT TACAGGTCGTTGGTATTTATAGCATCTTGTTCGTTA TAGATTCTTAGATCGGGGGTTTGTTCTGTGATCTAC TAATCATAAGTTGAGAAGAAGCCGGGTTCAATGATG AATCTAAATTGAACTCTCAGCACACCAATGATATG
TTGGGATAGGTTAGCATTTGTTTTCTTTCTTTGTTAG AGTATCATTTGTGTATCACTTTCTGGCTAGAAAG CATTGGTCCTGCTATTTTTAATGTTGCATTGTTCAG ATTTCCTCTCCTGATGTTTGCTCAAGTCAGATTTTT CAGCTTCTTGCCCAGTCTTTTATCTAATAATAATTCTG CTTATGATTTAGGGTCTGATACAGTAGCTCTGAG AATGGTGTAGGTGAACAGGAAAACTGCTCAGAAA CAGGTCATATGGCGAGTTGACGGATTGATCTAACTAG CTATGGTGTAGGCCAACTCTAGGGGCTCCTTTGGTTTG TCGGCAATAGATGGTTGTTACATAAATATGCTATCAG A T TAATCGGTCT A GCA A ACA GTGATCACTAG TTTCATATTTGACTTAATGGTTTCTAGGTGGTAG TTTTTTGGCCGCAGGAGAAAAAAACCATAATATTAAG CTCTAATGAAAGATGAGCAATTGATTTGATTTATTCAA CAGTGCGCTGGTCAGGAAGCTGAAAACAATGAGTGAG AGGTTTGCAGGGTTGACAGATGCCTTGGTGTTTGGTG TGGATTCTCCTTTGTATTTTTCTGCAAGTCTTTTGAATG A T GA TA T T T GA T A T T T GA T A T T T GACT GA G TTTTCCTCTCTTAGGTGAAGGTGTTGTTGATAG CATTTCTGAAGCTCGACCGCAAATTTAGGCTATTCGGG TAATCTGTGTGTCGTGGACAAGCAGATAGCAGTTA CAGGTTGAAATTAGAAAGGCAGGGCTTAGGATAATG AGTATTGTAGGGCAGGATTTGGTTTGTCTGGGATG ATTGATCGTTCGAACTTCGGTCGATCATGTGCAAGTG CAAGTGCAATTGCAAATGCAATGCTGTTTGTTGATGG CCTTATAATGTTGTTGGTAGTGAGTGTTGAAGAG TTTAACTTACGAAGATGATAAAACGATTTCCTCTTTTG AATCTCCAACGTACTTTTGCATGTAGGCAGGCTGAG ATTTGTTGTGATTTGGCCCTTCTTCCACTTGCGGG ATTGAACGGTTAGCAAATCGGGGTTTGTTTCTTTG AGGGGAAGTCCGATAAAATTGAAACGATCCG

\section{LEFT PRIMER AATTTCATGCACGTCAAGCT} RIGHT PRIMER TCGTACCTGTCCTTGCTC PRODUCT SIZE: 206

\section{G1_Unigene_BMK.11347 p3 (ATT)6 18711728}

TGTATATATATTATTCATTGAATTCATTACCTTAAG AATACAAAAATTACATTGTGTGAGTTGCATTTTGTGG CAAAGTTCAAAAGTTTCGATATGAATCAAAATCTATG AGAATTTAATAGTATTTATGCAAGATATCAAAG ATCAACTAACATTATCAATGTTTAGCACTATCCAT TATGAGTTTCATGGCTTCTTCCTACTCTCTCCCTGAGG AATTGTGGCTGTTTGAGTAAGGTTTGTACTTTG ATTTCGCAATCTTGTTACTCTCTCTTAGTAATG CTTTTGTCTTATATTTGATAAGACTAATGTACTTTG CTATTTGAAAAATCTCTGTTGCAAGTGAAACC TATCTGCTTGAAAGCTGAAACATTAGCAGACTCAGAG TTCATTTTGAGTTAAAAAAAGGGATCTCGATTGTGTG TCAAGATTTCAAAGAGACGAACTTTAAAAAGACAG ATTAACAAGCCTTACATTTGAACAAAGGGATCTTTTT CAAGATTTTAAAGAGACGGTTCAACGAAACTTA TATCTGAATGATGGGATGTAGGCCAGTTGATTTTG ATGTTCAATTTGTCTCTTCATTTTACACTTGAATTTG ATGTTTTCCTTTAGAAGCTAAATATTTCCTTAAT TAAAACTCAACTTGTAAAACCCCCAAAAGCCAAAG ATTAAGCTAAATATTTCCTTAAGTTGATGATTTTCATC TGATTATTATTATTATTATTTGTTAAATTCCTTTG ATTATTTTTGTTAATGATGAACTCCCTTTCAT TAATTTGTTGCTTTGTTTGCCGCCAAAGCTAAA TAAAAACAGATTAAAAATATACAAAAACAAGAAA TATGAAAGTGAAGGGCTTATCCGTAGTGACATGG 
AAAGGTAAAGAACTGGAGGACGTTGACCACTTCC CAACCCAACCTGGTGAATACGTCAGGGGCCTCTG ATATTTCCCTTTCAATTGCCTGAAGTTTGGCTTAG AAATTGTGCAATTCTGGACCTATGTTCGTACTCTTG A T T A T T T G C T T A C C A T T T C G G G T A A T C A TACTTCCTTGGCTTGTTGCTTCCACGAAAATAAATAC CATTCACTCATGACTGGCTTTTAATTTTCTTTTTCA CA T T GGGGA T T T T A T T C T C TCA T G T GGG T T GG ATTTTTCGAGATTTTTTAGTATTGATGTATTTTATA CACTTACGTTTTAATCGTTTGATTCTTTGTCACGTATC CAGTAACCAATATTAATTGACAAAAAAAAAAAGGG TGTGGATGGACGGAGGCAATGAAATTATGTAAG ATAGGGTTATGATGGATTTTTTTCGTTCTCAATTATAA CAAAAGGTTAAAGTCGCCTGTCTAAAGAAAATATA TAGCTCTGTCATGAGGTATGGTGGTAATCTATGGC CTGTTTGGAATTGCATTTGGAAGACGAAGAGTGTTT TTGACAACTAAAAATTTTTTTT

\section{LEFT PRIMER AACTCAACTTGTAAAACCCC} RIGHT PRIMER CATGTCACTACGGATAAGCC PRODUCT SIZE: 237

\section{G1_Unigene_BMK.11454 p3 (AGG)6 18143160}

AGAGAGAGAGGGAGAGAGAGAGAGGCTGTGAAG CTCCCAGCTTTCTGTGTGAGAACCAATTTTGCAA AAAACCCAAAGAATTTGCAGTTGAGAGAGAGACAG AGAGAGGCGGAGATGGAGGAAGAGCAGCAGCAGAG TATTAAGGAGGAGGAGGAGGAGGAGCGTCCAGTG AGAGTATATGCAGACGGAATTTATGATCTCTTT CACTTCGGCCATGCCCGTTCTCTCGAGCAAGCCAAG AAGCTGTTCCCCAATACGTACCTGCTTGTTGGCTG CTGTGGTGATGAAATTACCCACAATTATAAGGG AAAAACTGTTATGACTGAGAAGGAGCGCTATG AATCGCTCCGCCATTGCAGATGGGTGGACGAAG TTATCCCTGATGCACCATGGGTGCTCTCGCAAGAG TTTCTTGACAAGCATAAGATTGACTTTGTGGCACATG ACTCTCTTCCCTATGCTGATGCAAGTGGCTCTGG AAAGGATGTCTATGAATTTGTTAAGTCTGTTGGGAAG TTTAAAGAAACAAAGCGAACTGATGGGATCTCTA CATCGGATCTCATAATGAGGATAGTTAAAGATTA CAACCAGTATGTGATGCGTAATTTGGACCGTGGG TACACAAGAAAAGATCTAGGTGTCAGCTATGTG AGGGAAAAGCGACTGAGAGTGAACATGGGACTG AAAAGATTGCGTGAGAGGGTGAAGGAGCACCAAG AAAAAGTAGGAGAGAAGATACAAATAGTAACACAG ACTGCCACCATGCGTCGTAATGAATGGGTGGAG AATGCTGACCGGCTGGTTGCTGGATTTCTGGAGAGG TTTGAAGAAGGTTTCCATAAAATGGGAACAGCCA TAAGGGACCGAATTCAAAAGCGAATAAGGAAGCAG CAGTTCAGAGGGCTTTTATATGAAGAAGAAGATG ATGAATTCTATGATGACTCTGAAGAAGAAAACAAGG ATGAAGACGAGAACTGAAGTTGCTTCTGTTGCTG TCAATTAGGAGCTGAAATACTTGTCCGTTGTAT TAATGTAGAACTAACTAAGAAGTCGATAGCATTAG ATTCCTTGATTATATAACCTCAGTTTGTGAAACAG CCAAAATCAAGGCTTGGATGTTTAAATTCAATTATAG TATTCTGTCATTTTCGTTTCGGTAGGTGCTTGTTTG ATGTAGTTGACAGAAAATCTTTATGAAGATCACAG

LEFT PRIMER GTGTGAGAACCAATTTTGCA RIGHT PRIMER TACGTATTGGGGAACAGCTT PRODUCT SIZE: 214

\section{G1_Unigene_BMK.11485 p3 (CGT)6 1823992416}

CTTGGACCATCTTTGTTAAAATTACGGGTCGCGATCC CAACCATTTAGAATTCATTCGTGGCTCAAACAGG CCTATATCATGTCCTCTCACCAATGGAGTAAACTGG ACCTTATGCTCTGAGAATACTAATGTATACAGATTG C T T A A GGGGA G G A A T T C C C A T T T T T T C A A A AAAATGGGGACACGCTCCCCGCCTTTAGATTGG CTTTAATGAAATTACGTGGTTGAGATTAAAACACAGG CCGCATAATCTCAACCACACAATTTCATTAAAG TTAAATCCAACGGTGGGAAGCGTGTTCCCATTTTTT TAGAAAAATGGGGATTCCTGCCCTTAAGCAATCTG TTATTATAAATAGGCATGAATATTACACTGTTTTA CAAATAAACCAGATACAAATTTGAATCAAATTCGG CCTGCATATTGTGACGTGAGGTTTGAGTACCAACTAG CAACAGATGGAGAACAAGATCACTGACGAATAT CTTCGTCGGTCAGATATCGAATGTCGGAATTTCAG ATGTGAGGCCAGACATGGCCTGGTATTGTATCCGAG ACAATAACGTTCTATACGGATCCTTTTCTCTATT СТCССТCAAATAAGCAAGGCAATTCTCAACTTCTG ACTTCACTTGTAAATACAGTTCATGAGCTTTCTCCCTG TCTCGTAATTCCTGCTTCCTCCCTTCTGTTTCAATTGG TTTTCCAAAATAATAATAGAACCTGCCAGGAATT TTCGGTATGACTCCTGGCAGGTGTACATCCTGG TTTCCTACCTCCCCACCAACGCCCTTTCTCACTTTCG CAACCTCATCTGTTAACTCCTGTATAAAGTCCTTAAG CGGAGGAATTCTCATTTGGTCGTCATAATCTAAAAA TAG T T C A C C A A T A T C A T C T T C T C C A A C G GCG CCAAAAGGTACAATTTTGGCTCCAAATCTAGCCG CCAT TC T CACAAACTCCGACTGT TCTGGCCAG AATAACTTATATTCCTCCCCCTTCCGGTGAAGAG CCTCACGCAATCCACCTGGATAGAGTAGGACGTGAG ACTTGGTAGAGAGAAGTTTGTAAAAGTTTGCACCAG AGACAGGAACTGCACCCATAAGTCTGAATATGTCG AATGCTGATAGATCAGGAAACCTTCCTTCTCTCAATTT TATAAACATCATCGGATGTGCTATTCCTCGCATTAGG ATGTTTCTTTCCTCCATCATCTGAGTTACCATGGGAG CCAATTCAAACCCCAGTAACATGTGATAACCAACAAA CAATACAGGACCCTCAGAAGGAATCCCGGCAAGG CCCCTCACAATCTTCCCATCTGGTAAAGTTGAGAG CATCACTGGGCTAGTGACCGTGGTTAACAATCTG TTTGATTCAATTATATTTTTGACTTCTGATGGCGTTGG TGGTATGTAATCAGTAACAAAGTCATGGGCACCAG AACGACGATAGACACCAGTTGCCTTGATGACAGTTAC CAAATCAAAGGGATCTTCCAAGAAGAGGAAATGG CCGCTGTTAGCAAACATACGAATCTCACATTTTG CAAGGGTGTGTTTCAGTCTTTCTCCTTCCGCTAG ACTAGGTAACAACTGATCCTGTCCACTGGAAAGTAT CAGCGT T T GA GC T T T T ACAGCGTGGAGACGTG AATTGGCATATGGAGATGCTGATCTAATCATTTGCAG CTTCCACAGAAGTGTTTCTCTAGGTAAGATATCAG CAAGAACAGAGAGGTAAGATGCTGATACAAGAAG ATCACGTGATAGCTTCTCAACTGTCTGCGGCAGAGG AAGCCCAAGTCCCGTCTCCAAGTTACCCATCAG AAAACCTGTCATTGAGCTCAATATGTGAGGAAGGCCG AGAGAAAGTGAGTCAGGAATGACCTGCAGTAAAGG TATTAATGGTTGAAGCTGGGACTTGCTGAAGGACG TAGCTGGATTAGCCAAAATTAGAACAAGGTCAATAT CAGGGTTAAGGGCAGCCACAGAAAGCGCAAGG CATGCTCCAAGAGATTCTCCAACAAGATATATAGG CCTATTTGGTGCAAGACAATGCTGTGACCGAACTG TTCTTTCAACCAGCTTCACAAGGTCTGTGAATGTTG T TCGATCCT TGGCAGGGATATGCAAGCACCAG 
ACATCGAAAATCTTTCCAAGTTTCTGATGATG CCTTATGAGCCCAAGTCCAGTGCCATCAATTCCAGG CAAAAAGAGAAGCAGAGGAGAATCGTCCAAGCGGG ACCCGCCGCCCAAAGGAGAGAACCAGCGAGGAGG TCCACCGTCGGAGCTGATGAAATCCTTGGCTTGCTCG AAGTAATCCTTCAAGCTCAGGCCTTGCTCATCCG CATCGAACCTGT GCTCC TCCT GACGA GCCT G CCTCTTAGTCTCTGCAGCTGACGTCGTCGTCGTCG TCGTATGAGCTTCCTTCTCAGACGACCTGCCG CTTCCGATGTTCGCTGCTGTCAGAATCGTCGGAGTG TGGTCTGTTGACGCAGCGGCTAGCCTCCGCGACGG AATCGAGATCGGATTGAACTTTGATCTTCCGGATG AGGATGATGATGACGATGACGACGCCAGTTCCTG ACGGATAACGGATGGCAGGCGGGCGGGGAAAAG ACAAACTCCGGTGGAGGCCATGGACTAATGGAGG CAGCTGGCGGGACGGCCGAATCGGCCGACAGCTC CTGCTGGCGGTATTTAGAGATTTGAACGCTTAT TAGTGAAAGTTATTTTTCCATTTGAGGGATAAAG TCAAGTATTGAAATTAAA

LEFT PRIMER GGAGCTGATGAAATCCTTGG RIGHT PRIMER CGTCATCGTCATCATCATCC PRODUCT SIZE: 287

\section{G1_Unigene_BMK.11513 p3 (GGA)8 24145168} GAGACGCGCACCAAACACACCCAAACAAACGCAG AGACGCTGAGGGCTTGAAGGAATGGCTATGGCG AACAAATACGAACAAGAGGAGGAGGAGGTGCAGAG CTTTGGAAAGCGGCTGAAATGGAGCAGATACGAGG AGCCTCTGGAGGAGGAGGAGGAGGAGGAGGAAG AAAGTAGGAGTAGGAGACCATCAATAGTGTATGGTG AGGATGAGGGGAGAACAAGAGTGATGATGGTG AATAGTACTGCTGCTACTCCTACAGGGAGAAG ATCTGGTGCAGGTGGGTCTACGGCAGGGCCATGTTG TCAGGTAGATAATTGCACTGCTGACTTGAGTGATT TAAAGCAATACTACCGCCGCCACAAGGTCTGTGATG TTCATGCCAAGGCTCCGTCGATAGTCGTCGGTGG ATCCCGGCAGCGTTTTTGCCAGCAATGTAGCAGG TTTCATAGCCTATCAGAGTTTGATGACAGTAAAAGG AGTTGTCGCAGGCGATTAGCTGGACACAATGAGCGG CGTCGGAAAACGTCATCGGAATACCATGGACATGG AGGCACGTCTAATTAAGTAATTAGATATAGATA TAATCTTTTCACGTACATGCCTGTTTCCACTAGCAT ATGATATGCTCTCTCTCTTCTGTCAACCCTCAG C C T T GA T C T T GC A C CA G T GA A C T C T C T C T C TCTCTCTCTTAGTTGTATGTTTTTGGGGAAGAATTG CTTTGCATTGATCTGTAACCAAACAAATATGCATAG CTTCTTTGCCCGCACAATTTCCATCCTTAATGTTTG TTGTTCCTTTCTTGGCTGTAATTTGAAACAAACCTA CATTGTTTGATCTGT TCA GTTGATGATGATG ATTCTTGTTTTCTTCACATGCTTTAATATATCAG TATTTCAACTGTTC

LEFT PRIMER ATGGCGAACAAATACGAACA RIGHT PRIMER TGTAGGAGTAGCAGCAGTAC PRODUCT SIZE: 201

67. G1_Unigene_BMK.11522 p6 (ATGGCA)3 1810181035 GGCGAAGCGTCTTGGAGTCTCTGGAAGGTGCTTTTCG TACGAACCAAGAAGCCAAATCGATTCGAGAGAGGAGG CCGTTGCGCTCGAAGATCGACCAATCCAGCGCAG CTCGAAGCTCAAGAATGGTGATTGGAAATCGAAGG
CCGCGATCGAACGGAATGCTGCCGCTGCTGGCACTT CACGCCGTCAGCGAGTATTACAGGCTCGACCGG AAACCGCGCGTCACCGCCGGACTCATCGCCGCCAACG CTCTCGTCTACTTGCGCCCAGCTTTTCTCGACAA TATTCTTCCATCTATTCAAGAAGTCGgGTTCAA TCCTCACCTCATCCTCAAGTATGGGGACTTGAAGCGG TTCTTCTTGTCGCCGTTCTACCACGCCGGAGAG CCTCACTTGGTTTACAACATGCTCTCCCTGTTATGG AAGGGGATTCAGTTAGAATCTTCAATGGACAGTGCTG AATTTGCATCCATGGTTGCTGTCCTGCTGGGTATG TCCCAAGGAATGTCTCTGCTAATTTCCAAGTCCCTACT CATGTTCTTCGACTATGACCAGTCTTACTACCATG AATACTCTATTGGGTTTTCTGGGGTTCTCTTTGCCATG AAGGTTGTGCTCAATTCACAGACTGAGAACAGTTATG TGTATGGAATTCCAATACCGTCTCATTATGCTGCG TGGGCAGAATTAGCTCTTGCCCAAATGCTGGTACCTGG TGTCTCTTTTATTGGCCATTTGGGTGGAATACTTG CCGGTCTTGTCTATATCCGGCTGAGGGGTTCATA TAATGGTCCTGACCCACTTACTGCTATGTTCAGAGGTG TTTATGGTGTGCTGAAATGGCCTCTGCGGTTTTTACAG CGCTTGTTTCCGCGGAGGCAAGTATCCGGTAGAGG ACGTGTTGGTGGGGGTCAGAGAGGAATGTCTG TAACTGGTTTCTGGAGGTGCCATGCTTGTACGTATGAG AATTCTGCTTGGCTAGATGTATGTGAAATGTGTTCG ACAAATAGGAGAGGGGATGGCAATGGCAATGGCAG TGGCATTTCACCTCGATCACCACATTATTCCGGTG ACCTTACTTTGGAAGAGTTACGACGGCGGAGGTTGG AAAGATTTGGTAGATGGTAGGGTCCTAACTCATTAGG CTTGTTACAGATGCCAGGTGGTGTTGGCAATGCAGG A C A T G T A A A T T C A A A T A C C T C T T C T T T C C C TCTTCCCATTTCATTTGAATGTTTCAATCTGTCAT CATTGCTCGCACCCGATCTTATGCTGTTTTGTCGATC CATTTGATTAAAACTTTGAACATGTCTTGAGCAATCTG ATATTTGAGAATTCTATGGTCGGAGGATGGCCTGCTTT TATGTTTTGTGTATGAACATATATACTTCCAAAATTC TATAAATAGGAAGAGTTGAT

\section{LEFT PRIMER TGTAACTGGTTTCTGGAGGT} RIGHT PRIMER GGACCCTACCATCTACCAAA PRODUCT SIZE: 207

\section{G1_Unigene_BMK.11609 p6 (TTTTTA)3 18746763}

GATAAATTGACCATGCCGCACCGTTTCAATTGGGCG CAGAGAAGTAATCGGACCATACATCTCCAGATCT CAAAATCCCGTCATCGACCCACCCGCCATGGCCTC CTGGCCCGCCTCCTCCGATCGCTCTAATCAGGAGGGTG CTGCTGCAATCCGAAAGAGACAACCAGGACATCAAAT CAAGTTCTTGATTCCTCTTATATATGCCCCTGTTCT TCCCCTCATTCGACTCTCATTGCGGAGGAATCCGGTTG TGCGGGACCGCTTGTTTACTGCTGTGCTGGTTGGAGCG TTTGCTCATGGCTTTTATTTGGTGTATCCTTTTTTG TACTTAGTTTTTTATGGTGATTGAGAGATTATATATTG AATAGATAGATTGGTGCTATGAGAAATGACATGTTTTG ATTGATATGAGTTGCGGAATTGATGTGAATTGGTGG AAGAATTAGTAACTTAGGTTTGAAATCGGAACCCG TGGTAGATTTTCGCATTGATGCCAATATTTTGGATTTTT TAGTCCGAAATCTTGACAGCAAATGCGAAAGATGTG TGTTCGAATAAGATGGGGCTTGGTTTTGCTAACATTAG AGCATGTTCTGGTGGTATATGTAGGTGAAAAGGAG CAGGGTAGGGGAAATGGTTTTAGAGAATGTTCG ATTTGCAAGCTAGAATTTGTATTGTTGATACGGTG ATGGTTTGAGCCGAGCCCTCTTAGTGGGATAAGG 
CTTGGTGGTTGTTGTTGTTGTTTTTATTTTTATTTTTATG CGGTGATGGTTTGGAGATATGAACTGTTGAAATAG A T T G T C T T C GA GC T C CGT T CA T GT T G T T G T T G TTATTTTGATGTGGTGATGGTTTGGAGATATGAACCG TTGAAATAGATTAGCTTCTGGCTTCTTTGATACATGA

LEFT PRIMER TGAAAAGGAGCAGGGTAGGG RIGHT PRIMER ACAACAACATGAACGGAGCT PRODUCT SIZE: 217

\section{G1_Unigene_BMK.11759 p3 (GGA)7 2118521872}

GTAGACTTTCTCAGAAATTCTCATTTGGTTTCAGATG AGGATTTAAGACCAGAAACTCACTCCCCTGAAAAAG TACACAGTGAAAAAAGATTCCAAATCACAAAAGAGG CAGGACTTTATCCAAACAGAAAAGCCTTTGATTGTGG AGACATCTCACTTTTAACCATGTGCACCCAAAG AAAAAGACCCCTCCTTTTTAGGACAAAACCTG AACCCACCCCCAAATTTCAAATTCCTCCTCCATTTCTTC TGTTTTACATTGGGATGGGATAGTGACGTAACAGAGG CTCTGTCCTCATCATGAACATAAAAAACCACGTAAG TCTTAAACCCTAATTTTAGAAATCACAAACAGTACT CAGTATTGAAGAGTGAAAAGTCAAGATCTAGCTG TCTCTGTGTTGTAAACCACATTGTGCCCTCCTG TCATTTCTCCCCTCTTCATTTACTGTTGAGGATACCGG CATGTCCCGTAAGTTGGATCACTGAAAGTAACCAAG CCAGTGCCCTTGAAGTCACAATTCCAGTAGTTCCTG CCATGCATCTGATAGTAAGCATTCATAGCATACGATG CATGAGCAAGCAGCTTATCAGGCTCGAAACAAT CACCGTGCTCGTAAATCTCCCTGCAGTCCACAC CACCTGCTCCGCAGCAAAAATCCAACACAGCTTGAAG CACCTTATCATCGGCGTGTGGCTTAGCCACGCAC CAAACTGACGGTCCCCTCAACACTCCCGCTACTTTCT CACCAAATCCAGAGCCTCCTCCGGATCCACTTCCG TCAGTGTTGCTGCAGAATTGGCAGCTCAAATCCA CATTGTACACCTTCGATCCGTCCCCGTTGAAAATCC CAAAATTTCTCTCCGTCACGCCCCCTTCTTTTTTGTT CTCGTTGAACAAAGCAAACACAAATATTTCAACCTTG TCATTAGGCTTCATAGGCGTCCCTTTGTTAGACTGGG CACGCTCAATCAACCTCGTGTTATAAGTCTTGGCAT TATCTGGCGTAGCCGCCATATCTCCGGAGTCACCT TTGGACGGCCACCCGGATTCCGACACCATGATCTC CACCTTCCGGTGGCCAAGTCCCAGTGCATCAATGG CAGATCGAACAGCATCGATTTGTGCATCCAGCATG TTGGTGTATACATAGCCCTTGGGGTCACGCACGCTGG TTGAGGTTGAGTTCCCCAGTAATGCGTACTGTAGATTG ACCATGTCGGGGTTGTCCCGGTAGGCGAAATAAGGG TAGGCATTGACCATGAAAGGGGCGTTAGTGTCAG CCAAGAAGGCGGCTATAGAGGTCATAGTTGGCGCGAG ATTTGTAGTGAAAGTGGAAGCTGAAGGAGGAAACG AGGATGCCAAAACAGCCATGCTGTGCGGGGTGGTG ACCTTGATCTTGCGGTCCAGCCCTCGGGCTATAAG CACCGAGTGCAAGTTCTGCATTGCTTGGACCAGAG CTGCTGGGTCCGATGGGTTTGGGTCCTTCTCATCG TTTCTTGCGGCAGTAGTAGTTGTTAAGTACTCG TTTCCTACGGCAATGGCAGTGATGGAAGTGGCGGG AATGAATGGCAGGACGCGAGTGGCAAACCACTCGTC CGCGGCGGCCACT T C C GT GCT GA T GT T GGGG ACATGGCTGTTCTCCACAGCGACGATGAGGTCG ATCCCGGTGTTGGAAAAGGCCTGGAGGATTTCGGGG
TTGGTGTCGTAGATTTTGACCTTGTCTATGAGAGTGG CTTGGAGGAGCTGAGCAACTCTCTTTGGCGGTGG CAGGTTGTTTCCAAGAGTGCCGTAGTTTATGCCTAC AGCTGAAACAACGGCGTTCGGGAATGGGCTGAAAA TCAAAGGGTGGAGGAGGAGGAGGAGGAGGATGAG TGAGATAGGATGGAGGGGATATCAGGGGGAAGG AACTTGTCCCAAACAGCGTCGGAATCGGCGGCAG ACATGAAACTCTTAGACACCGCCGAAAACCTGCACG CGTCTCGTGGCGTCGTTAAGGAGACGACGTTAG CTATGCATCCTTCGGGCAACTCCTGCAGATCCATGTG AACGTGATGATGATCGCCATCGAGTACCATCG A C A C C G G C G T C C C G C A G C C T C C GCA G G T G ATTCCTCCTCCTCCTCCTCCTGATGCACCGGCGTCG ACTGTCTTCTTTTGCAACTTTGGTTGATCTCCG CCGCGGCGGGGTCTCATGACGTATTCTGTCTGTCTG TCTGTGTCGTTGGCTCTCGTCTCGTGCGTGTACTTTG TGGTACGTGTTCTGTTGAGCGAACGAAACTTGTCA TATTTGTATGGACTAGGGGGAGAGGCCTTACGCGTT AAGGCATGGTTCGATTTTTCCTCGAATTTTACTTTG TTTTCTAATGTTGACAAACGCTGCACTTTCT

\section{LEFT PRIMER GTTGTTTCCAAGAGTGCCGT} RIGHT PRIMER TCGGCGGTGTCTAAGAGTTT PRODUCT SIZE: 194

70. G1_Unigene_BMK.11787 p5 (CCGAA)4 20207226 GACATGTATTAATCGTCATGAATCCGACTCGCTATA TAAAAAGGTGGATACAAAATCCGTCGTAAATTAGG TGGGGGTAATCCGATAAAAAGCTCAGAAACAA TCAATCCGAATCGTCATCGAGTGCACGTTCCTTG T T C G A A C G A C C A T C T A A G A C A T C C A T C C A TATCCTTCGTCATCCTTTATCCGACCGACTGGATCCT CATCCGAACCGAACCGAACCGAAGTCGATTGTG ATCGAGGACGAGTTTAGATCTTGGTGTTGGTGTTGG AAGCACCGGTGGCACCTCCTCCGACTCGGGGAG CACCCTCGTAGAACTGGTGCTTGTGTCCGTTGTCGG A A TGGGCGTGGGGCCCCGAAGCCTGCTGCTG CTTCAAAAAGTCGGCGTGCGAGATGGTGGGTAAG ACGGTCGCCTCGTGGACGATGGGGGCGTCGTG AATTTTCTCGCTTACAGCTTGAGTATGGTGAATGG TTGTTGGCTGGACAGTCTCACGCTGGATAACAGG TTGGATGGTCTCATGAATGTGGTGGTGTTCGTG CTGGTTGACGTGTGTACCGAGGTGCGCACCGGTCCG CTCGACGTCGGTGGTGCTCTGGGTGTTCTGGTACG CCGA GCGT T GC T CGT GA A GC T T C T GCTGGT G CTCAGGAAGCATATCTTCTTTGTGTTCCCTGACTA CGGTAGGGGCGACCGAGTTGACATGCTTGGTCTCG AGGATCTTGTGGTCCTCGATAGGCTGGACCCGGTG TTGGTGGTGGTGAACGTGTCGCTCTCGGTCGATAG CCTCAGCGGTCTCGGTGTGTTCTTTGGGTCGGATAG TCTCCTGGGTGACCTCGGGTGCGACCTCGGAGCAG ACCTCGGTGGAGTCGGGCTTGTGCGAGCCCTTAAGG AAATCCTTGACGCCTTGGGTGATACCTGACATG CTTATTGATGGTTTTGGATTGgGTAAAGAGAGAG AAGAG

LEFT PRIMER TCGAACGACCATCTAAGACA RIGHT PRIMER GACACAAGCACCAGTTCTAC PRODUCT SIZE: 194 